quatrième série-tome 42 fascicule 1 janvier-février 2009

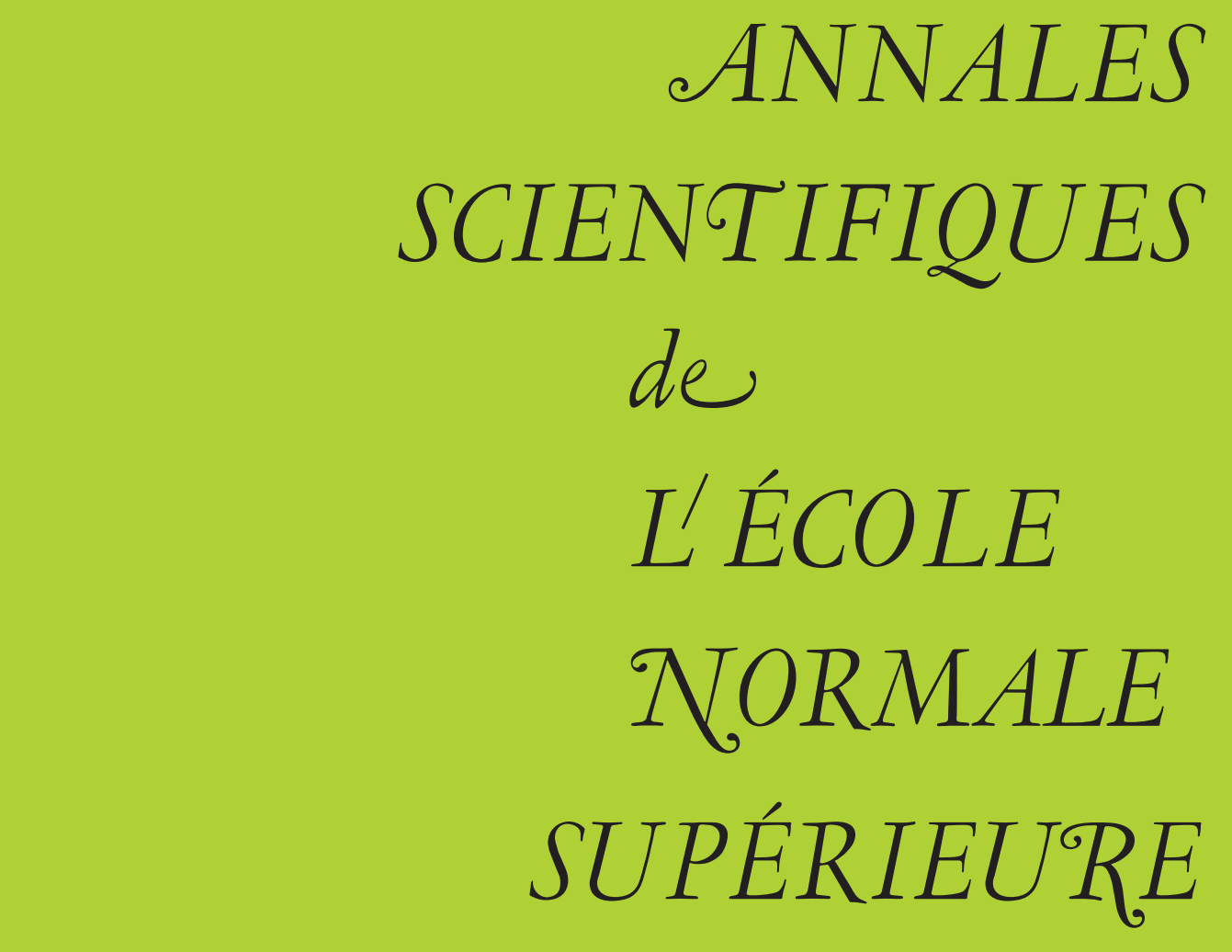

Thierry De PAUW

Size Minimizing Surfaces 
Ann. Scient. Éc. Norm. Sup.

$4^{\text {e }}$ série, t. 42, 2009, p. 37 à 101

\title{
SIZE MINIMIZING SURFACES
}

\author{
By Thierry De PAUW
}

Abstract. - We prove a new existence theorem pertaining to the Plateau problem in 3-dimensional Euclidean space. We compare the approach of E.R. Reifenberg with that of H. Federer and W.H. Fleming. A relevant technical step consists in showing that compact rectifiable surfaces are approximatable in Hausdorff measure and in Hausdorff distance by locally acyclic surfaces having the same boundary.

RÉSUMÉ. - Nous obtenons un nouveau théorème d'existence relatif au problème de Plateau dans l'espace euclidien de dimension 3. Ce faisant, nous comparons les approches d'E.R. Reifenberg d'une part, et de H. Federer et W.H. Fleming d'autre part. Un pas technique important consiste à démontrer qu'on peut approcher tout ensemble compact et rectifiable, en mesure de Hausdorff et en distance de Hausdorff, par une surface localement acyclique ayant le même bord.

\section{PART I \\ INTRODUCTION}

\section{Foreword}

The Plateau problem can be stated informally like this: Given a boundary $B \subseteq \mathbb{R}^{3}$, we seek a surface $S \subseteq \mathbb{R}^{3}$ spanning $B$ and having least area among all such surfaces. Solving the problem partly consists in making sense of the italicized words. One expects that the minimizing surfaces model soap films, which are the objects J. Plateau was interested in, [22]. In his classical book [3], R. Courant reports on the work of J. Douglas where surfaces are understood as continuous mappings. This setting is shown to be an actual restriction for instance in W.H. Fleming's paper [14].

The author is a chercheur qualifié of the Fonds National de la Recherche Scientifique, Belgium. 
We start by recalling why an application of the direct method of the calculus of variations may be a troublesome task. Indeed some minimizing sequence may have "thin tentacles", or "filigree", that will not contribute for a lot of area but yet might persist for some substantial part of the limit (e.g. in the sense of Hausdorff distance). Think of $B$ being a (planar) circle and let $S$ denote the 2-dimensional flat disk bounded by $B$. Referring to the observation that the nearest point projection on the plane containing $B$ and $S$ has Lipschitz constant 1 (and therefore does not increase area), we infer that $S$ is the unique area minimizer in any reasonable sense ${ }^{(1)}$. Choose, for $S_{j}, j=1,2, \ldots$, the flat disk $S$ from which $j$ nonoverlapping small disks have been removed and replaced with "curvy conical" surfaces (the tentacles) whose vertices are points $a_{1}, \ldots, a_{j}$ chosen in advance. This can be done in order for the total contribution in area of the tentacles to be bounded by $j^{-1}$, so that $S_{1}, S_{2}, \ldots$ is indeed a minimizing sequence. The reader may enjoy tickling their imagination by staring at Figures 1.3.1-1.3.4, in [20]. Letting $a_{1}, a_{2}, \ldots$ be a dense sequence in space we see that we can arrange for the Hausdorff limit of that particular minimizing sequence to be the whole space $\mathbb{R}^{3}$. Therefore the required semicontinuity of area does not hold. One way to circumvent the problem is to modify the minimizing sequence (cutting off the tentacles and patching the holes with controlled disks); another way consists in considering a weaker concept of convergence of surfaces so that the filigree disappear in the limit. We explain below the two points of view.

Two nearly simultaneous theories were published in 1960. One by E.R. Reifenberg [23], and the other by H. Federer and W.H. Fleming [12]. Both dealt with general dimensions and codimensions - and as a matter of fact this was one of their main striking features - yet in this paper we will purposely restrict ourselves to 2 -dimensional surfaces in $\mathbb{R}^{3}$. We now turn to giving a brief account of these contributions.

\section{The approach of E.R. Reifenberg}

Assume for the sake of illustration that $B \subseteq \mathbb{R}^{3}$ is a simple closed Jordan curve, and $S \supseteq B$ is a compact set. We say that $B$ bounds $S$ if the homomorphism $H_{1}(B ; G) \rightarrow H_{1}(S ; G)$ induced in homology by the inclusion map is trivial. Upon a moment of reflection it should be clear that this indeed says that $S$ fills the hole in $B$ (see the intriguing example [23, Appendix, Example 9] though). The definition also readily depends on $G$, a fixed "coefficients group". Furthermore, as we shall see soon enough the choice of a particular homology theory is not indifferent. In this setting area is understood as the 2-dimensional spherical measure $\mathscr{S}^{2}$ (see $[11,2.10 .2(2)]$ for a definition ${ }^{(2)}$ ). Letting $S_{1}, S_{2}, \ldots$ be any sequence of competitors (i.e. compact sets bounded by $B$ in the above sense) converging to some $S$ in Hausdorff distance we first want to make sure that the boundary condition is preserved in the limit. This will be the case if we consider Čech homology groups in the definition of " $B$ bounds $S$ " (see Proposition 17.1). After possibly projecting the sets $S_{j}, j=1,2, \ldots$, on the convex hull of $B$ we infer from the Blaschke selection

\footnotetext{
(1) In the present paper we consider only area induced by the Euclidean metric of $\mathbb{R}^{3}$.

(2) In case $S$ is $\left(\mathscr{H}^{2}, 2\right)$ rectifiable then $\mathscr{S}^{2}(S)=\mathscr{H}^{2}(S)$ where the latter is the 2-dimensional Hausdorff measure of $S,[11,3.2 .26]$.

$4^{\mathrm{e}}$ SÉRIE - TOME $42-2009-\mathrm{N}^{\mathrm{O}} 1$
} 
principle that some subsequence $S_{j(1)}, S_{j(2)}, \ldots$ converges in Hausdorff distance. Before referring to this principle E.R. Reifenberg performs a careful cutting and pasting surgery on the sets $S_{j}, j=1,2, \ldots$, in order that semicontinuity of area holds along the modified minimizing sequence $\tilde{S}_{j}, j=1,2, \ldots$ Checking that the sets $\tilde{S}_{j}$ verify the same boundary condition as $S_{j}$ turns out to rely on the Exactness Axiom of EilenbergSteenrod (among many other things of course). This axiom is verified when $G$ is a compact abelian group (see [8, Chap. IX, Theorem 7.6]) but not necessarily otherwise (in particular exactness does not hold when $G=\mathbb{Z}$, see [8, Chap. X, §4]). Thus existence theory in this setting is restricted to the case when $G$ is compact and abelian, and in fact E.R. Reifenberg concentrates on $G=\mathbb{Z}_{2}$ and $G$ the group of reals modulo 1 .

We are now ready to state a corollary of E.R. Reifenberg's work. Letting $B \subseteq \mathbb{R}^{3}$ be a closed simple Jordan curve and $G$ be a compact abelian group, the following variational problem admits a minimizer:

$$
\left(\mathscr{P}_{R, G, B}\right)\left\{\begin{array}{l}
\text { minimize } \mathscr{S}^{2}(S) \text { among compact sets } S \supseteq B \\
\text { such that } \check{H}_{1}\left(i_{B, S}\right): \check{H}_{1}(B ; G) \rightarrow \check{H}_{1}(S ; G) \text { is trivial }
\end{array}\right.
$$

where $i_{B, S}$ denotes the inclusion $B \rightarrow S$. Moreover Reifenberg proves that if $S^{*}$ is a (proper) minimizer of the problem then in a neighborhood of $\mathscr{S}^{2}$-almost every $x \in S^{*} \backslash B$, the set $S^{*}$ is a topological disk. In a subsequent analysis [24] he was able to improve this regularity result to showing that at such point $S^{*}$ is in fact a real analytic graph.

\section{The approach of H. Federer and W.H. Fleming}

Here boundaries and surfaces are meant as currents. An $m$-dimensional current in $\mathbb{R}^{3}$ is a continuous linear form on the space $\mathscr{D}^{m}\left(\mathbb{R}^{3}\right)$ of smooth differential forms of degree $m$ with compact support. A current $T \in \mathscr{D}_{m}\left(\mathbb{R}^{3}\right)$ is called rectifiable whenever the following holds. There exist

1. A bounded $\mathscr{H}^{m}$ measurable $\left(\mathscr{H}^{m}, m\right)$ rectifiable set $M \subseteq \mathbb{R}^{3}$;

2. An $\mathscr{H}^{m}$ measurable orientation $\xi: M \rightarrow \wedge_{m} \mathbb{R}^{3}$;

3. An $\mathscr{H}^{m}$ measurable multiplicity $\theta: M \rightarrow \mathbb{Z} \backslash\{0\}$;

such that

$$
\mathbf{M}(T):=\int_{M}|\theta| d \mathscr{H}^{m}<\infty
$$

and

$$
\langle T, \omega\rangle=\int_{M}\langle\omega, \xi\rangle \theta d \mathscr{H}^{m}
$$

whenever $\omega \in \mathscr{D}^{m}\left(\mathbb{R}^{3}\right)$. By $M$ being $\left(\mathscr{H}^{m}, m\right)$ rectifiable we mean that $\mathscr{H}^{m}(M)<\infty$ and there are finitely many or countably many $m$-dimensional submanifolds of class $C^{1}$, $M_{1}, M_{2}, \ldots$, such that $\mathscr{H}^{m}\left(M \backslash \cup_{j} M_{j}\right)=0$. This implies $M$ has an $m$-dimensional approximate tangent space $\operatorname{Tan}^{m}(M, x)$ at $\mathscr{H}^{m}$-almost every $x \in M$ (see e.g. [11, 3.2.16,3.2.19] or [26, 11.4,11.6]). At such points $x \in M$ an orientation $\xi(x)$ consists in a unit $m$ vector generating $\operatorname{Tan}^{m}(M, x)$. The integer multiplicity $\theta$ can be thought of as the number of sheets passing through a point. The combinatorics of "sheets" and "multiplicities" accounts for the way the boundary of $T$ is computed: The boundary operator $\partial$ of currents is defined by duality 


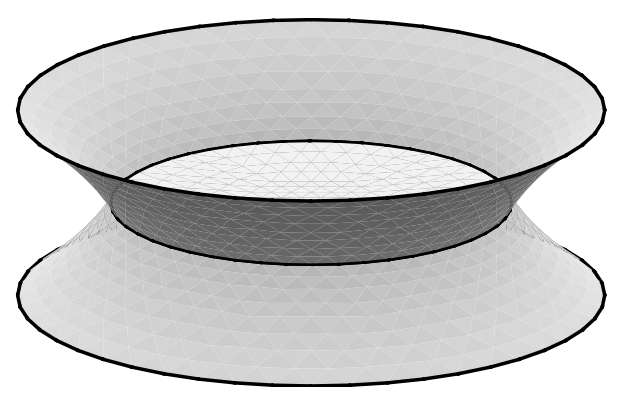

FIGURE 1. Size minimizing but not mass minimizing

of exterior differentiation, thereby generalizing Stokes' theorem for smooth orientable surfaces $M$. In this context the area of a 2-dimensional rectifiable current $T$ is understood as the mass $\mathbf{M}(T)$ defined in (1) — the $\mathscr{H}^{2}$ measure of the underlying set $M$ counting multiplicities.

The group of $m$-dimensional rectifiable currents in $\mathbb{R}^{3}$ is denoted by $\mathscr{R}_{m}\left(\mathbb{R}^{3}\right)$. We say $T \in \mathscr{R}_{m}\left(\mathbb{R}^{3}\right)$ is an integral current if also $\partial T \in \mathscr{R}_{m-1}\left(\mathbb{R}^{3}\right)^{(3)}$. The group of $m$-dimensional integral currents in $\mathbb{R}^{3}$ is denoted by $\mathbf{I}_{m}\left(\mathbb{R}^{3}\right)$. The compactness theorem relevant to the Plateau problem is the following.

3.1. TheOREM (Federer-Fleming). - Let $T_{1}, T_{2}, \ldots$ be a sequence of 2-dimensional integral currents in $\mathbb{R}^{3}$ whose supports are all contained in some fixed compact set, and such that $\sup _{j} \mathbf{M}\left(T_{j}\right)+\mathbf{M}\left(\partial T_{j}\right)<\infty$. There then exists a subsequence $T_{j(1)}, T_{j(2)}, \ldots$ converging weakly* to a 2-dimensional integral current $T$ in $\mathbb{R}^{3}$.

The weak* convergence to a current of some subsequence of $T_{1}, T_{2}, \ldots$ follows from the uniform mass bound together with the Banach-Alaoglu theorem and the separability of $\mathscr{D}^{2}\left(\mathbb{R}^{3}\right)$. Thus the deep content of the theorem is that the limit $T$ is rectifiable as well. We notice that the boundary operator $\partial$ is continuous with respect to weak* convergence and that mass is lower semicontinuous. The latter follows from the following formula:

$$
\mathbf{M}(T)=\sup \left\{\langle T, \omega\rangle: \omega \in \mathscr{D}^{m}\left(\mathbb{R}^{3}\right) \text { and }\|\omega(x)\| \leqslant 1 \text { for all } x \in \mathbb{R}^{3}\right\}
$$

where $\|\cdot\|$ is a suitable norm on $\wedge_{m} \mathbb{R}^{3}$. Thus the following variational problem admits a minimizer:

$$
\left(\mathscr{P}_{F F, \partial T_{0}}\right)\left\{\begin{array}{l}
\text { minimize } \mathbf{M}(T) \\
\text { among } T \in \mathscr{R}_{2}\left(\mathbb{R}^{3}\right) \text { with } \partial T=\partial T_{0} .
\end{array}\right.
$$

Here $T_{0} \in \mathscr{R}_{2}\left(\mathbb{R}^{3}\right)$ is fixed. The filigree disappear automatically in the weak* limit due to cancelations of orientations of nearby points in "horizontal sections of the tentacles".

\footnotetext{
(3) The condition is void when $m=0$.

$4^{\text {e }}$ SÉRIE - TOME $42-2009-\mathrm{N}^{\mathrm{o}} 1$
} 


\section{Mass versus size}

Mass minimizing currents model some but not all soap films. For instance if $\partial T_{0}$ consists of two similarly oriented circles lying in close parallel planes then the mass minimizer is the sum of the two oriented flat disks bounded by these circles rather than the singular surface shown on Figure 1. This is because in order to meet the boundary requirement, a current supported in that surface must have multiplicity 2 on the middle disk and hence has larger mass than the two multiplicity 1 disks as is implied by the triangular inequality. As a matter of fact if $T^{*} \in \mathscr{R}_{2}\left(\mathbb{R}^{3}\right)$ is a mass minimizer (a solution of $\left(\mathscr{P}_{F F, \partial T_{0}}\right)$ ) then $\operatorname{spt}\left(T^{*}\right) \backslash \operatorname{spt}\left(\partial T^{*}\right)$ is a real analytic submanifold of $\mathbb{R}^{3}$ according to a theorem of W.H. Fleming [15].

The surface shown in Figure 1 can be realized as a soap film. As a current it minimizes size rather than mass. The size of a rectifiable current $T \in \mathscr{R}_{2}\left(\mathbb{R}^{3}\right)$ is the area of the underlying rectifiable set without counting multiplicities, i.e.

$$
\mathbf{S}(T)=\mathscr{H}^{2}(M) .
$$

The Plateau problem can now be put as follows.

$$
\left(\mathscr{P}_{F F, \mathbf{S}, \partial T_{0}}\right)\left\{\begin{array}{l}
\text { minimize } \mathbf{S}(T) \\
\text { among } T \in \mathscr{R}_{2}\left(\mathbb{R}^{3}\right) \text { such that } \partial T=\partial T_{0}
\end{array}\right.
$$

Unfortunately a size minimizing sequence need not be bounded in mass (see the example given in [7, Introduction, p. 407-408]) so that the compactness theorem 3.1 does not apply. The paper [7] proves the existence of currents which minimize an energy interpolating between mass and size. In case $\partial T_{0}$ is a smooth submanifold contained in the boundary of a convex body then F. Morgan has proved existence of a size minimizer [19] (this result holds in general dimension and codimension 1, the proof shows that some size minimizing sequence is uniformly bounded in mass). However existence of a size minimizing current is unknown for instance when the given boundary curve $\partial T_{0}$ is a trefoil knot (see next section though).

Note that, akin to size, the energy being minimized by E.R. Reifenberg does not take the multiplicity into account. Since in the rectifiable currents setting one allows for integer multiplicities, it should now be clear that minimizing size among rectifiable currents corresponds to minimizing $\mathscr{S}^{2}$ in the Reifenberg context with coefficient group $G=\mathbb{Z}$. In the first case existence is unknown because of a lack of compactness. In the second case it is unknown because the suitable cut and paste procedure is not available to produce a bald minimizing sequence.

\section{Main result}

In the remainder of this paper $B$ denotes a smooth compact 1-dimensional submanifold of $\mathbb{R}^{3}$. We also let $B_{0} \in \mathscr{R}_{1}\left(\mathbb{R}^{3}\right)$ be a 1-dimensional rectifiable current such that spt $B_{0}=B$, i.e. $B_{0}$ consists in a choice of an orientation and multiplicity of each component of $B$. Furthermore we let $L$ denote the subgroup of $\check{H}_{1}(B ; \mathbb{Z})$ generated by $B_{0}$ (see Remark 17.4). Given a set $S \subseteq \mathbb{R}^{3}$ containing $B$ we say that the kernel of the homomorphism $\check{H}_{1}\left(i_{B, S}\right): \check{H}_{1}(B ; \mathbb{Z}) \rightarrow \check{H}_{1}(S ; \mathbb{Z})$ is the algebraic boundary of $S$. We will be interested in sets $S$ whose algebraic boundary contains $L$. 
We can then consider the following two formulations of the Plateau problem. First, in E.R. Reifenberg's context:

$$
\left(\mathscr{P}_{R, \mathbb{Z}, L}\right)\left\{\begin{array}{l}
\text { minimize } \mathscr{H}^{2}(S) \text { among compact }\left(\mathscr{H}^{2}, 2\right) \text { rectifiable sets } S \\
\text { containing } B \text { and whose algebraic boundary contains } L .
\end{array}\right.
$$

Second, in the setting introduced by H. Federer and W.H. Fleming:

$$
\left(\mathscr{P}_{F F, \mathbf{S}, B_{0}}\right)\left\{\begin{array}{l}
\text { minimize } \mathbf{S}(T) \\
\text { among } T \in \mathscr{R}_{2}\left(\mathbb{R}^{3}\right) \text { with } \partial T=B_{0} .
\end{array}\right.
$$

Our main result reads as follows.

5.1. THEOREM. - With the same notations as above one has:

(A) $\inf \left(\mathscr{P}_{R, \mathbb{Z}, L}\right)=\inf \left(\mathscr{P}_{F F, \mathbf{S}, B_{0}}\right)$;

(B) The variational problem $\left(\mathscr{P}_{R, \mathbb{Z}, L}\right)$ admits a minimizer.

Proving conclusion (A) amounts to constructing a minimizing sequence for one problem which is also a minimizing sequence for the other problem. Note this is not trivial since it is not clear whether a competitor for one problem is also a competitor for the other. In other words it is not immediately obvious that any set $S$ whose algebraic boundary contains $L$ supports a rectifiable current whose boundary is $B_{0}$, and vice versa. The question boils down to the comparison of the Čech homology group $\check{H}_{1}(S ; \mathbb{Z})$ with the homology group $\overline{\mathbf{H}}_{1}(S)$ corresponding to the chain complex of integral currents supported in $S$,

$$
\mathbf{I}_{3}(S) \stackrel{\partial}{\longrightarrow} \mathbf{I}_{2}(S) \stackrel{\partial}{\longrightarrow} \mathbf{I}_{1}(S) \stackrel{\partial}{\longrightarrow} \mathbf{I}_{0}(S) \longrightarrow \mathbb{Z}
$$

In the section 17 we will state a theorem to that effect which has been proved in [5]. In order that this theorem applies, the minimizing sequence $S_{1}, S_{2}, \ldots$ needs to be slightly modified first, so that each $S_{j}$ is replaced with some locally acyclic ${ }^{(4)} \tilde{S}_{j}$ of nearly the same area. The relevant approximation procedure is developed in Parts 2 and 3 of the present paper.

As a matter of fact it is so far unknown whether the Čech homology group $\check{H}_{1}\left(S^{*} ; \mathbb{Z}\right)$ and the integral currents homology group $\overline{\mathbf{H}}_{1}\left(S^{*}\right)$ coincide in case $S^{*}$ is a minimizer whose existence is stated in (B). According to Proposition 17.3 it is sufficient to prove that $S^{*}$ is locally acyclic at each of its points $x \in S^{*}$. That this holds true at interior points $x \in S^{*} \backslash B$ is a consequence of J. Taylor's regularity theorem [27]. At boundary points though the situation is more complex and full regularity has not yet been proved (there are ten conjectured pictures arising at boundary points according to G. Lawlor and F. Morgan [18], see also [20, Figure 13.9.3]) ${ }^{(5)}$.

The lack of known boundary regularity makes it impossible to apply F. Morgan's method in [19, Theorem 2.11] (see also [7, Remark 2.3.5]) to prove the existence part (B) of our result. Recall that the point is to find a minimizing sequence which does not grow tentacles, in order that Hausdorff measure be lower semicontinuous along that sequence. To achieve this goal we will apply the Federer-Fleming compactness theorem for integral currents and find

\footnotetext{
(4) With respect to integral currents homology.

(5) Potential fun includes the case when the size minimizing set is a subset of that shown in Figure 1, and the new smooth boundary curve wanders on different sheets near the singular circle, intersecting that circle (when it changes from one sheet to another) along a Cantor set of positive length.

$4^{\text {e }}$ SÉRIE - TOME $42-2009-\mathrm{N}^{\mathrm{o}} 1$
} 

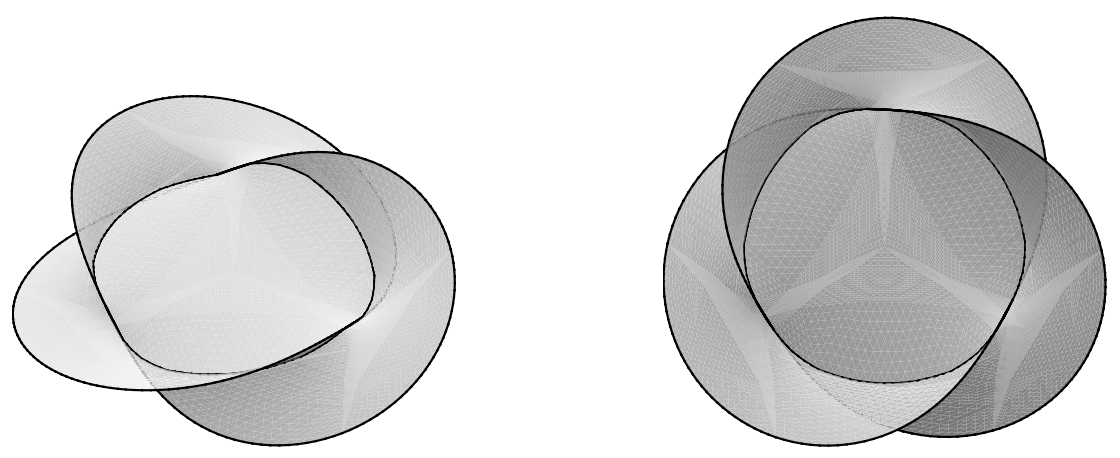

Figure 2. A conjectured size minimizer

a sequence $T_{1}, T_{2}, \ldots$ of currents which minimize size plus a small amount of mass (this penalization was studied in [7]). The key property that yields lower semicontinuity is the monotonicity of each such current. We face again the problem that $S_{j}=\operatorname{spt} T_{j}$ does not need to be a proper competitor in the setting of E.R. Reifenberg, yet the ad hoc approximation procedure evoked above fills the gap and we are able to produce the sought for minimizing sequence $\tilde{S}_{1}, \tilde{S}_{2}, \ldots$ without armful filigree.

We believe it is worth mentioning at least one example of a (conjectured) minimizer. Here we let $B$ be a smooth nicely symmetric trefoil knot in $\mathbb{R}^{3}$. We conjecture that the minimizing set $S^{*}$ of problem $\left(\mathscr{P}_{R, \mathbb{Z}, L}\right)$ is the one shown (from two different angles) in Figure 2. It is made of one "disk" in the middle to which three quarter moons ${ }^{(6)}$ are attached along portions of the given boundary $B$ and portions of a singular curve $C \subseteq S^{*} \backslash B$. Along the singular curve $C$ three sheets meet at equal angles of $120^{\circ}$. Some portions of the boundary knot $B$ touch one sheet of $S^{*}$, and some touch two sheets of $S^{*}$ (corresponding to the tangent cone number 5 in [20, Figure 13.9.3], and to the tangent cone number 4 in that list at "transition points" between $B$ and $C$ ). In order that the boundary condition be met one needs to give the middle disk a multiplicity 2 , whereas the quarter moons each have multiplicity 1 . I do not know of any technique to establish that the surface shown in Figure 2 is indeed a minimizer — calibrations apply mainly to proving mass minimization.

\section{An approximation theorem}

As we alluded to above, we shall need a specific approximation theorem for 2-dimensional "surfaces" in $\mathbb{R}^{3}$. The sets $X \subseteq \mathbb{R}^{3}$ that we wish to approximate are assumed to be compact and $\left(\mathscr{H}^{2}, 2\right)$ rectifiable. The latter is equivalent to saying that $\mathscr{H}^{2}(X)<\infty$ and at $\mathscr{H}^{2}$ almost every $x \in X$, the 2-dimensional Hausdorff measure carried by the homothetic expansions $\frac{X-x}{r}$ converges weakly as Radon measure (when $r \downarrow 0$ ) to the 2-dimensional Hausdorff measure carried by some linear subspace $W_{x} \in \mathbf{G}(3,2)$.

\footnotetext{
(6) Manufactured with soap of course! With this regard one can read the advices of J. Plateau reported by E. Lamarle in [17, Deuxième partie, $\$ 25$, second footnote] about mixing Marseille soap with French glycerol from Lamoureux. Alternatively one can use nowadays K. Brakke's surface evolver [2] to experiment with (virtual) soap films. The figures of the present paper were designed with that software.
} 
The approximating set $Y$ should be close to $X$ in Hausdorff distance and should have $\mathscr{H}^{2}$ measure close to that of $X$. Moreover $Y$ should be $\left(\mathscr{H}^{2}, 2\right)$ rectifiable as well. So far of course we could let $Y=X$, but most importantly we want $Y$ to be "simpler" than $X$ from a topological point of view - say that in small neighborhoods of each of its points $Y$ should consist in one piece with no hole nor handle and no little sphere floating around. Polyhedral sets constitute a good class of approximating sets to look for. Being finite unions of 2-dimensional parallelograms in $\mathbb{R}^{3}$ they certainly enjoy the required topological property. From the very construction of the set $Y$ it will turn out that there is a (Lipschitzian $\left.{ }^{(7)}\right)$ map $f$ defined in a neighborhood of $X$ such that $f(X) \subseteq Y$. Theorems of this flavor have by now become a classic in geometric measure theory, as for instance in the realm of integral currents [11, 12] (specifically the "Approximation theorem" [11, 4.2.20]).

The extra requirement ${ }^{\left({ }^{8}\right)}$ we want the approximating set $Y$ to meet is "to have the same boundary" as the original set $X$. Since $X$ is not assumed to be a current in the first place, we need to say what we mean by boundary of $X$. For the purpose of applying our result to the proof of our main theorem 5.1 we choose J.F. Adams' definition in the Appendix of E.R. Reifenberg's paper [23]. To make things simpler without missing the main point, assume that the given "boundary" $B \subseteq \mathbb{R}^{3}$ is a smooth simple closed Jordan curve in $\mathbb{R}^{3}$. We recall from the previous sections that $B$ bounds $X \supseteq B$ if the homomorphism induced by inclusion in homology $\check{H}_{1}(B ; \mathbb{Z}) \rightarrow \check{H}_{1}(X ; \mathbb{Z})$ is trivial ${ }^{(9)}$. Upon a moment of reflection it should be clear that this indeed means that $X$ fills the hole in $B^{(10)}$. With this vocabulary at hand we can state our added condition: in case $B$ bounds $X$ we want to find an approximating $Y$ which is bounded by $B$ as well. This will certainly hold if we can guarantee that $f$ mapping $X$ in $Y$ is so that $f(B)=B$ and the restriction $f \uparrow B$ is homotopic to the identity of $B$.

On the other hand it is now obvious that we should abandon the idea of approximating $X$ with a polyhedral set - think for instance of $B$ being a circle and $X$ being a spherical cap. We thus need to involve another notion of local topological connectedness. In order that it be applicable to proving our existence theorem 5.1 we use the chain complex of integral currents to compute homology groups in this context. Specifically if $Z \subseteq \mathbb{R}^{3}$ we consider the chain complex

$$
\mathbf{I}_{3}(Z) \stackrel{\partial}{\longrightarrow} \mathbf{I}_{2}(Z) \stackrel{\partial}{\longrightarrow} \mathbf{I}_{1}(Z) \stackrel{\partial}{\longrightarrow} \mathbf{I}_{0}(Z) \stackrel{\alpha}{\longrightarrow} \mathbb{Z}
$$

and the corresponding (reduced) homology groups $\overline{\mathbf{H}}_{q}(Z)$. Here $\mathbf{I}_{q}(Z)$ is the group of $q$ dimensional integral currents whose support is contained in $Z, \partial$ denotes the boundary operator of currents and $\alpha$ is the augmentation map defined as $\alpha(T)=\langle T, \mathbb{1}\rangle$ where $\mathbb{1}$ stands for any test function equal to 1 in a neighborhood of $\operatorname{spt} T$. We can now state the topological requirement we set for $Y$. Given $y \in Y$ and an open neighborhood $U$ of $y$ there should exist a smaller neighborhood $U^{\prime}$ of $y$ such that the homomorphism induced in homology by inclusion $\overline{\mathbf{H}}_{q}\left(Y \cap U^{\prime}\right) \rightarrow \overline{\mathbf{H}}_{q}(Y \cap U)$ is trivial for $q=0,1,2$. In that case we say that $Y$ is $(\overline{\mathbf{H}}, 1)$ locally connected. The homology theory evoked above is studied for instance in [5]

\footnotetext{
(7) The connoisseur will spot the need for $X$ being closed at this stage — this will be touched upon in section 7 .

(8) With respect to H. Federer and W.H. Fleming's approximation theorem.

(9) The use of Cech homology is by no means essential in the present introduction and the reader may safely assume this is singular homology (in hopes that this makes them feel better).

(10) Not quite though! See the intriguing example [23, Appendix, Example 9].

$4^{\mathrm{e}}$ SÉRIE - TOME $42-2009-\mathrm{N}^{\mathrm{o}} 1$
} 
where one shows the relevance of the stated local connectedness condition for comparison of $\overline{\mathbf{H}}_{1}(Y)$ with $\check{H}_{1}(Y ; \mathbb{Z})$.

We are now ready to state our approximation result.

\subsection{Theorem. - Assume that}

(A) $X \subseteq \mathbb{R}^{3}$ is compact and $\left(\mathscr{H}^{2}, 2\right)$ rectifiable;

(B) $B \subseteq X$ is either empty or a compact 1-dimensional submanifold of $\mathbb{R}^{3}$ of class $C^{3}$ (without boundary);

(C) $\varepsilon>0$.

There then exist a Lipschitzian map $f: \mathbb{R}^{3} \rightarrow \mathbb{R}^{3}$ and an $\left(\mathscr{H}^{2}, 2\right)$ rectifiable set $Y \subseteq \mathbb{R}^{3}$ verifying the following properties:

(D) $f(X) \subseteq Y$;

(E) $\operatorname{dist}_{\mathscr{H}}(X, Y)<\varepsilon$;

(F) $\left|\mathscr{H}^{2}(X)-\mathscr{H}^{2}(Y)\right|<\varepsilon$;

(G) $\mathscr{H}^{2}(\operatorname{Clos}(Y) \backslash Y)=0$;

(H) $Y$ is $(\overline{\mathbf{H}}, 1)$ locally connected;

(I) In case $B$ is nonempty, $f(B)=B$ and $f \uparrow B$ is homotopic in the Lipschitzian category to $\operatorname{id}_{B}$.

It is most likely that this theorem generalizes to any dimension and codimension even though some steps of the proof described below do not apply trivially to the more general situation. Investigations in this direction are under way, particularly so regarding the case when there is no added boundary condition, [13, 21]. Part 2 of the present paper deals with the no-boundary problem in any dimension but is restricted to codimension 1 . Part 3 deals with the presence of a boundary and is written entirely in the context of $\mathbb{R}^{3}$ for the purpose of homogeneity - the reader will notice which statements do not depend on the low dimension. At any rate the application to minimizing surfaces in theorem 5.1 is already new in 3dimensional space.

\section{Sketch of proof of the approximation theorem}

We now turn to roughly describing the proof of Theorem 6.1 with no attempt at rigor ${ }^{(11)}$. We start by explaining how to construct an approximating set $P$ dismissing for now conclusion (I) of our theorem, i.e. assuming there is no boundary condition to be preserved (this is the content of Theorem 11.4). Let $G$ denote the subset of $X$ consisting of those points $x$ such that (the 2-dimensional Hausdorff measure carried by) the homothetic expansions $\frac{X-x}{r}$ converge as $r \downarrow 0$ (in the sense of Radon measures) to (the 2 dimensional Hausdorff measure carried by) some plane $W_{x} \in \mathbf{G}(3,2)$. If $C_{x, r}$ denotes a cube centered at $x$ of diameter $r>0$ and $S_{x, r}=X \cap C_{x, r} \backslash \mathbf{B}\left(x+W_{x}, \eta r\right)$ is the part of $X$ in $C_{x, r}$ which is not $\eta r$ close to $x+W_{x}$ then the weak convergence as Radon measures guarantees that $\mathscr{H}^{2}\left(S_{x, r}\right)<\eta r^{2}$ for $r>0$ small enough (depending on $\eta$ and $x$ ) and $x \in G$. The set $X$ being rectifiable we recall that

(11) In order to avoid confusion we denote by $\eta>0$ a small real number whose role will be to control local error terms, and it should be thought of as being smaller than $\varepsilon$ assigned in the statement of the theorem. 
$\mathscr{H}^{2}(X \backslash G)=0$. Therefore (referring to Besicovitch's covering theorem) we can find pairwise disjoint finitely many cubes $C_{x_{1}, r_{1}}, \ldots, C_{x_{p}, r_{p}}$ centered at $G$, whose diameter is smaller than some $r_{0}>0$ to be determined later, and such that $\mathscr{H}^{2}\left(X \backslash \cup_{i=1}^{p} C_{x_{i}, r_{i}}\right)<\eta$. In order to keep the notation short we put $C_{i}=C_{x_{i}, r_{i}}$. We now explain what is the intersection of the (sought for) approximating set $P$ with each cube $C_{i}$. The main piece of $P$ in $C_{i}$ is the flat piece $\left(x_{i}+W_{x_{i}}\right) \cap C_{i}$ (having nearly the same area as $\left.X \cap C_{i}\right)$. We also add to $P$ the narrow strips in $\mathbf{B}\left(x_{i}+W_{x_{i}}, \eta r_{i}\right) \cap \operatorname{Bdry} C_{i}$ (which do not contribute much to area). The reason for adding these strips is that we want to define a map $f_{i}$ on $C_{i}$ such that $f_{i}\left(X \cap C_{i}\right) \subseteq P \cap C_{i}$, and the $f_{i}$ will be as follows. We divide $C_{i}$ into two convex bodies $C_{i}^{ \pm}$along the affine plane $x_{i}+W_{x_{i}}$. We will somehow choose points $b_{i}^{ \pm} \in C_{i}^{ \pm} \backslash \mathbf{B}\left(x_{i}+W_{x_{i}}, \eta r_{i}\right)$ and let $f_{i}^{ \pm}$denote the central projection on the faces of Bdry $C_{i}^{ \pm}$centered at $b_{i}^{ \pm}$. Since $b_{i}^{ \pm} \notin \mathbf{B}\left(x_{i}+W_{x_{i}}, \eta r_{i}\right)$ it is clear that the image by $f_{i}^{ \pm}$of the part of $X \cap C_{i}$ contained in the slab $\mathbf{B}\left(x_{i}+W_{x_{i}}, \eta r_{i}\right)$ is a subset of the part of $P$ defined so far. It remains to take care of the "dust" $S_{x_{i}, r_{i}}$. Remember that it has small area (relative to that of $X \cap C_{i}$ ). Now we need to choose properly the points $b_{i}^{ \pm}$so that the area of $f_{i}^{ \pm}\left(S_{x_{i}, r_{i}}\right)$ is not more than a fixed factor times that of $S_{x_{i}, r_{i}}$. That this be possible follows from the fact that the convex bodies $C_{i}^{ \pm}$are not too long and thin (thereby allowing for a control of the Lipschitz constant of $f_{i}^{ \pm}$away from $b_{i}^{ \pm}$, see Corollary 9.5) together with an averaging argument involving Fubini's theorem (see Proposition 9.7). We then add to $P$ some "polyhedral covering" of $f_{i}^{ \pm}\left(S_{x_{i}, r_{i}}\right)$. We also point out that by choosing $b_{i}^{ \pm} \notin X$, and referring to the compactness of $X$, the map $f_{i}^{ \pm}$is defined in a neighborhood of $X$ in $C_{i}$ (namely $C_{i}$ minus two tiny balls centered at $b_{i}^{ \pm}$) where it is Lipschitzian and homotopic to the identity.

Next we need to say what to do outside of $\cup_{i=1}^{p} C_{i}$. For that purpose we first observe that there is no major trouble in making the above argument valid if we replace the $C_{i}$ by nearby "pseudo-dyadic" sets, i.e. if we assume that the $C_{i}$ contain all the dyadic cubes that they intersect nontrivially (then the $C_{i}$ need not be exactly centered at $x_{i}$ anymore but it does not matter). Now the complement of $\cup_{i=1}^{p} C_{i}$ can be partitioned by small dyadic cubes, all of the same generation. Only finitely many of them, say $D_{1}, \ldots, D_{q}$, meet $X$. Again in each of these cubes we can define a central projection (centered away from $X$ ) pushing the part of $X$ in $D_{j}$ onto a set having area controlled by that of $X \cap D_{j}$. It should then be clear how to define $P$ in $D_{j}$ as well. The mapping $f$ defined in a neighborhood of $X$ is obtained by gluing together the pieces defined in the $C_{i}^{ \pm}$and in the $D_{j}$. That the global map be Lipschitzian follows from the fact that each local map coincides with the identity on the boundaries of the $C_{i}^{ \pm}$and $D_{j}$.

Looking at this construction we see that the Hausdorff distance between $P$ and $X$ is essentially controlled by the maximal diameter of the $C_{i}$ and the $D_{j}$; and that the Hausdorff measure of $P$ is essentially $\sum_{i=1}^{p} \mathscr{H}^{2}\left(X \cap C_{i}\right)$ and all the error terms are controlled by some multiple of $\eta$. We also see that we have defined a Lipschitzian map $f$ in a neighborhood $U$ of $X$, homotopic to the identity of $U$. From now on $f$ will be denoted by $f_{0}$.

Next we assume that $X$ bounds some smooth simple closed curve $B$. Of course $B$ may be moving under $f_{0}$, thus there is no reason that $B$ bounds $P$. We need to bring $B$ back to its original position. Notice that $B$ has not moved very far away, in particular it is contained in a tubular neighborhood $\mathbf{B}(B, r)$ of $B$ provided $\left\|f_{0}-\mathrm{id}_{U}\right\|_{\infty}<r$. We may choose $r>0$ so small that the nearest point projection $\pi$ onto $B$ is well defined in $\mathbf{B}(B, 2 r)$. Then we let $g_{r}$ : $\mathbb{R}^{3} \rightarrow \mathbb{R}^{3}$ coincide with $\pi$ in $\mathbf{B}(B, r)$, coincide with the identity outside of $\mathbf{B}(B, 2 r)$, and be 
an affine interpolation between both in the annular region (the "speed" of the interpolation along rays will matter below). We now discuss whether $f=g_{r} \circ f_{0}$ and $Y=g_{r}(P)$ verify the conclusions of Theorem 6.1. The fact that $f(B)=B$ and $f\lceil B$ is homotopic to the identity of $B$ are simple consequences of the choice of $r>0$ and the construction of $P$ applied so that $\left\|f_{0}-\mathrm{id}_{U}\right\|_{\infty}<r$.

We need to check the measure estimate appearing in conclusion (F), mainly that $Y$ has not much more area than $X$, in other words that the stretching of $P$ by $g_{r}$ near $B$ does not contribute much to area. The relevant error term here is $\mathscr{H}^{2}\left(g_{r}(P \cap \mathbf{B}(B, 2 r))\right)$. For one thing the Lipschitz constant of $g_{r}$ can be controlled independently of $r>0$ (but, of course, involves a dependence on $B$ ). This owes to a particular choice of an interpolation in the definition of $g_{r}$, see Theorem 12.3(B). Next we need to control $\mathscr{H}^{2}(P \cap \mathbf{B}(B, 2 r))$. Now the part of $P$ lying in the tubular neighborhood $\mathbf{B}(B, 2 r)$ comes from points of $X$ which are not more than a distance $\delta=\left\|f_{0}-\operatorname{id}_{U}\right\|_{\infty}$ away from that neighborhood. Here we need to know that $\mathscr{H}^{2}(P \cap \mathbf{B}(B, 2 r))$ is not more than $\mathscr{H}^{2}(X \cap \mathbf{B}(X, 2 r+\delta))$ (this is enough because the latter is small, provided $2 r+\delta$ is small, since $\mathscr{H}^{2}(B)=0$ ). In other words, we need a localized version of the measure estimate in the construction of $P$. Something like this can be obtained provided $r$ is essentially the same scale as $\delta$ (the scale of the cubes $C_{i}$ above, the maximal displacement performed by $f_{0}$ ). All of this can be taken care of, as stated in Theorem 11.4(G) (see also the beginning of the proof of the main result in section 16).

The main technical trouble that needs to be addressed is whether or not $Y=g_{r}(P)$ is $(\overline{\mathbf{H}}, 1)$ locally connected. Let $y \in Y \backslash B$. It is not hard to show (essentially Theorem 12.3(C)) that $g_{r}^{-1}\{y\}=\{x\}$ is a singleton and that $g_{r}$ is a $C^{1}$ diffeomorphism in a neighborhood of $x$. Since $P$ is $(\overline{\mathbf{H}}, 1)$ locally connected at $x$ (in fact a neighborhood of $x$ in $P$ is Lipschitz contractible in $P$ to $\{x\}$ ), so is $Y$ at $y$. The situation is different when $y \in B$. For instance it can occur that $\left(g_{r}\lceil P)^{-1}\{y\}\right.$ is an infinite set. Let us describe one way this can happen. Notice that the level sets of $g_{r}$ are flat disks (i.e. contained in affine planes; Theorem 12.1(D) and Remark 12.2(4)). From now on we denote by $W(y) \in \mathbf{G}(3,2)$ the plane such that $g_{r}^{-1}\{y\}$ is contained in $y+W(y), y \in B$. If also we denote by $\mathscr{W}_{P}$ the collection of 2-dimensional directions "used" by $P$, we see that $g_{r}^{-1}\{y\}$ is infinite when $W(y) \in \mathscr{W}_{P}$. This situation can be avoided by a small generic rotation of $P$, Lemma 13.4. Under some further mild conditions that are met generically as well it is possible to show that $g_{r}^{-1}\{y\}$ is finite (see the first half of the proof of Theorem 14.3, relying on Theorem 13.3 and Lemma 13.6). Now we look at $\left\{x_{1}, \ldots, x_{\kappa}\right\}=g_{r}^{-1}\{y\}$. Notice that each $x_{k}$ belongs to the "cylindrical" surface $M_{r}=\mathbb{R}^{3} \cap\{\xi: \operatorname{dist}(\xi, B)=r\}$. It is possible that $x_{k}$ belongs to several of the affine parallelograms ${ }^{(12)}$ that $P$ is made of. Let us denote by $\mathscr{W}_{P}\left(x_{k}\right)$ the collection of $W \in \mathbf{G}(3,2)$ such that $x_{k}+W$ "contributes" to $P$ (e.g. $\mathscr{H}^{2}\left(P \cap\left(x_{k}+W\right)\right)>0$ ). We want that for each $W \in \mathscr{W}_{P}\left(x_{k}\right)$ the intersection $\left(x_{k}+W\right) \cap M_{r}$ is a smooth curve (in a neighborhood of $x_{k}$ ). This will be the case provided $W \neq T_{x_{k}} M_{r}$. Translating $P$ by a small generic amount if necessary, we can assume this is indeed the case (Theorem 13.3(2)). Now we consider the small "half disks" $E_{k, W}=\left(x_{k}+W\right) \cap \mathbf{B}\left(x_{k}, \rho\right) \backslash \mathbf{U}(B, r), \rho>0$ small. Their "diameter" is a $C^{1}$ curve which gets closer to an actual diameter as $\rho$ gets close to 0 . Then a neighborhood of

(12) Actually $P$ will not be made of finitely many parallelograms, see Definition 11.1 , but this does not matter in our sketch of proof. 
$y$ in $Y$ is made of the union of the finitely many $g_{r}\left(E_{k, W}\right), k=1, \ldots, \kappa, W \in \mathscr{W}_{P}\left(x_{k}\right)$ (this requires a justification which will follow from stronger estimates that will be needed soon anyway). In order to show that it is acyclic ${ }^{(13)}$ we first look at each of the individual pieces. Of course a single $E_{k, W}$ is acyclic for there is a Lipschitzian deformation retraction in $E_{k, W}$ to $\left\{x_{k}\right\}$. We wish to copy one such deformation retraction from $E_{k, W}$ to $g_{r}\left(E_{k, W}\right)$, thereby showing that $g_{r}\left(E_{k, W}\right)$ is acyclic as well. This requires $g_{r} \uparrow E_{k, W}$ to be a bi-Lipschitzian homeomorphism. That this be the case will depend on the relative position of the three planes $W, T_{x_{k}} M_{r}$ and $W(y)$, namely their intersection should be $\{0\}$ - see Theorem 12.3(D). Some bad cases can be avoided again by a generic position argument, but not all of them - see Remark 12.4(3). Instead, in some cases we are forced to "pleat" $P$ near $x_{k}$ in order to force the directions in $\mathscr{W}_{P}\left(x_{k}\right)$ to meet the desired requirements. Such pleating must be performed so as not to increase much the Hausdorff area, see Lemma 14.1. Once this is done we end up with acyclic pieces $g_{r}\left(E_{k, W}\right)$. In order to show that their union is acyclic as well we would need some sort of Mayer-Vietoris argument which is not immediately available. Instead we want to prove acyclicity "by hand" (Theorem 15.1) using particular deformation retractions $H_{k, W}$ of $g_{r}\left(E_{k, W}\right)$ to $\{y\}$. We will use these homotopies $H_{k, W}$ to show that integer multiplicity rectifiable cycles supported in the union of $g_{r}\left(E_{k, W}\right), k=1, \ldots, \kappa, W \in \mathscr{W}_{P}\left(x_{k}\right)$, are boundaries of integer multiplicity rectifiable currents supported in the same set. The tools used here are the homotopy formula for currents [11, 4.1.9] or [25, §14], the explicit representation of 1-dimensional rectifiable cycles [11, 4.2.25] and the constancy Theorem [11, 4.1.31(2)]. In order for this to work we need the $H_{k, W}$ to meet consistency requirements. For instance we want that any two such maps $H_{k, W}$ and $H_{k^{\prime}, W^{\prime}}$ coincide on the common portion of boundary curve $B \cap E_{k^{\prime}, W^{\prime}} \cap E_{k, W}$. Notice that if $k$ is such that there are distinct $W_{1}, W_{2} \in \mathscr{W}_{P}\left(x_{k}\right)$ then there is also an intersection curve $g_{r}\left(E_{k, W_{1}}\right) \cap g_{r}\left(E_{k, W_{2}}\right) \backslash B$ along which we want $H_{k, W_{1}}$ and $H_{k, W_{2}}$ to coincide as well. For that purpose we consider deformation retractions on $E_{k, W_{1}}$ and $E_{k, W_{2}}$ which coincide on $E_{k, W_{1}} \cap E_{k, W_{2}}$. Here it is handy to assume that card $\mathscr{W}_{P}\left(x_{k}\right) \leqslant 2$ for each $k=1, \ldots, \kappa$. By genericity this is no restriction (Theorem 13.3(1)) provided we constructed our initial set $P$ with some care (see Definition 11.2). Having constructed the homotopies $H_{k, W}$ so that they verify the consistency conditions we can now glue them together and obtain a global continuous deformation retraction of the relevant neighborhood of $y$ in $Y$ on $\{y\}$. Therefore we conclude at once that this neighborhood is acyclic in singular homology. The acyclicity with respect to the homology of integral currents requires the extra argument sketched above, Theorem 15.1, which may be of independent interest.

Parts 2 and 3 of this paper consist in a documented version of the argument sketched above.

\section{Notations and preliminaries}

We use the following convention after D.H. Fremlin. The proof of a claim (which is itself part of the proof of a lemma, say) is given between the symbols $\mathbb{P} \ldots$...

(13) By $Z$ being acyclic here we mean that $\overline{\mathbf{H}}_{q}(Z)=\{0\}$ for $q=0,1,2$.

$4^{\text {e }}$ SÉRIE - TOME $42-2009-\mathrm{N}^{\mathrm{o}} 1$ 
Most of our notations are consistent with those of H. Federer's treatise [11] (see pages 670-671 op. cit. for a table of symbols), and the variants are self-explanatory. We start by a short review of the most useful symbols. We denote by Int, Clos and Bdry the topological interior, closure and boundary respectively. We let $\langle u, v\rangle$ be the canonical inner product of $u, v \in \mathbb{R}^{n}$. The associated Euclidean norm is denoted by $|\cdot|$, and sometimes by $|\cdot|_{2}$ when the companion maximum norm $|\cdot|_{\infty}$ is used in neighboring calculations. We define $\operatorname{dist}(x, A)=$ $\inf \{|x-a|: a \in A\}$ and $\mathbf{B}(A, r)=\mathbb{R}^{n} \cap\{x: \operatorname{dist}(x, A) \leqslant r\}$ and $\mathbf{U}(A, r)=\mathbb{R}^{n} \cap\{x:$ $\operatorname{dist}(x, A)<r\}$. When $A=\{a\}$ is a singleton we abbreviate $\mathbf{B}(a, r)$ and $\mathbf{U}(a, r)$. When the distance is defined in terms of the maximum norm instead, the corresponding balls are denoted by $\mathbf{B}_{\infty}(a, r)$ and $\mathbf{U}_{\infty}(a, r)$. The Hausdorff distance of $A, B \subseteq \mathbb{R}^{n}$ is dist $\mathscr{H}(A, B)$. The $m$-dimensional Hausdorff measure in Euclidean space $\mathbb{R}^{n}, 0 \leqslant m \leqslant n$, is denoted by $\mathscr{H}^{m}$. The Grassmannian $\mathbf{G}(n, m)$ is the collection of $m$-dimensional linear subspaces of $\mathbb{R}^{n}$. Finally, given sets $A \subseteq X$ and a function $f: X \rightarrow \mathbb{R}$ we let $f\lceil A$ denote the restriction of $f$ to $A$. The indicatrix function of $A$ is $\mathbb{1}_{A}$.

We now define some concepts for further reference and prove some elementary lemmas.

\subsection{Definition. - A set $E \subseteq \mathbb{R}^{n}$ is called $\mathscr{H}^{m}$ essential if $\mathscr{H}^{m}((\mathrm{Clos} E) \backslash E)=0$.}

Notice that an $\mathscr{H}^{m}$ essential set is also $\mathscr{H}^{m}$ measurable, and the finite union of $\mathscr{H}^{m}$ essential sets is $\mathscr{H}^{m}$ essential as well.

8.2. Definition. - Let $Y \subseteq \mathbb{R}^{n}$ and $m \geqslant 0$ an integer. We say that $Y$ is $(\overline{\mathbf{H}}, m)$ locally connected at $y \in Y$ if the following condition holds. For every open neighborhood $U$ of $y$ there exists an open neighborhood $U^{\prime} \subseteq U$ of $y$ such that the homomorphisms induced in (reduced) homology

$$
\overline{\mathbf{H}}_{q}\left(Y \cap U^{\prime}\right) \rightarrow \overline{\mathbf{H}}_{q}(Y \cap U)
$$

are trivial, $q=0, \ldots, m+1$. We say that $Y$ is $(\overline{\mathbf{H}}, m)$ locally connected if it is $(\overline{\mathbf{H}}, m)$ locally connected at each $y \in Y$.

In other words we require that for every $T \in \mathbf{I}_{q}\left(\mathbb{R}^{n}\right)$ with spt $T \subseteq Y \cap U^{\prime}$ and $\partial T=0$ there exists $S \in \mathbf{I}_{q+1}\left(\mathbb{R}^{n}\right)$ such that spt $S \subseteq Y \cap U$ and $\partial S=T$.

8.3. Example. - Assume that $y \in Y$ has a base of open neighborhoods each of which being contractible to $\{y\}$ in the Lipschitzian category, i.e. for every open set $U$ containing $y$ there exist an open set $U^{\prime} \subseteq U$ containing $y$ and a map $H:[0,1] \times Y \cap U^{\prime} \rightarrow Y \cap U^{\prime}$ with Lip $H<\infty, H(0, \zeta)=y$ and $H(1, \zeta)=\zeta$ for every $\zeta \in Y \cap U^{\prime}$. Then $\overline{\mathbf{H}}_{q}\left(Y \cap U^{\prime}\right)=\{0\}$ (reduced homology) for every $q=0,1,2, \ldots$ Let us prove this for $q \geqslant 1$. Indeed if $T \in$ $\mathbf{I}_{q}\left(Y \cap U^{\prime}\right)$ and $\partial T=0$ then $S=H_{\#}(\llbracket 0,1 \rrbracket \times T) \in \mathbf{I}_{q+1}\left(Y \cap U^{\prime}\right)$ and $([11,4.1 .9])$

$$
\partial S=H_{\#}\left(\boldsymbol{\delta}_{1} \times T\right)-H_{\#}\left(\boldsymbol{\delta}_{0} \times T\right)-H_{\#}(\llbracket 0,1 \rrbracket \times \partial T)=T
$$

because on the one hand $H_{\#}\left(\boldsymbol{\delta}_{0} \times T\right)=0$, $\mathbb{P}$ One has

$$
\begin{aligned}
\mathscr{H}^{q}\left(\operatorname{spt} H_{\#}\left(\boldsymbol{\delta}_{0} \times T\right)\right) & \leqslant \mathscr{H}^{q}(H(\{0\} \times \operatorname{spt} T)) \\
& =\mathscr{H}^{q}(\{y\}) \\
& =0(\text { if } q \geqslant 1)
\end{aligned}
$$


and the conclusion follows since $H_{\#}\left(\boldsymbol{\delta}_{0} \times T\right) \in \mathscr{R}_{q}\left(\mathbb{R}^{n}\right)$. Q $\mathbf{Q}$ and on the other hand $H_{\#}\left(\boldsymbol{\delta}_{1} \times T\right)=T$ because $H(t, \zeta)=\zeta$ whenever $(t, \zeta) \in \operatorname{spt}\left(\boldsymbol{\delta}_{1} \times T\right) \subseteq\{1\} \times U^{\prime}$ and [11, 4.1.15].

We state the following without its obvious proof. It is relevant to notice that the homology theory $\overline{\mathbf{H}}$ satisfies the axioms of Eilenberg-Steenrod, [5].

8.4. Lemma. - Let $Y \subseteq \mathbb{R}^{n}$ be $(\overline{\mathbf{H}}, m)$ locally connected at $y \in Y$ and $f: \mathbb{R}^{n} \rightarrow \mathbb{R}^{n}$ be a Lipschitzian map which is a bi-Lipschitzian homeomorphism of a neighborhood of $y$ in $Y$ onto its image. Then $f(Y)$ is $(\overline{\mathbf{H}}, m)$ locally connected at $f(y)$.

Whenever $W \in \mathbf{G}(n, m)$ we let $P_{W}: \mathbb{R}^{n} \rightarrow \mathbb{R}^{n}$ denote the nearest point projection on $W$. For $W_{1}, W_{2} \in \mathbf{G}(n, m)$ we $\operatorname{define} \operatorname{dist}\left(W_{1}, W_{2}\right)=\left\|P_{W_{1}}-P_{W_{2}}\right\|$ (the operator norm) and we will use the following equality

$$
\operatorname{dist}\left(W_{1}, W_{2}\right)=\max \left\{\operatorname{dist}\left(z, W_{2}\right): z \in W_{1} \cap \mathbf{B}(0,1)\right\} .
$$

Notice that $\operatorname{dist}\left(W_{1}, W_{2}\right) \leqslant 1$ with equality if and only if $W_{1} \cap W_{2}^{\perp} \neq\{0\}$. In Section 14 we will refer to the following.

8.5. Lemma. - Let $V_{0}, V_{1}, V_{2} \in \mathbf{G}(3,2)$ be such that $\operatorname{dim} V_{0} \cap V_{1} \cap V_{2}=0$. Then

$$
\operatorname{dist}\left(V_{0} \cap V_{1}, V_{0} \cap V_{2}\right) \leqslant \operatorname{dist}\left(V_{1}, V_{2}\right)\left(1+\frac{1}{\max \left\{\operatorname{dist}\left(V_{0}, V_{1}\right), \operatorname{dist}\left(V_{0}, V_{2}\right)\right\}}\right) .
$$

Proof. - We choose $e \in V_{0} \cap\left(V_{0} \cap V_{2}\right)^{\perp}$ such that $|e|=1$ and we let $P_{V_{2}, e}: \mathbb{R}^{3} \rightarrow \mathbb{R}^{3}$ denote the "projection on $V_{2}$ parallel to $e$ ". A simple computation reveals that

$$
P_{V_{2}, e}(v)=P_{V_{2}}(v)-\frac{\left\langle v, e^{*}\right\rangle}{\left\langle e, e^{*}\right\rangle} P_{V_{2}}(e),
$$

$v \in \mathbb{R}^{3}$, where $e^{*} \in V_{2}^{\perp}$ has norm 1 . One easily checks that $P_{V_{2}, e}(v) \in V_{0}$ whenever $v \in V_{0}$. Now if $z \in V_{0} \cap V_{1} \cap \mathbf{B}(0,1)$ then $P_{V_{2}, z} \in V_{0} \cap V_{2}$ and therefore

$$
\begin{aligned}
\operatorname{dist}\left(z, V_{0} \cap V_{2}\right) & \leqslant\left|z-P_{V_{2}, e}(z)\right| \\
& \leqslant\left|z-P_{V_{2}}(z)\right|+\left|P_{V_{2}}(z)-P_{V_{2}, e}(z)\right| \\
& =\operatorname{dist}\left(z, V_{2}\right)+\frac{\left|\left\langle z, e^{*}\right\rangle\right|}{\left|\left\langle e, e^{*}\right\rangle\right|}\left|P_{V_{2}}(e)\right| .
\end{aligned}
$$

Readily $\left|\left\langle z, e^{*}\right\rangle\right|=\left|P_{V_{2}^{\perp}}(z)\right|=\operatorname{dist}\left(z, V_{2}\right)$ and $\left|\left\langle e, e^{*}\right\rangle\right|=\operatorname{dist}\left(V_{0}, V_{2}\right)$. Consequently

$$
\begin{aligned}
\operatorname{dist}\left(z, V_{0} \cap V_{2}\right) & \leqslant \operatorname{dist}\left(z, V_{2}\right)\left(1+\frac{1}{\operatorname{dist}\left(V_{0}, V_{2}\right)}\right) \\
& \leqslant \operatorname{dist}\left(V_{1}, V_{2}\right)\left(1+\frac{1}{\operatorname{dist}\left(V_{0}, V_{2}\right)}\right),
\end{aligned}
$$

and the conclusion follows from the arbitrariness of $z \in V_{0} \cap V_{1} \cap \mathbf{B}(0,1)$ and the symmetric role of $V_{1}$ and $V_{2}$.

The following is well-known. It says that if one sees a Lipschitz graph and tilts one's head a small angle, then one still sees a Lipschitz graph.

8.6. Lemma. - Assume that

$4^{\mathrm{e}}$ SÉRIE - TOME $42-2009-\mathrm{N}^{\mathrm{o}} 1$ 
(A) $W_{1}, W_{2} \in \mathbf{G}(n, m)$;

(B) $r>0, f: W_{1} \cap \mathbf{U}(0, r) \rightarrow W_{1}^{\perp}$ is Lipschitzian, $f(0)=0$ and $S=\operatorname{graph}(f)$;

(C) $\operatorname{Lip} f<1$ and $\operatorname{dist}\left(W_{1}, W_{2}\right)<1-\operatorname{Lip} f$.

Then 0 belongs to the interior of $P_{W_{2}}(S)$ relative to $W_{2}$ and there exists a Lipschitzian map $g: W_{2} \cap P_{W_{2}}(S) \rightarrow W_{2}^{\perp}$ such that $\operatorname{graph}(g)=S$.

Proof. - The proof of the existence of a Lipschitzian map $g$ can be based on [4, Lemma 8.2]. Indeed it suffices to show that for every $x \in S$ and every $\rho>0$ one has $\mathbf{B}(x, \rho) \subseteq \mathbf{B}(x+$ $\left.W_{2}, \sigma \rho\right)$ for some appropriate $0<\sigma<1$. Let $x^{\prime} \in S \cap \mathbf{B}(x, \rho)$ and abbreviate $\xi=P_{W_{1}}(x)$ and $\xi^{\prime}=P_{W_{1}}\left(x^{\prime}\right)$. Readily

$$
\begin{aligned}
\operatorname{dist}\left(x^{\prime}, x+W_{2}\right) & =\operatorname{dist}\left(x^{\prime}-x, W_{2}\right) \\
& \leqslant\left|\left(x^{\prime}-x\right)-\left(\xi^{\prime}-\xi\right)\right|+\operatorname{dist}\left(\xi^{\prime}-\xi, W_{2}\right) \\
& \leqslant\left|f\left(\xi^{\prime}\right)-f(\xi)\right|+\rho \operatorname{dist}\left(W_{1}, W_{2}\right)
\end{aligned}
$$

and the conclusion holds with $\sigma:=\operatorname{Lip} f+\operatorname{dist}\left(W_{1}, W_{2}\right)$. Finally, letting $\tilde{f}(w)=w+f(w)$, $w \in W_{1} \cap \mathbf{U}(0, r)$, and $\tilde{g}(w)=w+g(w), w \in P_{W_{2}}(S)$, one checks that $P_{W_{2}} \circ \tilde{f}$ and $P_{W_{1}} \circ \tilde{g}$ are inverse of each other and both Lipschitzian. This implies that 0 is an interior point of $P_{W_{2}}(S)$.

\section{PART II \\ APPROXIMATION: THE CASE OF NO BOUNDARY}

\section{Central projections}

We start with two notations. If $a, b \in \mathbb{R}^{n}$ then we set $[a, b]=\mathbb{R}^{n} \cap\{a+t(b-a): 0 \leqslant t \leqslant 1\}$. Next, with each $a \in \mathbb{R}^{n}$ and $u \in \mathbb{R}^{n} \backslash\{0\}$ we associate $L_{a, u}=\mathbb{R}^{n} \cap\{a+t u: t \geqslant 0\}$.

Let $C \subseteq \mathbb{R}^{n}$ be a compact convex set with $\operatorname{Int} C \neq \varnothing$. Given $a \in \operatorname{Int} C$ and $u \in \mathbb{R}^{n} \backslash\{0\}$ we note that $L_{a, u} \cap \operatorname{Bdry} C$ is a singleton. P First notice that $L_{a, u} \cap C$ is a compact convex subset of $L_{a, u}$ containing $a$, therefore $L_{a, u} \cap C=[a, b]$ for some $b \in C$. Since $a \in \operatorname{Int} C$ it follows easily that $[a, b] \backslash\{b\} \subseteq \operatorname{Int} C$ and, in turn, that $L_{a, u} \cap \operatorname{Bdry} C=\{b\}$. Q

9.1. Definition. - Let $C \subseteq \mathbb{R}^{n}$ be a compact convex set with $\operatorname{Int} C \neq \varnothing$, and $a \in \operatorname{Int} C$. For each $x \in C \backslash\{a\}$ we define $\pi_{C, a}(x) \in \mathbb{R}^{n}$ by the requirement that

$$
L_{a, x-a} \cap \operatorname{Bdry} C=\left\{\pi_{C, a}(x)\right\} .
$$

We will now study the (local) modulus of continuity of the map

$$
\pi_{C, a}: C \backslash\{a\} \rightarrow \text { Bdry } C .
$$

The following is rather trivial and better understood with the help of a drawing.

9.2. Lemma. - Assume that:

(A) $V$ is a 2-dimensional Hilbert space and $e_{1}, e_{2}$ is an orthonormal basis of $V$;

(B) $0<\lambda \leqslant 1<\Lambda<\infty$ and $x=\lambda e_{1}$; 
(C) $h \in V,\left\langle h, e_{2}\right\rangle \geqslant 0$ and

$$
|h| \leqslant \frac{1}{2} \frac{\lambda}{\Lambda} \sqrt{\frac{\Lambda-1}{\Lambda+1}} .
$$

Then the following conclusions hold:

(D) There exist $\xi_{1}, \xi_{2} \in V$ such that $\left|\xi_{1}\right|=\left|\xi_{2}\right|=1,\left\langle\xi_{i}, \Lambda e_{1}-\xi_{i}\right\rangle=0, i=1,2$, and $\left\langle\xi_{1}, e_{2}\right\rangle>0,\left\langle\xi_{2}, e_{2}\right\rangle<0$

(E) There exist $\zeta_{1}, \zeta_{2} \in V$ such that

$$
L_{0, x+h} \cap\left[\xi_{1}, \Lambda e_{1}\right]=\left\{\zeta_{1}\right\}
$$

and

$$
L_{0, x+h} \cap L_{\xi_{2}, \Lambda e_{1}-\xi_{2}}=\left\{\zeta_{2}\right\}
$$

(F) $-\Lambda \sqrt{\Lambda^{2}-1} \frac{|h|}{|x|-|h|} \leqslant\left\langle\zeta_{1}, e_{1}\right\rangle-\Lambda \leqslant 0$;

(G) $0 \leqslant\left\langle\zeta_{2}, e_{1}\right\rangle-\Lambda \leqslant \Lambda \sqrt{\Lambda^{2}-1} \frac{|h|}{|x|-\Lambda|h|}$;

(H) For each $\zeta \in\left[\zeta_{1}, \zeta_{2}\right]$ one has

$$
\left|\left\langle\zeta-\Lambda e_{1}, e_{1}\right\rangle\right| \leqslant \Lambda \sqrt{\Lambda^{2}-1} \frac{|h|}{|x|-\Lambda|h|} ;
$$

(I) If we assume furthermore that

(i) $V \subseteq \mathbb{R}^{n}$;

(ii) $C \subseteq \mathbb{R}^{n}$ is compact, convex, and $0 \in \operatorname{Int} C$;

(iii) $\xi_{1}, \xi_{2}, x, x+h \in C$;

(iv) $\pi_{C, 0}(x)=\Lambda e_{1}$

$$
\text { then }
$$

$$
\pi_{C, 0}(x+h) \in\left[\zeta_{1}, \zeta_{2}\right] .
$$

Proof. - It is trivial to check that there are $\xi_{1}, \xi_{2} \in V$ verifying the conditions stated in (D) and that

$$
\left\langle\xi_{1}, e_{1}\right\rangle=\left\langle\xi_{2}, e_{1}\right\rangle=\frac{1}{\Lambda}
$$

and

$$
\begin{aligned}
& \left\langle\xi_{1}, e_{2}\right\rangle=\frac{\sqrt{\Lambda^{2}-1}}{\Lambda} \\
& \left\langle\xi_{2}, e_{2}\right\rangle=-\frac{\sqrt{\Lambda^{2}-1}}{\Lambda} .
\end{aligned}
$$

In order to prove (E) we seek pairs $\left(t_{i}, s_{i}\right), i=1,2$, such that

$$
\xi_{i}+t_{i}\left(\Lambda e_{1}-\xi_{i}\right)=s_{i}\left(\lambda e_{1}+h\right), i=1,2 .
$$

We easily check that the upper bound on $|h|$ stated in hypothesis (C) guarantees the existence of solutions for these two systems of linear equations. It remains to show that
(a) $s_{1} \geqslant 0$
(b) $s_{2} \geqslant 0$;
(c) $t_{1} \leqslant 1$;
(d) $0 \leqslant t_{1}$
(e) $t_{2} \geqslant 1$. 
In order to establish (a) and (b) we take the inner product of (4) with $\xi_{i}, i=1,2$, and we plug (2) in the resulting equation to infer that $1=s_{i}\left(\lambda \Lambda^{-1}+\left\langle h, \xi_{i}\right\rangle\right)$, which yields our conclusions on noticing that $|h| \leqslant \frac{1}{2} \lambda \Lambda^{-1}$ according to hypothesis (C). Next we take the inner product of (4) with $e_{2}$, which yields $\left\langle\xi_{i}, e_{2}\right\rangle\left(1-t_{i}\right)=s_{i}\left\langle h, e_{2}\right\rangle, i=1,2$, and we see that (c) and (e) now follow from (a) and (b) respectively. Finally we prove (d) as follows. We take the inner product of (4) with $\Lambda e_{1}-\xi_{1}$ to infer that

$$
t_{1}\left|\Lambda e_{1}-\xi_{1}\right|^{2}=s_{1}\left(\lambda\left(\Lambda-\Lambda^{-1}\right)+\Lambda\left\langle h, e_{1}\right\rangle-\left\langle h, \xi_{1}\right\rangle\right)
$$

and we use hypothesis $(\mathrm{C})$ again to show that the factor of $s_{1}$ is nonnegative.

We now turn to proving (F). We notice from (C) that $|h| \leqslant \frac{1}{2}|x|$ and we infer from (4) and (c) and (d) above that

$$
(|x|-|h|) s_{1} \leqslant s_{1}|x+h|=\left|\xi_{1}\left(1-t_{1}\right)+t_{1} \Lambda e_{1}\right| \leqslant\left(1-t_{1}\right)+t_{1} \Lambda \leqslant \Lambda
$$

therefore

$$
s_{1} \leqslant \frac{\Lambda}{|x|-|h|} .
$$

Next we take the inner product of (4) with $e_{2}$ :

$$
\left\langle\xi_{1}, e_{2}\right\rangle\left(1-t_{1}\right)=s_{1}\left\langle h, e_{2}\right\rangle
$$

and we use (3) together with (5) to conclude that

$$
0 \leqslant 1-t_{1} \leqslant \frac{\Lambda^{2}}{\sqrt{\Lambda^{2}-1}} \frac{|h|}{|x|-|h|} .
$$

Finally, taking the inner product of (4) with $e_{1}$ yields

$$
\left\langle\xi_{1}, e_{1}\right\rangle\left(1-t_{1}\right)+t_{1} \Lambda=\left\langle\zeta_{1}, e_{1}\right\rangle
$$

in other words

$$
\left\langle\zeta_{1}, e_{1}\right\rangle-\Lambda=\left(\frac{1}{\Lambda}-\Lambda\right)\left(1-t_{1}\right)
$$

and conclusion (F) now immediately follows from (6).

In order to prove (G) we first take the inner product of (4) with $\xi_{2}$ and referring to (2) we obtain

$$
1=s_{2}\left(\lambda \frac{1}{\Lambda}+\left\langle h, \xi_{2}\right\rangle\right) \geqslant s_{2}\left(\frac{1}{\Lambda}|x|-|h|\right),
$$

therefore

$$
s_{2} \leqslant \frac{\Lambda}{|x|-\Lambda|h|}
$$

because $\Lambda|h| \leqslant \frac{1}{2}|x|$ according to (C). Taking the inner product of (4) with $e_{2}$ we see that

$$
\left\langle\xi_{2}, e_{2}\right\rangle\left(1-t_{2}\right)=s_{2}\left\langle h, e_{2}\right\rangle
$$

which together with (7), (3) and (e) yields

$$
0 \leqslant t_{2}-1 \leqslant \frac{\Lambda^{2}}{\sqrt{\Lambda^{2}-1}} \frac{|h|}{|x|-\Lambda|h|} .
$$

Finally taking the inner product of (4) with $e_{1}$ yields

$$
\left\langle\xi_{2}, e_{1}\right\rangle\left(1-t_{2}\right)+t_{2} \Lambda=\left\langle\zeta_{2}, e_{1}\right\rangle
$$


in other words,

$$
\left\langle\zeta_{2}, e_{1}\right\rangle-\Lambda=\left(\frac{1}{\Lambda}-\Lambda\right)\left(1-t_{2}\right)
$$

and conclusion $(\mathrm{G})$ is now a consequence of (8).

We notice that $(\mathrm{H})$ trivially follows from $(\mathrm{F})$ and $(\mathrm{G})$. In order to prove (I) we let $\zeta=$ $\pi_{C, 0}(x+h)$. Suppose $|\zeta|>\left|\zeta_{2}\right|$. Since $\left[\zeta, \xi_{2}\right] \subseteq C$ we would have that if $\left\{\zeta^{\prime}\right\}=\left[\zeta, \xi_{2}\right] \cap L_{0, e_{1}}$ then $\zeta^{\prime} \in C$, but $\left|\zeta^{\prime}\right|>\Lambda=\left|\pi_{C, 0}(x)\right|$, a contradiction. Therefore $|\zeta| \leqslant\left|\zeta_{2}\right|$. Furthermore, since $\xi_{1} \in C$ we have $\left[\xi_{1}, \pi_{C, 0}(x)\right] \subseteq C$, whence also $\zeta_{1} \in C$, and in turn $\left|\zeta_{1}\right| \leqslant|\zeta|$.

The above lemma was designed to estimate the local Lipschitz constant of $\pi_{C, a}$. We now state the relevant definition and result.

9.3. Definition. - Let $\left(X, d_{X}\right)$ and $\left(Z, d_{Z}\right)$ be metric spaces, $f: X \rightarrow Z$ and $x \in X$. We define the local Lipschitz constant of $f$ at $x$ as follows

$$
\operatorname{Lip}_{x} f=\limsup _{r \downarrow 0}\left\{\frac{d_{Z}(f(x), f(y))}{d_{X}(x, y)}: y \in \mathbf{B}(x, r) \backslash\{x\}\right\} .
$$

9.4. Remark. - Given an open ball $U \subseteq \mathbb{R}^{n}$ and $f: \mathbb{R}^{n} \rightarrow \mathbb{R}$ we infer that

$$
\operatorname{Lip} f\left\lceil U=\sup \left\{\operatorname{Lip}_{x} f: x \in U\right\}\right. \text {. }
$$

\subsection{Corollary. - Assume that:}

(A) $C \subseteq \mathbb{R}^{n}$ is compact convex with $\operatorname{Int} C \neq \varnothing$;

(B) $a \in \operatorname{Int} C$;

(C) $0<r<R$ and $\mathbf{B}(a, r) \subseteq \operatorname{Int} C \subseteq \mathbf{B}(a, R)$;

(D) $x \in C \backslash\{a\}$.

Then

$$
\operatorname{Lip}_{x} \pi_{C, a} \leqslant \frac{R^{2}}{r} \frac{1}{|x-a|}
$$

Proof. - It is clearly sufficient to prove it in case $a=0$. We assume first that $r=1$. We let $W \in \mathbf{G}(n, n-1)$ be such that $W=\mathbb{R}^{n} \cap\{z:\langle z, x\rangle=0\}$. We observe that for each $y \in C \backslash\{0\}:$

$$
\begin{aligned}
\left|P_{W}\left(\pi_{C, 0}(y)\right)-P_{W}\left(\pi_{C, 0}(x)\right)\right| & =\left|P_{W}\left(\pi_{C, 0}(y)\right)\right|=\left|P_{W}\left(y|y|^{-1}\left|\pi_{C, 0}(y)\right|\right)\right| \\
& \leqslant R|y|^{-1}\left|P_{W}(y)\right|=R|y|^{-1}\left|P_{W}(y-x)\right| \\
& \leqslant R|y|^{-1}|y-x| .
\end{aligned}
$$

On the other hand, assuming that $y$ and $x$ are linearly independent we may apply Lemma 9.2 with $V=\operatorname{span}\{y, x\}, \lambda=|x|, \Lambda=\left|\pi_{C, 0}(x)\right|$ (notice that $1<\Lambda \leqslant R$ ), $e_{1}=x|x|^{-1}$, $h=y-x$. If $|y-x| \leqslant \frac{|x|}{2 \Lambda} \sqrt{\frac{\Lambda-1}{\Lambda+1}}=: \gamma$ then it follows from Lemma 9.2(H) and (I) (recall that $\left.\xi_{1}, \xi_{2} \in \mathbf{B}(0,1) \subseteq C\right)$ that

$$
\begin{aligned}
\left|\left\langle\pi_{C, 0}(y)-\pi_{C, 0}(x), x|x|^{-1}\right\rangle\right| & \leqslant \Lambda \sqrt{\Lambda^{2}-1} \frac{|y-x|}{|x|-\Lambda|y-x|} \\
& \leqslant R \sqrt{R^{2}-1} \frac{|y-x|}{|x|-R|y-x|} .
\end{aligned}
$$


From (9) and (10) we now infer that

$$
\left|\pi_{C, 0}(y)-\pi_{C, 0}(x)\right|^{2} \leqslant|y-x|^{2}\left(\frac{R^{2}}{|y|^{2}}+\frac{R^{2}\left(R^{2}-1\right)}{(|x|-R|y-x|)^{2}}\right)
$$

provided $y \in C \cap \mathbf{B}(x, \gamma) \backslash W^{\perp}$. Letting $y \rightarrow x$ in (11) we obtain

$$
\operatorname{Lip}_{x} \pi_{C, 0} \leqslant \frac{R^{2}}{|x|}
$$

This proves the corollary in case $r=1$. For $r \neq 1$ we notice that $\pi_{C, 0}(z)=r \pi_{r^{-1} C, 0}\left(r^{-1} z\right)$ whenever $z \in C \backslash\{0\}$ and we apply (12) with $r^{-1} C, r^{-1} R$ and $r^{-1} x$ in place of $C, R$ and $x$ :

$$
\begin{aligned}
\left|\pi_{C, 0}(y)-\pi_{C, 0}(x)\right| & =r\left|\pi_{r^{-1} C, 0}\left(r^{-1} y\right)-\pi_{r^{-1} C, 0}\left(r^{-1} x\right)\right| \\
& \leqslant r\left(\left|r^{-1} y-r^{-1} y\right|+o\left(\left|r^{-1} y-r^{-1} x\right|\right)\right) \frac{\left(r^{-1} R\right)^{2}}{\left|r^{-1} x\right|} \\
& =\left(|y-x|+o\left(\left|r^{-1} y-r^{-1} x\right|\right)\right) \frac{R^{2}}{r} \frac{1}{|x|} .
\end{aligned}
$$

The conclusion follows on letting $y \rightarrow x$.

9.6. Remark. - When $C=\mathbf{B}(0, R)$, letting $r \rightarrow R$ we find that $\operatorname{Lip}_{x} \pi_{C, 0} \leqslant \frac{R}{|x|}$. Since $\pi_{C, 0}(z)=R \frac{z}{|z|}$, one easily checks that in fact $\operatorname{Lip}_{x} \pi_{C, 0}=\frac{R}{|x|}$. Furthermore we notice that the corollary and its proof remain valid in infinite dimensional Hilbert spaces.

\subsection{Proposition. - Assume that}

(A) $C \subseteq \mathbb{R}^{n}$ is compact convex and $a \in \operatorname{Int} C$;

(B) $0<r<R$ and $\mathbf{B}(a, r) \subseteq \operatorname{Int} C \subseteq \mathbf{B}(a, R)$;

(C) $0<m \leqslant n-1$ and $E \subseteq C$ is $\mathscr{H}^{m}$ measurable with $\mathscr{H}^{m}(E)<\infty$.

Then there exists $b \in \mathbf{B}(a, r) \backslash E$ such that

$$
\mathscr{H}^{m}\left(\pi_{C, b}(E)\right) \leqslant \frac{n 2^{n-m+1}}{n-m}\left(\frac{R}{r}\right)^{n+m} \mathscr{H}^{m}(E) .
$$

Proof. - For use in the present proof we define the function

$$
\operatorname{Lip}: \mathbf{B}(a, r) \times C \rightarrow \mathbb{R}:(b, x) \mapsto \frac{R^{2}}{r} \frac{1}{|x-b|}
$$

which is readily Borel measurable, and continuous on

$$
Z:=\mathbf{B}(a, r) \times C \cap\{(b, x): b \neq x\}) .
$$


Let $\mu=\mathscr{H}^{m}\left\llcorner E\right.$. Applications of Tonelli's Theorem below will show that Lip is $\mathscr{L}^{n} \times \mu$ summable. Indeed, letting $\tau=R / r$ we note that

$$
\begin{aligned}
\int_{\mathbf{B}(a, r)} d \mathscr{L}^{n}(b) \int_{C} \operatorname{Lip}(b, x)^{m} d \mu(x) & =\int_{\mathbf{B}(a, r)} d \mathscr{L}^{n}(b) \int_{C}\left(\frac{R^{2}}{r} \frac{1}{|x-b|}\right)^{m} d \mu(x) \\
& =\tau^{2 m} r^{m} \int_{C} d \mu(x) \int_{\mathbf{B}(a, r)} \frac{1}{|x-b|^{m}} d \mathscr{L}^{n}(b) \\
& \leqslant \tau^{2 m} r^{m} \int_{C} d \mu(x) \int_{\mathbf{B}(x, 2 R)} \frac{1}{|x-b|^{m}} d \mathscr{L}^{n}(b) \\
& =\tau^{2 m} r^{m} \mu(C) \frac{n \boldsymbol{\alpha}(n)(2 R)^{n-m}}{n-m} \\
& =\tau^{2 m} r^{m} \mu(C) \frac{n \boldsymbol{\alpha}(n) 2^{n-m} \tau^{n-m}}{n-m} r^{n-m} \\
& =\frac{\tau^{n+m} n 2^{n-m}}{n-m} \mu(C) \mathscr{L}^{n}(\mathbf{B}(a, r)) .
\end{aligned}
$$

Since $\mathscr{L}^{n}(E)=0$, it readily follows from (13) that there exists $b \in \mathbf{B}(a, r) \backslash E$ such that

$$
\int_{C} \operatorname{Lip}(b, x)^{m} d \mu(x) \leqslant \frac{\tau^{n+m} n 2^{n-m+1}}{n-m} \mu(C) .
$$

The proposition now becomes a consequence of the inequality

$$
\mathscr{H}^{m}\left(\pi_{C, b}(E)\right) \leqslant \int_{C} \mathbf{L i p}(b, x)^{m} d\left(\mathscr{H}^{m}\llcorner E)(x)\right.
$$

which we will establish subsequently.

We start by observing that if $N \subseteq E$ and $\mathscr{H}^{m}(N)=0$ then $\mathscr{H}^{m}\left(\pi_{C, b}(N)\right)=0$. 田 Indeed for each $x \in N$ there exists $r(x)>0$ such that $\operatorname{Lip}\left(\pi_{C, b}\lceil\mathbf{B}(x, r(x))) \leqslant 1+\operatorname{Lip}_{x} \pi_{C, b}\right.$. Since $N$ is Lindelöf there is a sequence $x_{1}, x_{2}, \cdots \in N$ such that $N \subseteq \cup_{i=1}^{\infty} N \cap \mathbf{B}\left(x_{i}, r\left(x_{i}\right)\right)$. Therefore

$$
\mathscr{H}^{m}\left(\pi_{C, b}(N)\right) \leqslant \sum_{i=1}^{\infty} \operatorname{Lip}\left(\pi_{C, b}\left\lceil\mathbf{B}\left(x_{i}, r\left(x_{i}\right)\right)\right)^{m} \mathscr{H}^{m}\left(N \cap \mathbf{B}\left(x_{i}, r\left(x_{i}\right)\right)\right)=0 .\right.
$$

Next we fix $j=1,2, \ldots$ and we infer from the Besicovitch-Vitali covering Theorem, [11, 2.8.15], that there exists a (at most countable) disjoint collection of balls $\mathbf{B}\left(x_{j, i}, r_{j, i}\right), i \in I_{j}$, such that $\left\{x_{j, i}: i \in I_{j}\right\} \subseteq E, \sup \left\{r_{j, i}: i \in I_{j}\right\} \leqslant j^{-1}$ and $\mathscr{H}^{m}\left(N_{j}\right)=0$ where

$$
N_{j}=E \backslash \cup_{i \in I_{j}} \mathbf{B}\left(x_{j, i}, r_{j, i}\right) \text {. }
$$

Therefore

$$
\begin{aligned}
\mathscr{H}^{m}\left(\pi_{C, b}(E)\right) & \leqslant \sum_{i \in I_{j}} \mathscr{H}^{m}\left(\pi_{C, b}\left(E \cap \mathbf{B}\left(x_{j, i}, r_{j, i}\right)\right)\right) \\
& \leqslant \int_{\mathbb{R}^{n}} \sum_{i \in I_{j}}\left(\operatorname { L i p } \pi _ { C , b } \lceil \mathbf { B } ( x _ { j , i } , r _ { j , i } ) ) ^ { m } \mathbb { 1 } _ { \mathbf { B } ( x _ { j , i } , r _ { j , i } ) } d \left(\mathscr{H}^{m}\llcorner E) .\right.\right.
\end{aligned}
$$

Denote by $f_{j}$ the integrand in the last line of the above inequality. Put $N=\cup_{j=1}^{\infty} N_{j}$ so that $\mathscr{H}^{m}(N)=0$ and notice that for each $x \in E \backslash N$ and each $j=1,2, \ldots$ there is a unique 
$i(j, x) \in I_{j}$ such that $x \in \mathbf{B}\left(x_{j, i(j, x)}, r_{j, i(j, x)}\right)$, whence

$$
\begin{aligned}
\liminf _{j \rightarrow \infty} f_{j}(x) & =\liminf _{j \rightarrow \infty}\left(\operatorname{Lip} \pi_{C, b}\left\lceil\mathbf{B}\left(x_{j, i(j, x)}, r_{j, i(j, x)}\right)\right)^{m}\right. \\
& \leqslant \liminf _{j \rightarrow \infty}\left(\operatorname{Lip} \pi_{C, b}\left\lceil\mathbf{B}\left(x, 2 j^{-1}\right)\right)^{m}\right. \\
& =\liminf _{j \rightarrow \infty}\left(\sup \left\{\operatorname{Lip}_{\xi} \pi_{C, b}: \xi \in \mathbf{B}\left(x, 2 j^{-1}\right)\right\}\right)^{m} \\
& \leqslant \liminf _{j \rightarrow \infty}\left(\sup \left\{\operatorname{Lip}(b, \xi): \xi \in \mathbf{B}\left(x, 2 j^{-1}\right)\right\}\right)^{m} \\
& =\mathbf{L i p}(b, x)^{m}
\end{aligned}
$$

according to Corollary 9.5 and the continuity of Lip at $(b, x) \in Z$. Plugging this into (16) and referring to Fatou's Lemma we obtain

$$
\begin{aligned}
\mathscr{H}^{m}\left(\pi_{C, b}(E)\right) & \leqslant \liminf _{j \rightarrow \infty} \int_{\mathbb{R}^{n}} f_{j}(x) d\left(\mathscr{H}^{m}\llcorner E)(x)\right. \\
& \leqslant \int_{\mathbb{R}^{n}} \liminf _{j \rightarrow \infty} f_{j}(x) d\left(\mathscr{H}^{m}\llcorner E)(x)\right. \\
& \leqslant \int_{\mathbb{R}^{n}} \operatorname{Lip}(b, x)^{m} d\left(\mathscr{H}^{m}\llcorner E)(x) .\right.
\end{aligned}
$$

Whence (15) is established and the proof is complete.

\section{Homotopy equivalence in the Lipschitzian category}

Here we state and prove some easy results to be used in the next section. Given $x, y \in \mathbb{R}^{n}$ we call path joining $x$ and $y$ a continuous function $\gamma:[0,1] \rightarrow \mathbb{R}^{n}$ such that $\gamma(0)=x$ and $\gamma(1)=x$. We say that $\gamma$ is a path in $Z$, for some $Z \subseteq \mathbb{R}^{n}$, if $\operatorname{im} \gamma \subseteq Z$. Finally we recall that

$$
\operatorname{length}(\gamma)=\sup \left\{\sum_{k=1}^{\kappa}\left|\gamma\left(t_{k}\right)-\gamma\left(t_{k-1}\right)\right|: 0=t_{0}<\cdots<t_{\kappa}=1\right\} \text {. }
$$

Whenever $\gamma$ is injective one has length $(\gamma)=\mathscr{H}^{1}(\operatorname{im} \gamma)$.

10.1. Definition. - Let $S \subseteq \mathbb{R}^{n}$ be such that $\mathbb{R}^{n} \backslash S$ is arcwise connected. For each $x, y \in \mathbb{R}^{n} \backslash S$ we let

$$
d_{S}(x, y)=\inf \left\{\operatorname{length}(\gamma): \gamma \text { is a path in } \mathbb{R}^{n} \backslash S \text { joining } x \text { and } y\right\} .
$$

It is most obvious that $|x-y| \leqslant d_{S}(x, y), x, y \in \mathbb{R}^{n} \backslash S$. In some cases $d_{S}$ is a metric equivalent to the restriction of the Euclidean metric, for instance under the following hypothesis.

10.2. Lemma. - Let $S_{1}, \ldots, S_{\kappa}$ be disjoint compact convex sets with nonempty interiors, and $S=\cup_{k=1}^{\kappa} S_{k}$. There exists $C>0$ such that

$$
d_{S}(x, y) \leqslant C|x-y| .
$$


Proof. - We start by observing that $d_{\operatorname{Int} S}=d_{S}$. Next we select $a_{k} \in \operatorname{Int} S_{k}, 0<r_{k} \leqslant R_{k}$ such that $\mathbf{B}\left(a_{k}, r_{k}\right) \subseteq \operatorname{Int} S_{k} \subseteq \mathbf{B}\left(a_{k}, R_{k}\right), k=1, \ldots, \kappa$. Given $x, y \in \mathbb{R}^{n} \backslash S$ we let $\gamma$ denote an arclength parametrization of the line segment joining $x$ and $y$, so that length $(\gamma)=|x-y|$. Denoting by $K$ the collection of those indexes $k=1, \ldots, \kappa$ such that Int $S_{k} \cap[x, y] \neq \varnothing$ and, for each $k \in K$, by $\left[s_{k}, t_{k}\right]$ the interval $\gamma^{-1}\left(S_{k} \cap[x, y]\right)$, we define a new path $\gamma^{\prime}$ in $\mathbb{R}^{n} \backslash \operatorname{Int} S$ joining $x$ and $y$ in the following way: we replace each $\gamma \uparrow\left[s_{k}, t_{k}\right], k \in K$, with $\pi_{S_{k}, b_{k}} \circ \gamma$ where $b_{k}$ is as in the conclusion of Proposition 9.7 (applied with $m=1$ and $E=S_{k} \cap[x, y]$ ). Since $d_{\operatorname{Int} S}(x, y) \leqslant \operatorname{length}\left(\gamma^{\prime}\right)$ we obtain at once

$$
d_{\text {Int } S}(x, y) \leqslant \frac{n 2^{n}}{n-1} \max \left\{\left(\frac{R_{k}}{r_{k}}\right)^{n+1}: k=1, \ldots, \kappa\right\}|x-y| .
$$

10.3. Lemma. - Assume that $\kappa$ is a positive integer and

(A) $C_{k} \subseteq \mathbb{R}^{n}, k=1, \ldots, \kappa$ are compact convex sets whose interiors are pairwise disjoint;

(B) $b_{k} \in \mathbb{R}^{n}, \sigma_{k}>0$ and $\mathbf{B}\left(b_{k}, \sigma_{k}\right) \subseteq \operatorname{Int} C_{k}, k=1, \ldots, \kappa$;

(C) $S=\cup_{k=1}^{\kappa} \mathbf{B}\left(b_{k}, \sigma_{k}\right)$ and

$$
H:[0,1] \times \mathbb{R}^{n} \backslash S \rightarrow \mathbb{R}^{n} \backslash S
$$

is defined as follows: $H(t, x)=x+t\left(\pi_{C_{k}, b_{k}}(x)-x\right)$ in case $x \in C_{k} \backslash \mathbf{B}\left(b_{k}, \sigma_{k}\right)$ for some $k=1, \ldots, \kappa$, and $H(t, x)=x$ otherwise.

\section{Then $H$ is Lipschitzian.}

Proof. - Define $f(x)=H(1, x), x \in \mathbb{R}^{n} \backslash S$. Recalling that $\pi_{C_{k}, b_{k}} \uparrow C_{k} \backslash \mathbf{B}\left(b_{k}, \sigma_{k}\right)$ is Lipschitzian (according to Corollary 9.5 together with Lemma 10.2) and that $\pi_{C_{k}, b_{k}}$ । Bdry $C_{k}=\operatorname{id}_{\text {Bdry } C_{k}}$, we infer e.g. from [7, 2.3.2] that for each closed ball $B \subseteq \mathbb{R}^{n} \backslash S$ one has $\operatorname{Lip} f\lceil B \leqslant \Lambda$ where

$$
\Lambda=\max \left\{\operatorname{Lip} \pi_{C_{k}, b_{k}}\left\lceil C_{k} \backslash \mathbf{B}\left(b_{k}, \sigma_{k}\right): k=1, \ldots, \kappa\right\} .\right.
$$

According to Lemma 10.2 and the fundamental theorem of calculus for Lipschitzian function we infer that $\operatorname{Lip} f \leqslant C \Lambda$ where $C=C(S)$ is associated with $S$ in that lemma. Finally one readily checks that

$$
\text { Lip } H \leqslant 2 \max \left\{1+\operatorname{Lip} f, \max \left\{\operatorname{diam} C_{1}, \ldots, \operatorname{diam} C_{k}\right\}\right\} .
$$

We end this section with a slight strengthening of the notion of homotopy.

10.4. Definition. - Let $U \subseteq \mathbb{R}^{n}$ be open and $0<\varepsilon \leqslant \infty$. We say that two Lipschitzian maps $h_{0}, h_{1}: U \rightarrow U$ are $\varepsilon$-homotopic in the Lipschitzian category if there exists a Lipschitzian map

$$
H:[0,1] \times U \rightarrow U
$$

such that $H(0, x)=h_{0}(x), H(1, x)=h_{1}(x)$ and $|H(t, x)-x|<\varepsilon$ for every $x \in U$ and $0 \leqslant t \leqslant 1$. When $\varepsilon=\infty$ we simply say that $h_{0}$ and $h_{1}$ are homotopic in the Lipschitzian category.

10.5. REMARK. - If $h_{0}$ and $h_{1}$ are $\varepsilon$-homotopic then readily $\left|h_{0}(x)-h_{1}(x)\right|<\varepsilon$ for every $x \in U$. In particular $\operatorname{dist}_{\mathscr{H}}\left(h_{0}(A), h_{1}(A)\right) \leqslant \varepsilon$ for every $A \subseteq U$. 
10.6. REMARK. - We notice that if $H$ is an affine homotopy, i.e. $H(t, x)=h_{0}(x)+$ $t\left(h_{1}(x)-h_{0}(x)\right)$, and if $\left|h_{0}(x)-h_{1}(x)\right|<\varepsilon$ for every $x \in U$, then readily $h_{0}$ and $h_{1}$ are $\varepsilon$-homotopic. This observation applies to the homotopy defined in Lemma 10.3.

10.7. Remark. - We leave it to the reader to check that if $h_{0}, h_{1}: U \rightarrow U$ are $\varepsilon$-homotopic in the Lipschitzian category and if $h_{0}^{\prime}, h_{1}^{\prime}: U \rightarrow U$ are $\varepsilon^{\prime}$-homotopic in the Lipschitzian category, then $h_{0}^{\prime} \circ h_{0}$ and $h_{1}^{\prime} \circ h_{1}$ are $\varepsilon^{\prime}+\varepsilon$-homotopic in the Lipschitzian category.

\section{Deformation and approximation theorem}

The approximating sets will be of the type described in the next definition.

11.1. Definition. - Let $m \in\{1, \ldots, n-1\}$. A set $P \subseteq \mathbb{R}^{n}$ is $m$-dimensional polyhedral provided there exist finitely many $m$-dimensional linear subspaces $W_{k} \in \mathbf{G}(n, m), x_{k} \in \mathbb{R}^{n}$, and relatively open ${ }^{(14)}$ sets $Z_{k} \subseteq x_{k}+W_{k}, k=1, \ldots, \kappa$, such that

$$
P=\cup_{k=1}^{\kappa} Z_{k} \text {. }
$$

In order that the argument to be made in Part 3 runs smoothly we will also require that the polyhedral sets meet some nondegeneracy requirement.

11.2. Definition. - Let $P \subseteq \mathbb{R}^{n}$ be an $m$-dimensional polyhedral set. For each $x \in P$ we let

$$
\mathscr{W}_{P}(x)=\mathbf{G}(n, m) \cap\left\{W: \mathscr{H}^{m}((x+W) \cap P \cap \mathbf{B}(x, r))>0 \text { for every } r>0\right\} .
$$

We also define

$$
\mathscr{W}_{P}=\cup\left\{\mathscr{W}_{P}(x): x \in P\right\}
$$

(which is a finite subset of $\mathbf{G}(n, m)$ ). We say that $P$ is nondegenerate whenever for every $x \in P$ the following holds. If card $\mathscr{W}_{P}(x) \geqslant 3$ then $\operatorname{dim} \cap \mathscr{W}_{P}(x) \leqslant m-2$.

Notice that if $P$ is a nondegenerate $m$-dimensional polyhedral set then so is each of its relatively open subsets.

For use in the proof we introduce the following definition. In the remainder of this section we let $\mathscr{C}_{k, n}$ denote the collection of dyadic cubes of mesh $2^{-k}$ in $\mathbb{R}^{n}$.

11.3. Definition. - A pseudodyadic cell is a convex set $C \subseteq \mathbb{R}^{n}$ with the property that

$$
C=\cup\left\{D: D \in \mathscr{C}_{k, n} \text { and } D \subseteq C\right\}
$$

for some integer $k \in \mathbb{N}$. The smallest such integer is called the generation of $C$ and denoted by gen $C$.

We are now ready to state the main result of this Part.

\footnotetext{
(14) We insist that the sets $Z_{k}$ appearing in this definition be relatively open. This prevents from having to deal with "boundary points" in applying Theorem 12.3 when we prove the approximation Theorem 6.1. That way, one technical burden is indeed avoided, but the statement and proof of Lemma 14.1 feel somewhat unpleasant — an annoyance reflected in the several cases to be dealt with in the proof of Theorem 14.3. There is no free lunch.
} 


\subsection{Theorem. - Assume that}

(A) $X \subseteq \mathbb{R}^{n}$ is compact and $\left(\mathscr{H}^{n-1}, n-1\right)$ rectifiable;

(B) $\varepsilon>0$.

There then exist an open set $U \supseteq X$, a Lipschitzian map $f: U \rightarrow U$ and a set $P$ such that Clos $P \subseteq U$ verifying the following properties:

(D) $|f(x)-x|_{\infty}<\varepsilon$ for every $x \in U$;

(E) $f(X) \subseteq P$;

(F) $\operatorname{dist}_{\mathscr{H}}(X, P) \leqslant \varepsilon$;

(G) For every $S \subseteq \mathbb{R}^{n}$ and $\eta \geqslant \varepsilon$ one has

$$
\mathscr{H}^{n-1}\left(P \cap \mathbf{U}_{\infty}(S, \eta)\right)<\varepsilon+\mathscr{H}^{n-1}\left(X \cap \mathbf{U}_{\infty}(S, 3 \eta)\right) ;
$$

(H) $\left|\mathscr{H}^{n-1}(X)-\mathscr{H}^{n-1}(P)\right|<\varepsilon$;

(I) $P$ is an $\mathscr{H}^{n-1}$ essential nondegenerate $n$ - 1-dimensional polyhedral set;

(J) $f$ is $\varepsilon \sqrt{n}$-homotopic to the identity of $U$ in the Lipschitzian category.

Proof. - We let $G \subseteq X$ be the set of points $x \in X$ such that there exists $W_{x} \in \mathbf{G}(n, n-1)$ with $^{(15)}$

$$
r^{1-n} \mathbf{T}_{x, r \#}\left(\mathscr { H } ^ { n - 1 } \llcorner X ) \rightarrow \mathscr { H } ^ { n - 1 } \left\llcornerW_{x} \text { as } r \rightarrow 0^{+}\right.\right.
$$

(weakly as Radon measures). Then $\mathscr{H}^{n-1}(X \backslash G)=0$.

We let $\beta>0$ and $\delta>0$ be some constants to be determined later. For each $x \in G$ we choose a decreasing sequence of positive numbers $r_{j}(x) \downarrow 0$ as $j \uparrow \infty$ such that $r_{1}(x) \leqslant \varepsilon / 4$ and

$$
\left(\mathscr{H}^{n-1}\llcorner X)\left(\mathbf{B}_{\infty}\left(x, r_{j}(x)\right) \backslash \mathbf{U}_{\infty}\left(x, r_{j}(x)\right)\right)=0 .\right.
$$

We notice that for every $x \in G$ one has

$$
\begin{aligned}
0 & =\left(\mathscr{H}^{n-1}\left\llcorner W_{x}\right)\left(\mathbf{B}_{\infty}(0,1) \backslash \mathbf{U}_{2}\left(W_{x}, \delta\right)\right)\right. \\
& \geqslant \limsup _{j \rightarrow \infty} r_{j}(x)^{1-n}\left(\mathscr{H}^{n-1}\llcorner X)\left(\mathbf{B}_{\infty}\left(x, r_{j}(x)\right) \backslash \mathbf{U}_{2}\left(x+W_{x}, \delta r_{j}(x)\right)\right) .\right.
\end{aligned}
$$

Therefore there exists an integer $j_{1}(x)$ so that

$$
\left(\mathscr{H}^{n-1}\llcorner X)\left(\mathbf{B}_{\infty}\left(x, r_{j}(x)\right) \backslash \mathbf{U}_{2}\left(x+W_{x}, \delta r_{j}(x)\right)\right)<\beta r_{j}(x)^{n-1}\right.
$$

whenever $j \geqslant j_{1}(x)$.

For $x \in G$ we also notice that

$$
\begin{aligned}
\limsup _{j \rightarrow \infty} r_{j}(x)^{1-n}\left(\mathscr{H}^{n-1}\llcorner X)\right. & \left(\mathbf{B}_{\infty}\left(x, r_{j}(x)\right)\right) \leqslant\left(\mathscr{H}^{n-1}\left\llcorner W_{x}\right)\left(\mathbf{B}_{\infty}(0,1)\right)\right. \\
& =\left(\mathscr{H}^{n-1}\left\llcorner W_{x}\right)\left(\mathbf{U}_{\infty}(0,1)\right)\right. \\
& \leqslant \liminf _{j \rightarrow \infty} r_{j}(x)^{1-n}\left(\mathscr{H}^{n-1}\llcorner X)\left(\mathbf{U}_{\infty}\left(x, r_{j}(x)\right)\right) .\right.
\end{aligned}
$$

Therefore,

$$
\lim _{j \rightarrow \infty} r_{j}(x)^{1-n}\left(\mathscr{H}^{n-1}\llcorner X)\left(\mathbf{U}_{\infty}\left(x, r_{j}(x)\right)\right)=\left(\mathscr{H}^{n-1}\left\llcorner W_{x}\right)\left(\mathbf{U}_{\infty}(0,1)\right) .\right.\right.
$$

$\overline{(15)} \mathbf{T}_{x, r}(y)=(y-x) / r$.

$4^{\mathrm{e}}$ SÉRIE - TOME $42-2009-\mathrm{N}^{\circ} 1$ 
In other words there exists an integer $j_{2}(x)$ such that

$$
\mid\left(\mathscr{H}^{n-1}\llcorner X)\left(\mathbf{U}_{\infty}\left(x, r_{j}(x)\right)\right)-\left(\mathscr{H}^{n-1}\left\llcorner\left(x+W_{x}\right)\right)\left(\mathbf{U}_{\infty}\left(x, r_{j}(x)\right)\right) \mid<\beta r_{j}(x)^{n-1}\right.\right.
$$

whenever $j \geqslant j_{2}(x)$.

Next we infer from the Vitali-Besicovitch covering Theorem ${ }^{(16)}$ and (17) that there are $x_{1}, \ldots, x_{p} \in G$ and $\rho_{i}=r_{j\left(x_{i}\right)}\left(x_{i}\right), i=1, \ldots, p$, with

$$
j\left(x_{i}\right) \geqslant \max \left\{j_{1}\left(x_{i}\right), j_{2}\left(x_{i}\right)\right\},
$$

$i=1, \ldots, p$, the cubes $\mathbf{B}_{\infty}\left(x_{i}, \rho_{i}\right)$ are pairwise disjoint and

$$
\mathscr{H}^{n-1}\left(X \backslash \cup_{i=1}^{p} \mathbf{B}_{\infty}\left(x_{i}, \rho_{i}\right)\right)<\beta .
$$

We claim that for each $i=1, \ldots, p$ there exists a pseudodyadic cell $C_{i} \subseteq \mathbf{U}_{\infty}\left(x_{i}, \rho_{i}\right)$ (recall Definition 11.3) such that on letting $\bar{C}_{i}=\operatorname{Clos} C_{i}$ the following hold

(i) $\left(\mathscr{H}^{n-1}\llcorner X)\left(\mathbf{B}_{\infty}\left(x_{i}, \rho_{i}\right) \backslash C_{i}\right)<\beta / p\right.$;

(ii) $\left(\mathscr{H}^{n-1}\llcorner X)\left(\bar{C}_{i} \backslash \mathbf{U}_{2}\left(x_{i}+W_{x_{i}}, \delta \rho_{i}\right)\right)<\beta \rho_{i}^{n-1}\right.$;

(iii) $\mid\left(\mathscr{H}^{n-1}\llcorner X)\left(C_{i}\right)-\left(\mathscr{H}^{n-1}\left\llcorner\left(x_{i}+W_{x_{i}}\right)\right)\left(\bar{C}_{i}\right) \mid<\beta \rho_{i}^{n-1}\right.\right.$;

(iv) $\mathbf{B}_{2}\left(x_{i}, \rho_{i} / 2\right) \subseteq \bar{C}_{i} \subseteq \mathbf{B}_{2}\left(x_{i}, \sqrt{n} \rho_{i}\right)$.

In order to see this we fix $i=1, \ldots, p$ and we define for every $k=1,2, \ldots$

$$
C_{i, k}=\cup\left\{D: D \in \mathscr{C}_{k, n} \text { and } D \subseteq \mathbf{U}_{\infty}\left(x_{i}, \rho_{i}\right)\right\} .
$$

Notice that the $C_{i, k}$ are bounded convex pseudodyadic cells. Also $C_{i, k} \subseteq C_{i, k+1}$ and

$$
\cup_{k=1}^{\infty} C_{i, k}=\mathbf{U}_{\infty}\left(x_{i}, \rho_{i}\right) .
$$

It immediately follows that

$$
\begin{aligned}
\lim _{k \rightarrow \infty}\left(\mathscr{H}^{n-1}\llcorner X)\left(\mathbf{B}_{\infty}\left(x_{i}, \rho_{i}\right) \backslash C_{i, k}\right)\right. & \\
& =\left(\mathscr{H}^{n-1}\llcorner X)\left(\mathbf{B}_{\infty}\left(x_{i}, \rho_{i}\right) \backslash \mathbf{U}_{\infty}\left(x_{i}, \rho_{i}\right)\right)=0\right.
\end{aligned}
$$

according to (18), so that (i) is verified with $C_{i}=C_{i, k}$ whenever $k$ is large enough. We also infer that for every $k=1,2, \ldots$

$$
\begin{aligned}
\left(\mathscr { H } ^ { n - 1 } \llcorner X ) \left(\bar{C}_{i, k} \backslash\right.\right. & \left.\mathbf{U}_{2}\left(x_{i}+W_{x_{i}}, \delta \rho_{i}\right)\right) \\
& \leqslant\left(\mathscr{H}^{n-1}\llcorner X)\left(\mathbf{B}_{\infty}\left(x_{i}, \rho_{i}\right) \backslash \mathbf{U}_{2}\left(x_{i}+W_{x_{i}}, \delta \rho_{i}\right)\right)<\beta \rho_{i}^{n-1},\right.
\end{aligned}
$$

according to (19). This shows that (ii) holds for $C_{i}=C_{i, k}$. On the other hand,

$$
\lim _{k \rightarrow \infty}\left(\mathscr{H}^{n-1}\llcorner X)\left(C_{i, k}\right)=\left(\mathscr{H}^{n-1}\llcorner X)\left(\mathbf{U}_{\infty}\left(x_{i}, \rho_{i}\right)\right)\right.\right.
$$

and

$$
\lim _{k \rightarrow \infty}\left(\mathscr{H}^{n-1}\left\llcorner\left(x_{i}+W_{x_{i}}\right)\right)\left(C_{i, k}\right)=\left(\mathscr{H}^{n-1}\left\llcorner\left(x_{i}+W_{x_{i}}\right)\right)\left(\mathbf{U}_{\infty}\left(x_{i}, \rho_{i}\right)\right) .\right.\right.
$$

We then infer from (20) that (iii) holds for $C_{i}=C_{i, k}$ if $k$ is sufficiently large. Condition (iv) trivially holds for large $k$ as well. This completes the proof of our claim.

(16) Here the Vitali-Besicovitch covering Theorem is applied to a family of balls with respect to the maximum norm in $\mathbb{R}^{n}$, see [11, 2.8.9 and 2.8.15] 
We let $g=\max \left\{\right.$ gen $C_{1}, \ldots$, gen $\left.C_{p}\right\}$. Notice that $2^{-g} \leqslant \operatorname{diam}_{\infty} C_{1} \leqslant 2 \rho_{1} \leqslant \varepsilon / 2$. There are finitely many dyadic cubes of generation $g, D_{1}, \ldots, D_{q}$, which are disjoint from $\cup_{i=1}^{p} C_{i}$ and are such that

$$
X \subseteq\left(\cup_{i=1}^{p} \bar{C}_{i}\right) \cup\left(\cup_{j=1}^{q} D_{j}\right)
$$

and $D_{j} \cap X \neq \varnothing$ for each $j=1, \ldots, q$.

We now explain how we possibly need to rotate slightly the $W_{x_{i}}, i=1, \ldots, p$, in order to meet the nondegeneracy requirement of the approximating piecewise flat set (to be defined later on). For each $i=1, \ldots, p$ and $l=1, \ldots, 2 n$ we let $A_{i, l}$ denote the $n-1$ dimensional affine subspaces of $\mathbb{R}^{n}$ characterized by the relation Bdry $C_{i} \subseteq \cup_{l=1}^{2 n} A_{i, l}$. We define similarly $B_{j, l}, j=1, \ldots, q, l=1, \ldots, 2 n$, by the requirement that Bdry $D_{j} \subseteq \cup_{l=1}^{2 n} B_{j, l}$. Next we put

$$
A_{i, l}^{\prime}=A_{i, l} \cap \mathbf{U}\left(A_{i, l} \cap \operatorname{Bdry} C_{i}, 2^{-g-3}\right)
$$

and

$$
B_{j, l}^{\prime}=B_{j, l} \cap \mathbf{U}\left(B_{j, l} \cap \text { Bdry } D_{j}, 2^{-g-3}\right),
$$

$i=1, \ldots, p, j=1, \ldots, q, l=1, \ldots, 2 n$. We will define, inductively on $i=0,1, \ldots, p$, families $\mathscr{F}_{i}$ of subsets of $\mathbb{R}^{n}$ such that $\cup \mathscr{F}_{i}$ is a nondegenerate $n-1$ dimensional piecewise flat set. We start with

$$
\mathscr{F}_{0}=\left\{A_{i, l}^{\prime}: i=1, \ldots, p, l=1, \ldots, 2 n\right\} \cup\left\{B_{j, l}^{\prime}: j=1, \ldots, q, l=1, \ldots, 2 n\right\}
$$

and we readily check that $\cup \mathscr{F}_{0}$ is nondegenerate. Our induction hypothesis about $\mathscr{F}_{i}$ will also include that $\operatorname{dist}\left(S, x_{i^{\prime}}\right)>0$ for each $S \in \mathscr{F}_{i}$ and each $i^{\prime}=i+1, \ldots, p$ (notice that this holds for $i=0$ ). Given $i=1, \ldots, p$ and assuming that $\mathscr{F}_{i-1}$ has already been defined we notice that

$$
\begin{aligned}
\mathscr{B}_{i}=\mathbf{G}(n, n-1) & \cap\left\{W: \text { for some distinct } S, S^{\prime} \in \mathscr{F}_{i},\right. \\
& \left.\left(x_{i}+W\right) \cap S \cap S^{\prime} \text { generates an } n-2 \text {-dimensional affine subspace }\right\}
\end{aligned}
$$

is a finite set. Therefore there exists $W_{x_{i}}^{\prime} \in \mathbf{G}(n, n-1) \backslash \mathscr{B}_{i}$ as close as we wish to $W_{x_{i}}$, in particular we can assume that estimates (ii) and (iii) above hold with $W_{x_{i}}$ replaced by $W_{x_{i}}^{\prime}$. Finally we can choose $0<\delta_{i}<\varepsilon / 2$ such that

$$
\left(\mathscr{H}^{n-1}\left\llcorner\left(x_{i}+W_{x_{i}}^{\prime}\right)\right)\left(\mathbf{B}_{\infty}\left(C_{i}, \delta_{i}\right)\right) \leqslant\left(\mathscr{H}^{n-1}\left\llcorner\left(x_{i}+W_{x_{i}}^{\prime}\right)\right)\left(\bar{C}_{i}\right)+\beta / p\right.\right.
$$

and also such that

$$
\mathscr{F}_{i}=\mathscr{F}_{i-1} \cup\left\{\left(x_{i}+W_{x_{i}}^{\prime}\right) \cap \mathbf{U}_{\infty}\left(C_{i}, \delta_{i}\right)\right\}
$$

has the property that $\cup \mathscr{F}_{i}$ is nondegenerate (because $W_{x_{i}}^{\prime} \notin \mathscr{B}_{i}$ ) and $\mathscr{F}_{i}$ verifies our induction hypothesis.

We now turn to preparing for the definitions of $f$ and $P(P$ will be a relatively open subset of $\mathscr{F}_{p}$ ). For each $i=1, \ldots, p$ we let $C_{i}^{-}$and $C_{i}^{+}$denote the closure of the two components of $C_{i} \backslash\left(x_{i}+W_{x_{i}}^{\prime}\right)$. We observe that there exist $a_{i}^{ \pm} \in C_{i}^{ \pm}$such that

$$
\mathbf{B}\left(a_{i}^{ \pm}, \rho_{i} / 8\right) \subseteq \operatorname{Int} C_{i}^{ \pm} \subseteq \mathbf{B}\left(a_{i}^{ \pm},(1+\sqrt{n}) \rho_{i}\right)
$$

and

$$
\mathbf{B}\left(a_{i}^{ \pm}, \rho_{i} / 8\right) \cap \mathbf{U}\left(\left(x_{i}+W_{x_{i}}^{\prime}\right), \delta \rho_{i}\right)=\varnothing
$$


as follows from (iv) above, and provided we assume that $\delta<1 / 4$. Define

$$
E_{i}^{ \pm}=X \cap \operatorname{Int} C_{i}^{ \pm} \backslash \mathbf{U}\left(x_{i}+W_{x_{i}}^{\prime}, \delta \rho_{i}\right) .
$$

According to Proposition 9.7 there exist $b_{i}^{ \pm} \in \mathbf{B}\left(a_{i}^{ \pm}, \rho_{i} / 8\right) \backslash E_{i}^{ \pm}$such that

$$
\mathscr{H}^{n-1}\left(\pi_{C_{i}^{ \pm}, b_{i}^{ \pm}}\left(E_{i}^{ \pm}\right)\right) \leqslant C_{1}(n) \mathscr{H}^{n-1}\left(E_{i}^{ \pm}\right)
$$

where

$$
C_{1}(n)=n 2^{6 n-1}(1+\sqrt{n})^{2 n-1} .
$$

We choose $\mathscr{H}^{n-1}$ essential relatively open sets

$$
E_{i}^{\prime} \subseteq \cup_{l=1}^{2 n} A_{i, l}^{\prime} \subseteq \cup \mathscr{F}_{p}
$$

such that

$$
\pi_{C_{i}^{-}, b_{i}^{-}}\left(E_{i}^{-}\right) \cup \pi_{C_{i}^{+}, b_{i}^{+}}\left(E_{i}^{+}\right) \subseteq E_{i}^{\prime} \subseteq \cup \mathscr{F}_{p}
$$

and

$$
\mathscr{H}^{n-1}\left(E_{i}^{\prime}\right) \leqslant C_{1}(n) \mathscr{H}^{n-1}\left(E_{i}^{-} \cup E_{i}^{+}\right)+\beta / p .
$$

It follows from (23) and $b_{i}^{ \pm} \in \mathbf{B}\left(a_{i}^{ \pm}, \rho_{i} / 8\right)$ that

$$
\pi_{C_{i}^{ \pm}, b_{i}^{ \pm}}\left(C_{i}^{ \pm} \cap \mathbf{U}\left(x_{i}+W_{x_{i}}^{\prime}, \delta \rho_{i}\right)\right) \subseteq \mathbf{U}\left(x_{i}+W_{x_{i}}^{\prime}, \delta \rho_{i}\right) \cap \operatorname{Bdry} C_{i},
$$

and we observe that

(27)

$$
\begin{aligned}
\mathscr{H}^{n-1}\left(\mathbf{U}\left(x_{i}+W_{x_{i}}^{\prime}, \delta \rho_{i}\right)\right. & \left.\cap \operatorname{Bdry} C_{i}\right) \\
& \leqslant \mathscr{H}^{n-1}\left(\mathbf{U}\left(x_{i}+W_{x_{i}}^{\prime}, \delta \rho_{i}\right) \cap \operatorname{Bdry} \mathbf{B}_{\infty}\left(x_{i}, \rho_{i}\right)\right) \leqslant C_{2}(n) \delta \rho_{i}^{n-1},
\end{aligned}
$$

where

We also define

$$
C_{2}(n)=n 2^{n / 2}
$$

$$
\begin{aligned}
& \qquad E_{\mathrm{b}}=X \cap\left(\cup_{i=1}^{p}\left(\operatorname{Bdry} C_{i}\right) \backslash \mathbf{U}\left(x_{i}+W_{x_{i}}^{\prime}, \delta \rho_{i}\right)\right) \\
& \text { and we choose an } \mathscr{H}^{n-1} \text { essential relatively open set }
\end{aligned}
$$

$$
E_{b}^{\prime} \subseteq \cup_{i=1}^{p} \cup_{l=1}^{2 n} A_{i, l}^{\prime}
$$

such that

$$
E_{\mathrm{b}} \subseteq E_{b}^{\prime}
$$

and

$$
\mathscr{H}^{n-1}\left(E_{\mathrm{b}}^{\prime}\right) \leqslant \mathscr{H}^{n-1}\left(E_{\mathrm{b}}\right)+\beta .
$$

Notice that

$$
\mathscr{H}^{n-1}\left(E_{b}\right) \leqslant \sum_{i=1}^{p} \mathscr{H}^{n-1}\left(X \cap \bar{C}_{i} \backslash \mathbf{U}\left(x_{i}+W_{x_{i}}^{\prime}, \delta \rho_{i}\right)\right) \leqslant \beta \sum_{i=1}^{p} \rho_{i}^{n-1}
$$

according to (ii).

Similarly, referring to Proposition 9.7, for each $j=1, \ldots, q$, we find $c_{j} \in \operatorname{Int} D_{j} \backslash X$ such that

$$
\mathscr{H}^{n-1}\left(\pi_{D_{j}, c_{j}}\left(F_{j}\right)\right) \leqslant C_{3}(n) \mathscr{H}^{n-1}\left(F_{j}\right)
$$


where

$$
F_{j}=X \cap D_{j}
$$

and

$$
C_{3}(n)=4 n^{n+1 / 2} .
$$

We choose $\mathscr{H}^{n-1}$ essential relatively open sets

$$
F_{j}^{\prime} \subseteq \cup_{l=1}^{2 n} B_{j, l}^{\prime}
$$

such that

$$
\pi_{D_{j}, c_{j}}\left(F_{j}\right) \subseteq F_{j}^{\prime}
$$

and

$$
\mathscr{H}^{n-1}\left(F_{j}^{\prime}\right) \leqslant C_{3}(n) \mathscr{H}^{n-1}\left(F_{j}\right)+\beta / q .
$$

We are now ready to define $P$.

$$
\begin{aligned}
P= & \left(\cup_{i=1}^{p}\left(x_{i}+W_{x_{i}}^{\prime}\right) \cap \mathbf{U}\left(C_{i}, \delta_{i}\right)\right) \\
& \cup\left(\cup_{i=1}^{p} \mathbf{U}\left(x_{i}+W_{x_{i}}^{\prime}\right) \cap \operatorname{Bdry} C_{i}\right) \cup\left(\cup_{i=1}^{p} E_{i}^{\prime}\right) \cup E_{b}^{\prime} \cup\left(\cup_{j=1}^{q} F_{j}^{\prime}\right) .
\end{aligned}
$$

As $P \subseteq \cup \mathscr{F}_{p}$ is relatively open and $\cup \mathscr{F}_{p}$ is a nondegenerate $n-1$ dimensional polyhedral set, we conclude $P$ has the same property. Furthermore all the sets arising in the definition of $P$ are $\mathscr{H}^{n-1}$ essential, so that $P$ is as well. We now turn to proving that

$$
\operatorname{dist}_{\mathscr{H}}(X, P) \leqslant \varepsilon .
$$

Each $\xi \in \cup \mathscr{F}_{p}$ is $\varepsilon / 2$ close (in the norm $|\cdot|_{\infty}$ ) to either some $C_{i}$ or some $D_{j}$, each of which meets $X$ nontrivially and has diameter less than $\varepsilon / 2$. Therefore

$$
P \subseteq \cup \mathscr{F}_{p} \subseteq \mathbf{B}_{\infty}(X, \varepsilon) .
$$

Furthermore if $\xi \in X$ then either $\xi \in \bar{C}_{i}$ for some $i=1, \ldots, p$, whence $\left|\xi-x_{i}\right|_{\infty} \leqslant \rho_{i} \leqslant \varepsilon / 4$, or $\xi \in D_{j}$ for some $j=1, \ldots, q$, and then $\left|\pi_{D_{j}, c_{j}}(\xi)-\xi\right|_{\infty} \leqslant \operatorname{diam}_{\infty} D_{j} \leqslant \varepsilon$. As $x_{i} \in P$ and $\operatorname{im} \pi_{D_{j}, c_{j}} \uparrow X \cap D_{j} \subseteq P$ we conclude that

$$
X \subseteq \mathbf{B}_{\infty}(P, \varepsilon) .
$$

This completes the proof of (33).

Prior to estimating the measure of $P$ we observe that

$$
\begin{aligned}
2 \sum_{i=1}^{p} \rho_{i}^{n-1} & \leqslant \sum_{i=1}^{p}\left(2 \rho_{i}\right)^{n-1}=\sum_{i=1}^{p} \mathscr{H}^{n-1}\left(\left(x_{i}+\operatorname{span}\left\{e_{1}, \ldots, e_{n-1}\right\}\right) \cap \mathbf{B}_{\infty}\left(x_{i}, \rho_{i}\right)\right) \\
& \leqslant \sum_{i=1}^{p} \mathscr{H}^{n-1}\left(\left(x_{i}+W_{x_{i}}\right) \cap \mathbf{B}_{\infty}\left(x_{i}, \rho_{i}\right)\right)
\end{aligned}
$$

which, according to (20), is bounded by

$$
\leqslant \sum_{i=1}^{p}\left(\beta \rho_{i}^{n-1}+\mathscr{H}^{n-1}\left(X \cap \mathbf{B}_{\infty}\left(x_{i}, \rho_{i}\right)\right)\right) \leqslant \beta \sum_{i=1}^{p} \rho_{i}^{n-1}+\mathscr{H}^{n-1}(X) .
$$

$4^{\mathrm{e}}$ SÉRIE - TOME $42-2009-\mathrm{N}^{\circ} 1$ 
If $\beta \leqslant 1-$ as we will subsequently assume - , then

$$
\sum_{i=1}^{p} \rho_{i}^{n-1} \leqslant \mathscr{H}^{n-1}(X) .
$$

We are ready to prove (locally, at a scale not too small) an upper estimate for the measure of $P$. Let $S \subseteq \mathbb{R}^{n}$ and $\eta \geqslant \varepsilon$ and define

$$
I_{S}=\{1, \ldots, p\} \cap\left\{i: \mathbf{U}_{\infty}(S, \eta) \cap\left(x_{i}+W_{x_{i}}^{\prime}\right) \cap \mathbf{U}_{\infty}\left(C_{i}, \delta_{i}\right) \neq \varnothing\right\} .
$$

Notice that if $i \in I_{S}$ and $\xi \in X \cap \mathbf{B}_{\infty}\left(x_{i}, \rho_{i}\right)$ then $\xi \in \mathbf{U}_{\infty}(S, 3 \eta)$. $\mathbf{P}$ Indeed choose $\zeta \in$ $\mathbf{U}_{\infty}(S, \eta) \cap\left(x_{i}+W_{x_{i}}^{\prime}\right) \cap \mathbf{U}_{\infty}\left(C_{i}, \delta_{i}\right)$, so that $\left|\zeta-x_{i}\right|_{\infty} \leqslant \rho_{i}+\delta_{i}<\varepsilon$, and in turn choose $\zeta^{\prime} \in S$ such that $\left|\zeta-\zeta^{\prime}\right|_{\infty}<\eta$. Then $\left|\xi-\zeta^{\prime}\right|_{\infty} \leqslant\left|\xi-x_{i}\right|_{\infty}+\left|x_{i}-\zeta\right|_{\infty}+\left|\zeta-\zeta^{\prime}\right|_{\infty}<\rho_{i}+\varepsilon+\eta \leqslant 3 \eta$.

Q Therefore

$$
\begin{aligned}
\mathscr{H}^{n-1}\left(P \cap \mathbf{U}_{\infty}(S, \eta)\right) \leqslant & \sum_{i \in I_{S}} \mathscr{H}^{n-1}\left(\left(x_{i}+W_{x_{i}}^{\prime}\right) \cap \mathbf{U}_{\infty}\left(C_{i}, \delta_{i}\right)\right) \\
& +\sum_{i=1}^{p} \mathscr{H}^{n-1}\left(\mathbf{U}\left(x_{i}+W_{x_{i}}^{\prime}, \delta \rho_{i}\right) \cap \operatorname{Bdry} C_{i}\right) \\
& +\sum_{i=1}^{p} \mathscr{H}^{n-1}\left(E_{i}^{\prime}\right) \\
& +\mathscr{H}^{n-1}\left(E_{b}^{\prime}\right) \\
& +\sum_{j=1}^{q} \mathscr{H}^{n-1}\left(F_{j}^{\prime}\right) \\
\leqslant & \sum_{i \in I_{S}}\left(\mathscr{H}^{n-1}\left(X \cap \mathbf{B} \infty\left(x_{i}, \rho_{i}\right)\right)+\beta \rho_{i}^{n-1}+2 \beta / p\right) \\
& +\sum_{i=1}^{p} C_{2}(n) \delta \rho_{i}^{n-1} \\
& +\sum_{i=1}^{p}\left(C_{1}(n) \beta \rho_{i}^{n-1}+\beta / p\right) \\
& +\beta\left(1+\sum_{i=1}^{p} \rho_{i}^{n-1}\right) \\
& +\beta\left(C_{3}(n)+1\right) \\
\leqslant & \mathscr{H}^{n-1}\left(X \cap \mathbf{U}_{\infty}(S, 3 \eta)\right) \\
& +\beta\left(5+C_{3}(n)+\left(2+C_{1}(n)\right) \mathscr{H}^{n-1}(X)\right) \\
& +\delta C_{2}(n) \mathscr{H}^{n-1}(X),
\end{aligned}
$$

where we have used the following inequalities in order to estimate each of the five terms in the above sum: (22), (i) and (iii) for the first term; (27) for the second term; (25) and (ii) for the third term; (29) and (30) for the fourth term; and (32) and (21) for the fifth term. Choosing $\beta$ and $\delta$ small enough (depending upon $\varepsilon, C_{1}(n), C_{2}(n), C_{3}(n)$ and $\mathscr{H}^{n-1}(X)$ ) we can readily 
achieve the following inequality

$$
\mathscr{H}^{n-1}\left(P \cap \mathbf{U}_{\infty}(S, \eta)\right)<\varepsilon+\mathscr{H}^{n-1}\left(X \cap \mathbf{U}_{\infty}(S, 3 \eta)\right),
$$

(which is conclusion $(\mathrm{G})$ ), in particular

$$
\mathscr{H}^{n-1}(P) \leqslant \mathscr{H}^{n-1}(X)+\varepsilon .
$$

On the other hand,

$$
\mathscr{H}^{n-1}(P) \geqslant \sum_{i=1}^{p} \mathscr{H}^{n-1}\left(\left(x_{i}+W_{x_{i}}^{\prime}\right) \cap \bar{C}_{i}\right)
$$

which, according to (iii), (i) and (34), is bounded below by

$$
\begin{aligned}
& \geqslant \sum_{i=1}^{p} \mathscr{H}^{n-1}\left(X \cap \mathbf{B}_{\infty}\left(x_{i}, \rho_{i}\right)\right)-\beta\left(1+\mathscr{H}^{n-1}(X)\right) \\
& \geqslant \mathscr{H}^{n-1}(X)-\beta\left(2+\mathscr{H}^{n-1}(X)\right),
\end{aligned}
$$

where the last inequality follows from (21). We obtain that

$$
\mathscr{H}^{n-1}(P) \geqslant \mathscr{H}^{n-1}(X)-\varepsilon
$$

provided $\beta$ is small enough.

In order that the proof of the theorem be complete it remains to define $U$ and $f$ so that they verify the stated properties. For each $i=1, \ldots, p$ we choose $\sigma_{i}^{ \pm}>0$ such that

$$
\mathbf{B}\left(b_{i}^{ \pm}, \sigma_{i}^{ \pm}\right) \cap X=\varnothing \text { and } \mathbf{B}\left(b_{i}^{ \pm}, \sigma_{i}^{ \pm}\right) \subseteq \operatorname{Int} C_{i}^{ \pm},
$$

and for each $j=1, \ldots, q$ we choose $\tau_{j}>0$ such that

$$
\mathbf{B}\left(c_{j}, \tau_{j}\right) \cap X=\varnothing \text { and } \mathbf{B}\left(c_{j}, \tau_{j}\right) \subseteq \operatorname{Int} D_{j} .
$$

We put

$$
U=\mathbb{R}^{n} \backslash\left[\left(\cup_{i=1}^{p} \mathbf{B}\left(b_{i}^{-}, \sigma_{i}^{-}\right)\right) \cup\left(\cup_{i=1}^{p} \mathbf{B}\left(b_{i}^{+}, \sigma_{i}^{+}\right)\right) \cup\left(\cup_{j=1}^{q} \mathbf{B}\left(c_{j}, \tau_{j}\right)\right)\right]
$$

and we define $f$ as follows.

$$
\begin{aligned}
& f \uparrow \bar{C}_{i}^{ \pm} \backslash \mathbf{B}\left(b_{i}^{ \pm}, \sigma_{i}^{ \pm}\right)=\pi_{\bar{C}_{i}^{ \pm}, b_{i}^{ \pm}}, i=1, \ldots, p \\
& f \uparrow \bar{D}_{j} \backslash \mathbf{B}\left(c_{j}, \tau_{j}\right)=\pi_{\bar{D}_{j}, c_{j}}, j=1, \ldots, q \\
& f \uparrow \mathbb{R}^{n} \backslash\left(\left(\cup_{i=1}^{p} \bar{C}_{i}\right) \cup\left(\cup_{j=1}^{q} \bar{D}_{j}\right)\right)=\mathrm{id}_{\mathbb{R}^{n} \backslash\left(\left(\cup_{i=1}^{p} \bar{C}_{i}\right) \cup\left(\cup_{j=1}^{q} \bar{D}_{j}\right)\right)} .
\end{aligned}
$$

It is then immediate from the definition of $P$ that $f(X) \subseteq P$. Notice also that im $f \subseteq U$. Since $\operatorname{diam}_{\infty} C_{i}<\varepsilon, i=1, \ldots, p$, and $\operatorname{diam}_{\infty} D_{j}<\varepsilon, j=1, \ldots, q$, we readily infer that $|f(x)-x|_{\infty}<\varepsilon, x \in U$. Finally, that $f$ is Lipschitzian and $\varepsilon \sqrt{n}$-homotopic to $\operatorname{id}_{U}$ in the Lipschitzian category is an application of Lemma 10.3 .

$4^{\text {e }}$ SÉRIE - TOME $42-2009-\mathrm{N}^{\mathrm{o}} 1$ 


\section{PART III}

\section{APPROXIMATION: THE BOUNDARY CASE}

\section{Interpolating a particular retraction on the boundary}

We start by stating a version of the standard "tubular neighborhood theorem".

12.1. Theorem. - Let $B \subseteq \mathbb{R}^{3}$ be a 1-dimensional compact submanifold of class $C^{k}$, $k=2,3, \ldots$ There exists $R>0$ with the following properties.

(A) For every $x \in \mathbf{U}(B, R)$ there exists a unique $\xi \in B$ such that $|\xi-x|=\operatorname{dist}(x, B)$ and this $\xi$ is denoted by $\pi(x)$;

(B) The map $\pi: \mathbf{U}(B, R) \rightarrow B$ is Lipschitzian and of class $C^{k-1}$;

(C) The map $d: \mathbf{U}(B, R) \rightarrow \mathbb{R}: x \mapsto \operatorname{dist}(x, B)$ is of class $C^{k}$;

(D) For every $\xi \in B$, letting $W(\xi)=\left(T_{\xi} B\right)^{\perp} \in \mathbf{G}(3,2)$ we have that $\pi^{-1}\{\xi\}=(\xi+$ $W(\xi)) \cap \mathbf{U}(\xi, R)$.

(E) For every $0<r<R$ the set $\mathbb{R}^{3} \cap\{x: \operatorname{dist}(x, B)=r\}$ is a 2-dimensional submanifold of class $C^{k}$ of $\mathbb{R}^{3}$.

12.2. REMARK. - The following comments are in order.

(1) The existence of $R>0$ such that conclusion (A) holds is, by definition, saying that $B$ has positive reach. For that purpose the mere assumption that $B$ be of class $C^{1,1}$ is sufficient, see [10,4.12]. In case $B$ is only of class $C^{1}$ it does not need to have positive reach, as shown by the example $B=\mathbb{R}^{3} \cap\left\{x: x_{2}=\left|x_{1}\right|^{3 / 2}\right.$ and $\left.x_{3}=0\right\}$. Notwithstanding there still exists a map $\pi$ defined in a neighborhood of $B$ which is an "approximate nearest point projection" of class $C^{1}$ and having flat level sets (as stated in conclusion (D) above), see [28, Chap. IV Theorem 10A].

(2) Conclusion (B) follows from straightforward computations; an expression for $D \pi$ showing that $\left\|D \pi(x)-P_{T_{\pi(x)} B}\right\|=O(\operatorname{dist}(x, B))$ can be found for instance in [1, 2.2(2)].

(3) The fact that $d(x)=\operatorname{dist}(x, B)=|x-\pi(x)|, x \in \mathbf{U}(B, R) \backslash B$, has one more degree of differentiability (with respect to $x$ ) than $\pi$ itself can be seen for instance (following R.L. Foote, [16]) on noticing that $\nabla d^{2}(x)=2(x-\pi(x))$.

(4) Conclusion (D) is a consequence of (A) and $x-\pi(x) \in\left(T_{\pi(x)} B\right)^{\perp}$ (which follows from a simple variational argument). Notice (D) implies $W(\pi(x))=\operatorname{ker} D \pi(x)$, a fact that will be used repeatedly without reference.

(5) It readily follows from (D) that each $0<r<R$ is a regular value of $d$, so that (E) holds.

In the remaining part of this section we will use the symbols $B, \pi, d$ and $W$ with the meaning given in Theorem 12.1. We will also abbreviate

$$
V_{r}=\mathbb{R}^{3} \cap\{x: \operatorname{dist}(x, B)<r\}, 0<r \leqslant R,
$$

as well as

$$
M_{r}=\mathbb{R}^{3} \cap\{x: \operatorname{dist}(x, B)=r\}, 0<r<R .
$$

One easily checks that $M_{r}=\operatorname{Bdry} V_{r}$. 
12.3. Theorem. - Assume that $B, \pi, d, V_{r}$ and $M_{r}$ are as above. There then exists $\Lambda>0$ with the following property. For every $0<r<R / 4$ there exists a Lipschitzian map $g_{r}: \mathbb{R}^{3} \rightarrow \mathbb{R}^{3}$ such that the following hold.

(A) $g_{r}(x)=\pi(x)$ for every $x \in \operatorname{Clos} V_{r}, g_{r}(x)=x$ for every $x \in \mathbb{R}^{3} \backslash V_{2 r}$, and $\left|x-g_{r}(x)\right|<2 r$ for every $x \in \mathbb{R}^{3}$

(B) $\operatorname{Lip} g_{r} \leqslant \Lambda$;

(C) The restriction $g_{r}: \mathbb{R}^{3} \backslash$ Clos $V_{r} \rightarrow \mathbb{R}^{3} \backslash B$ is a $C^{1}$ diffeomorphism;

(D) Suppose that $x_{0} \in M_{r}, W_{0} \in \mathbf{G}(3,2)$ and

(D1) $\operatorname{dim} W_{0} \cap T_{x_{0}} M_{r}=1$;

(D2) $W_{0} \cap T_{x_{0}} M_{r} \cap W\left(\pi\left(x_{0}\right)\right)=\{0\}$;

There then exists $\rho_{0}>0$ such that the restriction of $g_{r}$ to $\left(x_{0}+W_{0}\right) \cap \mathbf{U}\left(x_{0}, \rho_{0}\right) \backslash V_{r}$ is a bi-Lipschitzian homeomorphism onto its image.

Proof. - Let $0<r<R / 4$. We choose a function $\lambda_{r}:(0, \infty) \rightarrow(0, \infty)$ with the following properties.

(a) $\lambda_{r}$ is of class $C^{\infty}$ on $(r, \infty)$;

(b) $\lambda_{r}(\rho)=0$ if $0<\rho \leqslant r$;

(c) $\lambda_{r}(\rho)=\rho-r$ if $r \leqslant \rho \leqslant 3 r / 2$;

(d) $\lambda_{r}(\rho)=\rho$ if $2 r \leqslant \rho$;

(e) $\lambda_{r}$ is Lipschitzian and $\operatorname{Lip} \lambda_{r} \leqslant 4$;

(f) $\lambda_{r}(\rho) \leqslant \rho$ for every $\rho>0$;

(g) $\lambda_{r}^{\prime}(\rho) \geqslant 1$ for every $r<\rho<2 r$.

We define $g_{r}: \mathbb{R}^{3} \rightarrow \mathbb{R}^{3}$ by the formula

$$
g_{r}(x)= \begin{cases}x & \text { if } x \in B \cup \mathbb{R}^{3} \backslash V_{2 r} \\ \pi(x)+\lambda_{r}(d(x)) \frac{x-\pi(x)}{d(x)} & \text { if } x \notin B \cup \mathbb{R}^{3} \backslash V_{2 r}\end{cases}
$$

Conclusion (A) is an immediate consequence of the definition and property (b) of $\lambda_{r}$. Notice that $g_{r}$ is of class $C^{1}$ on $\mathbb{R}^{3} \backslash$ Clos $V_{r}$ (the formula defining $g_{r}$ in $V_{2 r} \backslash B$ also coincides with $g_{r}$ on the whole $V_{R} \backslash B$ according to property (d) of $\lambda_{r}$ ). Moreover for $x \in V_{R} \backslash$ Clos $V_{r}$ and $h \in \mathbb{R}^{3}$ one checks that

$$
\begin{aligned}
D g_{r}(x)(h)= & D \pi(x)(h) \\
& +\lambda_{r}^{\prime}(d(x))\langle\nabla d(x), h\rangle\left(\frac{x-\pi(x)}{d(x)}\right) \\
& +\frac{\lambda_{r}(d(x))}{d(x)}\left(h-D \pi(x)(h)-\left(\frac{x-\pi(x)}{d(x)}\right)\langle\nabla d(x), h\rangle\right) .
\end{aligned}
$$

We also recall that Lip $d \leqslant 1$ (and therefore $|\nabla d(x)| \leqslant 1$ for every $x \in V_{R} \backslash B$ ). Referring to (35) as well as properties (e) and (f) of $\lambda_{r}$ we infer that

$$
\left\|D g_{r}(x)\right\| \leqslant \operatorname{Lip} \pi+4+(1+\operatorname{Lip} \pi+1)
$$

for every $x \in V_{R} \backslash B$. We also notice that the pointwise Lipschitz constant of $g_{r}$ at points of $\mathbb{R}^{3} \backslash\left(V_{R} \backslash B\right)$ is bounded by $\max \{1, \operatorname{Lip} \pi\}$. It readily follows that $g_{r}$ is Lipschitzian on $\mathbb{R}^{3}$ with $\operatorname{Lip} g_{r} \leqslant 6+2 \operatorname{Lip} \pi=: \Lambda$. Conclusion (B) follows. 
In order to prove conclusion (C) we observe that the restriction

$$
g_{r}: \mathbb{R}^{3} \backslash \text { Clos } V_{r} \rightarrow \mathbb{R}^{3} \backslash B
$$

is bijective, thus it is sufficient to show that it is locally a $C^{1}$ diffeomorphism. If $d(x)>2 r$ then $g_{r}=\mathrm{id}_{\mathbb{R}^{3}}$ in a neighborhood of $x$. If $d(x)=2 r$ then $D g_{r}(x)=\mathrm{id}_{\mathbb{R}^{3}}$ according to (35) together with properties (a) and (d) of $\lambda_{r}$, so that $g_{r}$ is a $C^{1}$ diffeomorphism of a neighborhood of $x$ onto its image. The case $0<d(x)<2 r$ is dealt with by showing that in fact the restriction

$$
g_{r}: V_{2 r} \backslash \operatorname{Clos} V_{r} \rightarrow V_{2 r} \backslash B
$$

is a $C^{1}$ diffeomorphism. Indeed, letting $\mu_{r}$ denote the inverse of $\lambda_{r}:(r, 2 r) \rightarrow(0,2 r)$ (recall property $(\mathrm{g})$ of $\lambda_{r}$ ) we define

$$
h_{r}: V_{2 r} \backslash B \rightarrow V_{2 r} \backslash \operatorname{Clos} V_{r}
$$

by the formula

$$
h_{r}(y)=\pi(y)+\mu_{r}(d(y)) \frac{y-\pi(y)}{d(y)} .
$$

It is obvious that $h_{r}$ is of class $C^{1}$ and it is a simple matter to verify explicitly that $h_{r}$ is the inverse of $g_{r}$.

It remains to address conclusion (D). We have already established that $g_{r}$ is Lipschitzian. Therefore the proof will be completed upon showing that there are $\rho_{0}>0$ and $\eta>0$ such that

$$
\left|g_{r}(x)-g_{r}(y)\right| \geqslant \eta|x-y|
$$

for every $x, y \in\left(x_{0}+W_{0}\right) \cap \mathbf{U}\left(x_{0}, \rho_{0}\right) \backslash V_{r}$.

For each $x \in \mathbf{U}(B, R) \backslash B$ we abbreviate

$$
n(x)=\frac{x-\pi(x)}{|x-\pi(x)|} .
$$

Notice that $n(x) \in\left(T_{x} M_{d(x)}\right)^{\perp}$ in view of Remark 12.2(3). Since $x-\pi(x) \in W(\pi(x))$ we also infer that $n(x) \in W(\pi(x))$, so that $n(x)$ is the unit exterior normal to the circle $M_{d(x)} \cap(\pi(x)+W(\pi(x))) \cap \mathbf{U}(\pi(x), R)$, at the point $x$, in the affine plane $\pi(x)+W(\pi(x))$.

Claim \#1. There exist $\rho_{1}>0, \beta_{0}>0, \theta_{0}>0$ and a unit vector $v_{0} \in \mathbb{R}^{3}$ with the following properties. For every $x \in \mathbf{U}\left(x_{0}, \rho_{1}\right)$ one has

$$
\begin{gathered}
\operatorname{dim} W_{0} \cap W(\pi(x))=1 \\
\operatorname{dim} W_{0} \cap T_{x} M_{d(x)}=1 \\
\operatorname{dim} W_{0} \cap W(\pi(x)) \cap T_{x} M_{d(x)}=0 .
\end{gathered}
$$

Furthermore if we let $u(x)$ be a unit vector spanning $W_{0} \cap W(\pi(x))$ such that $\langle n(x), u(x)\rangle \geqslant 0$, $0 \leqslant \theta(x) \leqslant \pi / 2$ be the angle between $W_{0} \cap W(\pi(x))$ and $T_{x} M_{d(x)}$, and $e(x)$ be a unit vector spanning $W_{0} \cap T_{x} M_{d(x)}$ then the following hold.

$$
\begin{gathered}
\theta_{0} \leqslant \theta(x) \\
\left\langle v_{0}, D \pi(x)(e(x))\right\rangle \geqslant \beta_{0} \\
\left|P_{W(\pi(x))}\left(v_{0}\right)\right| \leqslant \frac{\beta_{0}^{2}}{64 \Lambda^{2}} .
\end{gathered}
$$


P Regarding relations (36), (37) and (38) we simply notice that these hold when $x=x_{0}$ according to hypotheses (D1) and (D2), and the fact that the maps

$$
V_{R} \backslash B \rightarrow \mathbf{G}(3,2): x \mapsto W(\pi(x))=\operatorname{ker} D \pi(x)
$$

and

$$
V_{R} \rightarrow \mathbf{G}(3,2): x \mapsto T_{x} M_{d(x)}=\operatorname{ker} \nabla d(x)
$$

are continuous, so that these relations hold in a neighborhood of $x_{0}$ as well. With regard to inequality (39) we observe that $\theta(x)=\arccos \left|P_{W(\pi(x))}(u(x))\right|$ is continuous and that $\theta\left(x_{0}\right)>0$ for otherwise $u\left(x_{0}\right) \in T_{x_{0}} M_{r}$ in contradiction with hypothesis (D2). This clearly shows that $\theta(x) \geqslant \theta_{0}>0$ for $x$ in some neighborhood of $x_{0}$. Next we can assume that the choice of $e(x)$ is continuous in $x$. We let $v_{0}$ span $T_{\pi\left(x_{0}\right)} B=\left(W\left(\pi\left(x_{0}\right)\right)\right)^{\perp}$ and be such that

$$
\left\langle v_{0}, D \pi\left(x_{0}\right)\left(e\left(x_{0}\right)\right)\right\rangle>0
$$

(notice that necessarily $\left\langle v_{0}, D \pi\left(x_{0}\right)\left(e\left(x_{0}\right)\right)\right\rangle \neq 0$ for otherwise $e\left(x_{0}\right) \in \operatorname{ker} D \pi\left(x_{0}\right)=$ $W\left(\pi\left(x_{0}\right)\right)$ in contradiction with hypothesis (D2)). Since inequalities (40) and (41) depend continuously on $x$ we can restrict to a smaller neighborhood of $x_{0}$ where these hold as well. Q

Now we let $x, y \in\left(x_{0}+W_{0}\right) \cap \mathbf{U}\left(x_{0}, \rho_{1}\right) \backslash V_{r}$. In order to estimate the norm of $g_{r}(x)-g_{r}(y)$ we will estimate separately the norms of $g_{r}(x)-g_{r}(z)$ and $g_{r}(z)-g_{r}(y)$ for some point $z$ such that

$$
z \in M_{d(y)} \cap(\pi(x)+W(\pi(x))) \cap\left(x_{0}+W_{0}\right) .
$$

Before we start we need to estimate how far $z$ will be from $x$ and $y$ and to ensure the existence of a curve joining $z$ and $y$ on the "cylinder" $M_{d(y)}$.

Abbreviate

$$
\varepsilon=\frac{\sin \theta_{0}}{2}
$$

Claim \#2. There exist $C_{0}>0$ and $0<\rho_{2}<\rho_{1}$ with the following property. For every $0<\rho<\rho_{2}$ and every $x, y \in\left(x_{0}+W_{0}\right) \cap \mathbf{U}\left(x_{0}, \varepsilon \rho\right)$ such that $d(y) \geqslant \max \{d(x), r\}$ the set

$$
M_{d(y)} \cap(\pi(x)+W(\pi(x))) \cap\left(x_{0}+W_{0}\right) \cap \mathbf{U}\left(x_{0}, \rho\right)
$$

is a singleton, and if $z$ denotes its single element then

$$
\max \{|z-y|,|z-x|\} \leqslant C_{0}|x-y|
$$

$\mathbb{P}$ For each $x \in \mathbf{U}\left(x_{0}, \rho_{1}\right)$ the set $(\pi(x)+W(\pi(x))) \cap\left(x_{0}+W_{0}\right)$ is an affine line, say $L(x)$, according to (36). We notice that $x \in L(x)$. On the other hand the set $M_{d(y)} \cap(\pi(x)+$ $W(\pi(x))) \cap \mathbf{U}(\pi(x), R)$ is a circle centered at $\pi(x)$ of radius $d(y)$. We let $\left\{z_{1}, z_{2}\right\}=L(x) \cap$ $M_{d(y)} \cap \mathbf{U}(\pi(x), R)$. Since $z_{j}-x \in W(\pi(x)) \cap W_{0}$ we see that $z_{j}-x$ is a multiple of $u(x)$, $j=1,2$. We label $z_{1}, z_{2}$ so that $\left\langle z_{1}-x, n(x)\right\rangle \geqslant 0$. Projecting $z_{1}-x$ on $\operatorname{span}\{x-\pi(x)\}$ we infer from (39) that

$$
\left|z_{1}-x\right| \leqslant \frac{d(y)-d(x)}{\sin \theta(x)} \leqslant \frac{\varepsilon \rho}{\sin \theta_{0}} \leqslant \frac{\rho}{2}
$$

$4{ }^{\text {e }}$ SÉRIE - TOME $42-2009-\mathrm{N}^{\circ} 1$ 
whence $z_{1} \in \mathbf{U}\left(x_{0}, \rho\right)$ if $x, y \in \mathbf{U}\left(x_{0}, \varepsilon \rho\right)$ because $\left|x-x_{0}\right|<\varepsilon \rho \leqslant \rho / 2$. On the other hand, letting $x^{\prime}$ be such that $\left\{x, x^{\prime}\right\}=L(x) \cap M_{d(x)} \cap \mathbf{U}(\pi(x), R)$ we readily see that

$$
\left|z_{2}-x\right|^{2} \geqslant\left|x^{\prime}-x\right|^{2}=2 r^{2}(1-\cos 2 \theta(x))
$$

and, in turn,

$$
\left|z_{2}-x_{0}\right| \geqslant\left|z_{2}-x\right|-\left|x-x_{0}\right| \geqslant r \sqrt{2\left(1-\cos 2 \theta_{0}\right)}-\varepsilon \rho \geqslant \rho
$$

where the last inequality occurs provided $\rho<\rho_{2}$ and $\rho_{2}$ is chosen small enough depending upon $r, \theta_{0}$ and $\varepsilon$.

With regard to inequality (42) we recall from (43) that

$$
|z-x| \leqslant \frac{d(y)-d(x)}{\sin \theta(x)} \leqslant \frac{|y-x|}{\sin \theta_{0}}
$$

because $\operatorname{Lip} d \leqslant 1$. Also

$$
|z-y| \leqslant|z-x|+|x-y| \leqslant\left(\frac{1}{\sin \theta_{0}}+1\right)|x-y| .
$$

For use in the remaining part of this proof we let

$$
\Gamma_{\delta}=M_{\delta} \cap\left(x_{0}+W_{0}\right),
$$

$0<\delta<R$. We notice that $\Gamma_{\delta} \cap \mathbf{U}\left(x_{0}, \rho_{1}\right)$ is (either empty or) a 1-dimensional $C^{1}$ submanifold of $\mathbb{R}^{3}$ according to (37). Furthermore it follows from (38) that $\pi\left\lceil\Gamma_{\delta} \cap \mathbf{U}\left(x_{0}, \rho_{1}\right)\right.$ has everywhere a derivative of maximal rank. The following claim follows at once from these remarks.

ClaIm \#3. There exists $0<\rho_{3}<\varepsilon \rho_{2}$ such that $\pi \uparrow \Gamma_{r} \cap \mathbf{U}\left(x_{0}, \rho_{3}\right)$ is a $C^{1}$ diffeomorphism onto its image. We let $h$ denote its inverse.

Claim \#4. There exists $0<\rho_{4}<\rho_{3}$ with the following property. For every $0<\rho<\rho_{4}$ and every $x, y \in\left(x_{0}+W_{0}\right) \cap \mathbf{U}\left(x_{0}, \varepsilon \rho\right)$ with $d(y) \geqslant \max \{d(x), r\}$ if we let $z$ be such that

$$
\{z\}=M_{d(y)} \cap\left(x_{0}+W_{0}\right) \cap(\pi(x)+W(\pi(x))) \cap \mathbf{U}\left(x_{0}, \rho\right)
$$

(recall Claim \#2) then there exists a curve of finite length $\gamma:[a, b] \rightarrow \mathbb{R}^{3}$ such that $\gamma(a)=y$, $\gamma(b)=z$ and $\operatorname{im} \gamma \subseteq M_{d(y)} \cap\left(x_{0}+W_{0}\right) \cap \mathbf{U}\left(x_{0}, \rho_{1}\right)$.

$\mathbb{P}$ We choose $\rho_{4}$ small enough for $\pi\left(\mathbf{U}\left(x_{0}, \rho_{4}\right)\right) \subseteq \pi\left(\Gamma_{r} \cap \mathbf{U}\left(x_{0}, \rho_{3}\right)\right)$. Given $\rho, x$ and $y$ as in the statement we let $I$ denote the closed connected subset of $\pi\left(\mathbf{U}\left(x_{0}, \rho_{4}\right)\right)$ with endpoints $\pi(y)$ and $\pi(z)$. For each $\xi \in I$ we infer from Claim \#3 that there exists $x_{\xi} \in \Gamma_{r} \cap \mathbf{U}\left(x_{0}, \varepsilon \rho_{2}\right)$ such that $\pi\left(x_{\xi}\right)=\xi$. It follows in turn from Claim \#2 (applied with $x_{\xi}$ in place of $x$ ) that

$$
M_{d(y)} \cap(\xi+W(\xi)) \cap\left(x_{0}+W_{0}\right) \cap \mathbf{U}\left(x_{0}, \rho_{2}\right)
$$

is a singleton. In other words, letting

$$
E=M_{d(y)} \cap\left(x_{0}+W_{0}\right) \cap \mathbf{U}\left(x_{0}, \rho_{2}\right)
$$

we conclude that $\pi$ is a bijection from $E \cap \pi^{-1}(I)$ onto $I$. Since $E \cap \pi^{-1}(I) \subseteq \Gamma_{d(y)} \cap \mathbf{U}\left(x_{0}, \rho_{1}\right)$ is a compact subset, the inverse of the said map is Lipschitzian. The claim readily follows. $Q$

For the purpose of brevity we introduce the following convenient notation. For $\zeta \in V_{R} \backslash B$ we put

$$
q(\zeta)=\frac{\zeta-\pi(\zeta)}{d(\zeta)}
$$


We observe that for each $\zeta \in \mathbf{U}\left(x_{0}, r / 2\right) \backslash V_{r}$ we have $\lambda_{r}(d(\zeta))=d(\zeta)-r$ thanks to property (c) of $\lambda_{r}$, therefore

$$
g_{r}(\zeta)=\pi(\zeta)+(d(\zeta)-r)\left(\frac{\zeta-\pi(\zeta)}{d(\zeta)}\right)=\zeta-r q(\zeta)
$$

We set $\rho_{5}=\min \left\{\rho_{4}, r / 2\right\}$.

From now on we let $x, y \in\left(x_{0}+W_{0}\right) \cap \mathbf{U}\left(x_{0}, \varepsilon \rho_{j}\right) \backslash V_{r}$ (for $j=5,6, \ldots$ as we proceed in the proof) with $d(y) \geqslant d(x)$. We also let $z$ be associated with $x$ and $y$ as above.

Estimate of $\left|g_{r}(x)-g_{r}(z)\right|$. We write

$$
g_{r}(x)-g_{r}(z)=x-z-r(q(x)-q(z)) .
$$

Since each $(\xi+W(\xi)) \cap M_{r} \cap \mathbf{U}(\xi, R), \xi \in B$, is a circle of radius $r$ one checks that there exists $\rho_{6}>0$ such that for every $\zeta_{0} \in(\xi+W(\xi)) \cap M_{r} \cap \mathbf{U}(\xi, R)$ and every $\zeta_{1}, \zeta_{2} \in \mathbf{U}\left(\zeta_{0}, \rho_{6}\right) \cap$ $(\xi+W(\xi)) \backslash V_{r}$ one has

$$
r\left|\left\langle n\left(\zeta_{0}\right), q\left(\zeta_{1}\right)-q\left(\zeta_{2}\right)\right\rangle\right| \leqslant \varepsilon\left|\zeta_{1}-\zeta_{2}\right| .
$$

$\mathbb{P}$ By translating and rescaling we reduce to the case when $\xi=0, r=1, W(\xi)=$ $\operatorname{span}\left\{e_{1}, e_{2}\right\}, \zeta_{0}=e_{1}$, and $q(\zeta)=\zeta|\zeta|^{-1}$ for $\zeta \in W(\xi)$. Near $e_{1}$ the circle $W(\xi) \cap$ Bdry $\mathbf{B}(0,1)$ is a $C^{1}$ graph on its tangent line and therefore there exists $\rho_{6}>0$ such that

$$
\left|\left\langle e_{1}, q\left(\zeta_{1}\right)-q\left(\zeta_{2}\right)\right\rangle\right| \leqslant \varepsilon\left|\left\langle e_{2}, q\left(\zeta_{1}\right)-q\left(\zeta_{2}\right)\right\rangle\right|
$$

whenever $\zeta_{1}, \zeta_{2} \in W(\xi) \cap \mathbf{U}\left(e_{1}, \rho_{6}\right)$. If also $\zeta_{1}, \zeta_{2} \notin \mathbf{U}(0,1)$ then

$$
\left|\left\langle e_{1}, q\left(\zeta_{1}\right)-q\left(\zeta_{2}\right)\right\rangle\right| \leqslant \varepsilon\left|\left\langle e_{2}, q\left(\zeta_{1}\right)-q\left(\zeta_{2}\right)\right\rangle\right| \leqslant \varepsilon\left|q\left(\zeta_{1}\right)-q\left(\zeta_{2}\right)\right| \leqslant \varepsilon\left|\zeta_{1}-\zeta_{2}\right| .
$$

We define

$$
\zeta_{0}=\zeta_{0}(x)=\pi(x)+r\left(\frac{x-\pi(x)}{d(x)}\right)
$$

and we notice that $n\left(\zeta_{0}\right)=n(x)$. Recalling that $x-z \in W_{0} \cap W(\pi(x))$ (and is therefore a multiple of $u(x))$ and assuming that $x, z \in \mathbf{U}\left(\zeta_{0}, \rho_{6}\right)$ we obtain

$$
\begin{aligned}
\left|g_{r}(x)-g_{r}(z)\right| & \geqslant\left|\left\langle n\left(\zeta_{0}\right), g_{r}(x)-g_{r}(z)\right\rangle\right| \\
& \geqslant|\langle n(x), x-z\rangle|-r\left|\left\langle n\left(\zeta_{0}\right), q(x)-q(z)\right\rangle\right| \\
& \geqslant \sin \theta(x)|x-z|-\frac{\sin \theta_{0}}{2}|x-z| \geqslant \varepsilon|x-z| .
\end{aligned}
$$

We now claim that there exists $0<\rho_{7}<\rho_{5}$ such that if $x, y \in \mathbf{U}\left(x_{0}, \rho_{7}\right)$ then $x, z \in$ $\mathbf{U}\left(\zeta_{0}(x), \rho_{6}\right)$. This will imply that (47) holds provided $x, y \in \mathbf{U}\left(x_{0}, \varepsilon \rho_{7}\right)$. $\mathbf{P}$ Clearly

$$
\left|x-\zeta_{0}(x)\right|=\left(1-\frac{r}{d(x)}\right) \leqslant \frac{\rho}{R}
$$

and

$$
\left|z-\zeta_{0}(x)\right| \leqslant\left|x-\zeta_{0}(x)\right|+|x-z| \leqslant \frac{\rho}{R}+2 C_{0} \rho
$$

where we have referred to Claim \#2. The existence of $\rho_{7}$ follows at once. Q

Estimate of $\left|g_{r}(z)-g_{r}(y)\right|$. We let $\gamma:[a, b] \rightarrow \mathbb{R}^{3}$ be an arc length parametrization of the curve joining $y$ and $z$ in $M_{d(y)}$ as stated in Claim \#4. Notice that $\gamma^{\prime}(t)= \pm e(\gamma(t)), t \in[a, b]$, 
with the sign not changing as $t$ varies (so we may as well assume that $\gamma^{\prime}=e \circ \gamma$ by reversing the orientation of $\gamma$ if necessary). Writing $d(y)=r+\delta$ we observe that

$$
\begin{aligned}
g_{r}(z)-g_{r}(y) & =z-y-\frac{r}{r+\delta}(z-y-(\pi(z)-\pi(y))) \\
& =\frac{\delta}{r+\delta}(z-y)+\frac{r}{r+\delta} \int_{a}^{b} D \pi(\gamma(t))\left(\gamma^{\prime}(t)\right) d t \\
& =: v_{1}+v_{2} .
\end{aligned}
$$

Furthermore (40) yields

$$
\begin{aligned}
\left\langle v_{0}, v_{2}\right\rangle & =\frac{r}{r+\delta}\left\langle v_{0}, \int_{a}^{b} D \pi(\gamma(t))\left(\gamma^{\prime}(t)\right) d t\right\rangle=\frac{r}{r+\delta} \int_{a}^{b}\left\langle v_{0}, D \pi(\gamma(t))\left(\gamma^{\prime}(t)\right)\right\rangle d t \\
& \geqslant \frac{r \beta_{0}}{r+\delta} \operatorname{length}(\gamma) \geqslant \frac{r \beta_{0}}{r+\delta}|z-y| .
\end{aligned}
$$

Notice also that

$$
\left|\left\langle v_{0}, v_{1}\right\rangle\right| \leqslant\left|v_{1}\right|=\frac{r}{r+\delta}|z-y|
$$

Therefore

$$
\left\langle v_{0}, g_{r}(z)-g_{r}(y)\right\rangle \geqslant \frac{r \beta_{0}-\delta}{r+\delta}|z-y| .
$$

We infer that if $\delta \leqslant \min \left\{r, \beta_{0} r / 2\right\}$ then

$$
\left|g_{r}(z)-g_{r}(y)\right| \geqslant\left\langle v_{0}, g_{r}(z)-g_{r}(y)\right\rangle \geqslant \frac{\beta_{0}}{4}|z-y| .
$$

This can be achieved by requiring that $y \in \mathbf{U}\left(x_{0}, \rho_{8}\right)$ with

$$
\rho_{8}=\min \left\{\rho_{7}, r, \beta_{0} r / 2\right\} .
$$

Estimate of the angle between $g_{r}(x)-g_{r}(z)$ and $g_{r}(z)-g_{r}(y)$. We observe that $\pi(x)=\pi(z)$ and therefore $q(x), q(z) \in W(\pi(x))$. In turn $g_{r}(x)-g_{r}(z) \in W(\pi(x))$. Therefore

$$
\left.\left.\left\langle g_{r}(x)-g_{r}(z)\right\rangle, g_{r}(z)-g_{r}(y)\right\rangle=\left\langle g_{r}(x)-g_{r}(z)\right\rangle, P_{W(\pi(x))}\left(g_{r}(z)-g_{r}(y)\right)\right\rangle \text {. }
$$

Recalling (48) and (41), and abbreviating $u:=g_{r}(z)-g_{r}(y)$ we infer that

$$
\begin{aligned}
\left|P_{W(\pi(x))}\left(g_{r}(z)-g_{r}(y)\right)\right| & =\left|P_{W(\pi(x))}\left(\left\langle v_{0}, u\right\rangle v_{0}+\left(u-\left\langle v_{0}, u\right\rangle v_{0}\right)\right)\right| \\
& \leqslant|u|\left|P_{W(\pi(x))}\left(v_{0}\right)\right|+\sqrt{|u|^{2}-\left\langle v_{0}, u\right\rangle^{2}} \\
& \leqslant\left|g_{r}(z)-g_{r}(y)\right|\left(\left|P_{W(\pi(x))}\left(v_{0}\right)\right|+\sqrt{1-\frac{\beta_{0}^{2}}{16 \Lambda^{2}}}\right) \\
& =(1-\lambda)\left|g_{r}(z)-g_{r}(y)\right|
\end{aligned}
$$

where $\lambda:=\beta_{0}^{2} /\left(64 \Lambda^{2}\right)$. Consequently,

$$
\left|\left\langle g_{r}(x)-g_{r}(z)\right\rangle, g_{r}(z)-g_{r}(y)\right\rangle|\leqslant(1-\lambda)| g_{r}(x)-g_{r}(z)|| g_{r}(z)-g_{r}(y) \mid .
$$


Final estimate. We let $\rho_{0}=\varepsilon \rho_{8}$ and we observe that (47), (48) and (50) hold whenever $x, y \in\left(x_{0}+W_{0}\right) \mathbf{U}\left(x_{0}, \rho_{0}\right) \backslash V_{r}$. Whence

$$
\begin{aligned}
\left|g_{r}(x)-g_{r}(y)\right|^{2}=\left|g_{r}(x)-g_{r}(z)\right|^{2}+\left|g_{r}(z)-g_{r}(y)\right|^{2} & +2\left\langle g_{r}(x)-g_{r}(z), g_{r}(z)-g_{r}(y)\right\rangle \\
\geqslant & \begin{array}{c}
\left|g_{r}(x)-g_{r}(z)\right|^{2}+\left|g_{r}(z)-g_{r}(y)\right|^{2} \\
\quad-2(1-\lambda)\left|g_{r}(x)-g_{r}(z)\right|\left|g_{r}(z)-g_{r}(y)\right| \\
\geqslant
\end{array} \\
\geqslant & \frac{\lambda}{2}\left(\left|g_{r}(x)-g_{r}(z)\right|+\left|g_{r}(z)-g_{r}(y)\right|\right)^{2} \\
\geqslant & \frac{\beta_{0}^{2}}{128 \Lambda^{2}}\left(\varepsilon|x-z|+\frac{\beta_{0}}{4}|z-y|\right)^{2} .
\end{aligned}
$$

In turn

$$
\left|g_{r}(x)-g_{r}(y)\right| \geqslant \eta|x-y|
$$

where

$$
\eta=\frac{\beta_{0}}{12 \Lambda} \min \left\{\varepsilon, \frac{\beta_{0}}{4}\right\}
$$

and the proof of the theorem is complete.

12.4. Remark. - We now discuss the necessity of hypotheses (D1) and (D2). For that purpose we let $B=\operatorname{span}\left\{e_{1}\right\}$ (near the origin), $r=1$ and $x_{0}=e_{2}$.

(1) If $W_{0}=\operatorname{span}\left\{e_{1}, e_{3}\right\}=T_{x_{0}} M_{1}$ then the restriction of $g_{1}$ to any neighborhood of $x_{0}$ in $\left(x_{0}+W_{0}\right) \backslash V_{1}$ is clearly a Lipschitzian homeomorphism. However its inverse is no better than Hölder continuous of exponent $1 / 2$ (it suffices to notice that $g_{1}\left(e_{2}+t e_{3}\right)=$ $\left(1-\sqrt{1+t^{2}}\right) / \sqrt{1+t^{2}}$ when $t$ is close enough to 0$)$. It turns out that this situation is exceptional and we can avoid it by a "general position argument" to be made precise in the next section.

(2) The case when $W_{0}=\operatorname{span}\left\{e_{2}, e_{3}\right\}$, i.e. $W_{0}=W\left(\pi\left(x_{0}\right)\right)$, can also be ruled out generically, see next section.

(3) If $W_{0}=\operatorname{span}\left\{e_{3}, e_{1}+e_{2}\right\}$ then it is easily seen that $g_{1}$ is not a bijection from any neighborhood of $x_{0}$ in $\left(x_{0}+W_{0}\right) \backslash V_{1}$ onto its image - it is in fact two-to-one on $\left(x_{0}+W_{0}\right) \cap \operatorname{Bdry} V_{1}$ in a neighborhood of $x_{0}$. Most importantly, the restriction of $g_{1}$ to any neighborhood of $x_{0}$ in $\left\{x:\left\langle x, e_{3}\right\rangle \geqslant 0\right\} \cap\left(x_{0}+W_{0}\right) \backslash V_{1}$ is a bijection but its inverse is no better than Hölder continuous with exponent $1 / 2$ (same calculation as in (1) above). Unlike the two preceding cases, the present situation cannot be avoided by a general position argument (small translations or rotations of $W_{0}$ make the problem arise nearby on the curve $\left.\left(x_{0}+W_{0}\right) \cap \operatorname{Bdry} V_{1}\right)$. Instead we will "pleat" $W_{0}$.

\section{General position}

We now explain how to avoid the situation described in Remark 12.4(1).

13.1. Lemma. - Let $M \subseteq \mathbb{R}^{3}$ and let $E \subseteq \mathbb{R}^{3}$ be a finite or countable set. If there exists $r>0$ such that $(h+E) \cap M \neq \varnothing$ for every $h \in \mathbf{B}(0, r)$ then $\mathscr{L}^{3}(M)>0$.

$4^{\text {e }}$ SÉRIE - TOME $42-2009-\mathrm{N}^{\mathrm{o}} 1$ 
Proof. - For each $z \in E$ write $S_{z}=\mathbf{B}(0, r) \cap\{h: z+h \in M\}$. Then $\mathbf{B}(0, r)=$ $\cup_{z \in E} S_{z}$ and accordingly $\mathscr{L}^{3}\left(S_{z_{0}}\right)>0$ for some $z_{0} \in E$. Since $z_{0}+S_{z_{0}} \subseteq M$ the proof is complete.

13.2. Proposition. - Assume that $k=1,2, W \in \mathbf{G}(3, k)$ and $M \subseteq \mathbb{R}^{3}$ is a 2 dimensional compact submanifold of class $C^{k}$. Then the set

$$
G=\mathbb{R}^{3} \cap\left\{a: \operatorname{dim} W \cap T_{x} M=k-1 \text { for every } x \in(a+W) \cap M\right\}
$$

is open and dense.

Proof. - First notice that for every $V \in \mathbf{G}(3,2)$ either $\operatorname{dim} V \cap W=k-1$ or $\operatorname{dim} V \cap W=k$, the latter occurring if and only if $W \subseteq V$. Letting $B=\mathbb{R}^{3} \backslash G$ we proceed to showing that $B$ is closed. Assume $a_{1}, a_{2}, \cdots \in B$ and $a \in \mathbb{R}^{3}$ are such that $a_{j} \rightarrow a$ as $j \rightarrow \infty$. For each $j=1,2, \ldots$ there exists $x_{j} \in\left(a_{j}+W\right) \cap M$ such that $W \subseteq T_{x_{j}} M$. Since $M$ is compact there are $x \in M$ and a subsequence $k(1), k(2), \ldots$ of $1,2, \ldots$ such that $x_{j(k)} \rightarrow x$ as $k \rightarrow \infty$. Clearly $x \in(a+W) \cap M$. On the other hand $T_{x_{j(k)}} M \rightarrow T_{x} M$ (for instance locally in Hausdorff distance) because $M$ is of class $C^{1}$. Therefore $W \subseteq T_{x} M$.

It remains to prove that $G$ is dense. Let $a \in \mathbb{R}^{3}$. We consider the maps

$$
\tau_{-a}: M \rightarrow \mathbb{R}^{3}: x \mapsto x-a
$$

and

$$
\pi_{W}: \mathbb{R}^{3} \rightarrow \mathbb{R}^{3} / W
$$

(the natural projection). Both are obviously of class $C^{k}$. According to the Morse-Sard Theorem there are regular values $\mathbf{h}_{j} \in \mathbb{R}^{3} / W, j=1,2, \ldots$, of $\pi_{W} \circ \tau_{-a}$ such that $\mathbf{h}_{j} \rightarrow 0$ as $j \rightarrow \infty$. Choose $h_{j} \in \mathbb{R}^{3}$ such that $\pi_{W}\left(h_{j}\right)=\mathbf{h}_{j}, j=1,2, \ldots$, and $h_{j} \rightarrow 0$ as $j \rightarrow \infty$. We define $a_{j}=a+h_{j}$ and we will show that $a_{j} \in G, j=1,2, \ldots$ Indeed if $x \in\left(a_{j}+W\right) \cap M$ one easily checks that $\left(\pi_{W} \circ \tau_{-a_{j}}\right)(x)=\mathbf{h}_{j}$. Therefore the following linear map is surjective: $d\left(\pi_{W} \circ \tau_{-a_{j}}\right)(x)=\pi_{W} \circ d\left(\tau_{-a_{j}}\right)(x)=\pi_{W} \circ i_{T_{x} M}$ (where $i_{T_{x} M}: T_{x} M \rightarrow \mathbb{R}^{3}$ is the canonical injection). From this we infer that $T_{x} M+W=\mathbb{R}^{3}$, i.e. $\operatorname{dim} W \cap T_{x} M=k-1$.

\subsection{Theorem. - Assume that}

(A) $M \subseteq \mathbb{R}^{3}$ is a compact 2-dimensional submanifold of class 2;

(B) $P$ is a 2-dimensional nondegenerate polyhedral set in $\mathbb{R}^{3}$.

Then there exists an open dense set $D \subseteq \mathbb{R}^{3}$ such that for every $a \in D$ the following conditions hold.

(1) $\operatorname{dist}\left(M,(a+P) \cap\left\{x: \operatorname{card} \mathscr{W}_{a+P}(x) \geqslant 3\right\}\right)>0$, in particular for every $x \in(a+P) \cap M$ : card $\mathscr{W}_{a+P}(x) \leqslant 2$;

(2) For every $x \in(a+P) \cap M$ and every $W \in \mathscr{W}_{a+P}(x)$ : $\operatorname{dim} W \cap T_{x} M=1$;

(3) For every $x \in(a+P) \cap M$ such that card $\mathscr{W}_{a+P}(x)=2$ : $\operatorname{dim} W_{1} \cap W_{2} \cap T_{x} M=0$, where $\left\{W_{1}, W_{2}\right\}=\mathscr{W}_{a+P}(x)$. 
Proof. - For $j=1,2,3$ we let $D_{j}$ denote the set of $a \in \mathbb{R}^{3}$ such that conclusion (j) holds. It suffices to show that $D_{1}, D_{2}$ and $D_{3}$ each contain a subset which is open and dense in $\mathbb{R}^{3}$. We now introduce some notations for use in the present proof. For a given polyhedral set $Q$ we let $\mathbf{A}_{2, Q}$ denote the (finite) collection of 2-dimensional affine subspaces $Y$ of $\mathbb{R}^{3}$ such that $\mathscr{H}^{2}(Y \cap Q)>0$. We also let $\mathbf{A}_{1, Q}$ denote the (finite) collection of 1-dimensional affine subspaces $Z$ of $\mathbb{R}^{3}$ such that $Z=Y_{1} \cap Y_{2}$ for some $Y_{1}, Y_{2} \in \mathbf{A}_{2, Q}$. Finally we define $E_{Q}=$ $Q \cap\left\{x: \operatorname{card} \mathscr{W}_{Q}(x) \geqslant 3\right\}$.

From the nondegeneracy of $P$ we infer that each $x \in E_{P}$ is associated with some $Z_{1}, Z_{2} \in$ $\mathbf{A}_{1, P}$ such that $\{x\}=Z_{1} \cap Z_{2}$. Therefore $E_{P}$ is a finite set. Noticing that $E_{a+P}=a+E_{P}$ for every $a \in \mathbb{R}^{3}$, the openness of $D_{1}$ follows from the finiteness of $E_{P}$ and the closedness of $M$. That $D_{1}$ be dense is a consequence of Lemma 13.1.

For each $Y \in \mathbf{A}_{2, P}$ we select $h_{Y} \in Y$ and we apply Proposition 13.2 to $k=2, W=Y-h_{Y}$ and $M$. Denoting by $G_{Y}$ the set appearing in the conclusion of that proposition we observe that $D_{2}$ contains $\cap\left\{G_{Y}-h_{Y}: Y \in \mathbf{A}_{2, P}\right\}$ which is itself open and dense.

Similarly, for each $Z \in \mathbf{A}_{3, P}$ we select $h_{Z} \in Z$ and we apply Proposition 13.2 to $k=1$, $W=Z-h_{Z}$ and $M$. Letting $G_{Z}$ denote the set appearing in the conclusion of that proposition we note that $D_{3}$ contains $\cap\left\{G_{Z}-h_{Z}: Z \in \mathbf{A}_{1, P}\right\}$ which is open and dense.

We now show how to avoid the situation described in Remark 12.4(2).

\subsection{Lemma. - Assume that}

(A) $B \subseteq \mathbb{R}^{3}$ is a 1-dimensional compact submanifold of class $C^{1,1}$;

(B) $P \subseteq \mathbb{R}^{3}$ is a 2-dimensional polyhedral set.

Then there exists an open dense set $\mathscr{A} \subseteq \mathbf{O}(3)$ such that for every $A \in \mathscr{A}$ one has

$$
\mathscr{W}_{A(P)} \cap\{W(\pi(x)): x \in B\}=\varnothing .
$$

13.5. Remark. - Since $\mathscr{W}_{a+A(P)}=\mathscr{W}_{A(P)}$ whenever $a \in \mathbb{R}^{3}$ we see that in fact $\mathscr{W}_{a+A(P)} \cap\{W(\pi(x)): x \in B\}=\varnothing$ whenever $A \in \mathscr{A}$ and $a \in \mathbb{R}^{3}$.

Proof. - We consider the map $n: B \rightarrow \mathbf{G}(3,1): x \mapsto\left(T_{x} B\right)^{\perp}$ which is Lipschitzian according to assumption (A). Thus the Hausdorff dimension of im $n$ is 1 . Identifying $\mathbf{G}(3,1)$ to a quotient of $\mathbf{S}^{2}$ through the (bi-Lipschitzian) canonical map $\iota: \mathbf{S}^{2} \rightarrow \mathbf{G}(3,1)$ we recall that $\phi=\iota_{\#} \mathscr{H}^{2}$ is an $\mathbf{O}(3)$ invariant measure on $\mathbf{G}(3,1)$. It follows now that $\phi(\operatorname{im} n)=0$.

Letting $E=\mathbf{G}(3,1) \cap\left\{V: \mathscr{H}^{2}\left(\left(h+V^{\perp}\right) \cap P\right)>0\right.$ for some $\left.h \in \mathbb{R}^{3}\right\}$, and noticing that $E$ is finite, an argument similar to that of the proof of Lemma 13.1 implies that for every neighborhood $U$ of the identity in $\mathbf{O}(3)$ there exists $A \in U$ such that $(A \cdot E) \cap \operatorname{im} n=\varnothing$. The conclusion follows at once.

Next we establish the following. After applying the preceding lemma, the set of points where the worst situation occurs - with regard to hypothesis (D2) of Theorem 12.3 - is discrete. This is for use in the proof of Theorem 14.3.

13.6. Lemma. - Assume that

(A) $B \subseteq \mathbb{R}^{3}$ is a 1-dimensional compact submanifold of class $C^{3}$;

(B) $R$ and $M_{r}$ are as in section $12,0<r<R$;

$4^{\text {e }}$ SÉRIE - TOME $42-2009-\mathrm{N}^{\mathrm{o}} 1$ 
(C) $x_{0} \in M_{r}, W_{0} \in \mathbf{G}(3,2)$, $\operatorname{dim} W_{0} \cap T_{x_{0}} M_{r}=1$, $\operatorname{dim} W_{0} \cap T_{x_{0}} M_{r} \cap W\left(\pi\left(x_{0}\right)\right)=1$ and $W_{0} \neq W\left(\pi\left(x_{0}\right)\right)$.

Then there exists $\delta>0$ such that for every $x \in\left(x_{0}+W_{0}\right) \cap M_{r} \cap \mathbf{U}\left(x_{0}, \delta\right) \backslash\left\{x_{0}\right\}$ one has

$$
\operatorname{dim} W_{0} \cap T_{x} M_{r} \cap W(\pi(x))=0 .
$$

Proof. - Since $\operatorname{dim} W_{0} \cap T_{x_{0}} M_{r}=1$ we infer that the set $\Gamma=\left(x_{0}+W_{0}\right) \cap M_{r} \cap \mathbf{U}\left(x_{0}, \delta\right)$ is a 1-dimensional submanifold of class $C^{2}$, for some $\delta>0$. Let $I \subseteq \mathbb{R}$ be an open interval containing 0 and $\gamma: I \rightarrow \Gamma$ an arclength parametrization of $\Gamma$ such that $\gamma(0)=x_{0}$. The map $\pi \circ \gamma$ is of class $C^{2}$ according to Theorem 12.1(B) and our hypothesis (A). Since $(\pi \circ \gamma)^{\prime}(t)=$ $D \pi(\gamma(t))\left(\gamma^{\prime}(t)\right), t \in I$, we notice that $(\pi \circ \gamma)^{\prime}(t)=0$ if and only if $\gamma^{\prime}(t) \in \operatorname{ker} D \pi(\gamma(t))$, that is if and only $\gamma^{\prime}(t) \in W(\pi(\gamma(t)))$ (in view of Remark 12.2(4)), which in turn is equivalent to $\operatorname{dim} W_{0} \cap T_{\gamma(t)} M_{r} \cap W(\pi(\gamma(t)))=1$.

Differentiating twice we obtain

$$
(\pi \circ \gamma)^{\prime \prime}(0)=D^{2} \pi(\gamma(0))\left(\gamma^{\prime}(0), \gamma^{\prime}(0)\right)+D \pi(\gamma(0))\left(\gamma^{\prime \prime}(0)\right) .
$$

Since $\gamma^{\prime}(0) \in W_{0} \cap T_{x_{0}} M_{r} \subseteq W\left(\pi\left(x_{0}\right)\right)$ we deduce from Remark 12.2(4) and the very definition of $D^{2} \pi$ that $D^{2} \pi(\gamma(0))\left(\gamma^{\prime}(0), \gamma^{\prime}(0)\right)=0$. Next we claim that the second term above does not vanish. Since $\Gamma$ is a plane curve in $x_{0}+W_{0}$ and $\gamma$ an arclength parametrization we see that $\gamma^{\prime \prime}(0) \in W_{0} \cap\left(T_{x_{0}} M_{r} \cap W\left(\pi\left(x_{0}\right)\right)\right)^{\perp}$. Since $W_{0} \neq W\left(\pi\left(x_{0}\right)\right)$ according to our hypothesis (C), either $\gamma^{\prime \prime}(0)=0$ or $\gamma^{\prime \prime}(0) \notin W\left(\pi\left(x_{0}\right)\right)$. We next observe that $\gamma^{\prime \prime}(0) \neq 0$. This can be seen by differentiating twice the relation $r^{2}=d^{2}(\gamma(t)), t \in I$, and referring to Remark 12.2(3)

$$
\begin{aligned}
0 & =-\frac{1}{2} \frac{d^{2}}{d t^{2}} d^{2}(\gamma(t))_{t=0} \\
& =\left\langle D \pi(\gamma(0))\left(\gamma^{\prime}(0)\right)-\gamma^{\prime}(0), \gamma^{\prime}(0)\right\rangle+\left\langle D \pi(\gamma(0))-\gamma(0), \gamma^{\prime \prime}(0)\right\rangle \\
& =-1+\left\langle D \pi(\gamma(0))-\gamma(0), \gamma^{\prime \prime}(0)\right\rangle .
\end{aligned}
$$

Therefore $\gamma^{\prime \prime}(0) \notin \operatorname{ker} D \pi(\gamma(0))$ and we obtain $(\pi \circ \gamma)^{\prime \prime}(0) \neq 0$. The conclusion of the lemma then follows from the fundamental theorem of calculus applied to $(\pi \circ \gamma)^{\prime}$.

\section{Pleating}

We first describe the building block of the pleating. The deformation $h$ transforms $\left(x+W_{2}\right) \cap \mathbf{B}(x, \rho)$ into a set (a "pleat") which is polyhedral in a narrow slab around the plane $T$, close to the original $\left(x+W_{2}\right) \cap \mathbf{B}(x, \rho)$ (in area and Hausdorff distance), and uses only directions which are far from $W_{2}$. Moreover the deformation is the identity outside the ball $\mathbf{B}(x, \rho)$ and leaves the disk $\left(x+W_{1}\right) \cap \mathbf{B}(x, \rho)$ unchanged.

14.1. Lemma. - Assume that

(A) $W_{1}, W_{2}, T \in \mathbf{G}(3,2)$ and $\operatorname{dim} W_{1} \cap W_{2} \cap T=0$.

Then there exists $c\left(W_{1}, W_{2}, T\right)>0$ with the following property. Whenever

(B) $x \in \mathbb{R}^{3}$ and $U \subseteq \mathbb{R}^{3}$ is an open neighborhood of $x$;

(C) $0<\eta<1 / 6, \rho>0$ and $\mathbf{B}(x, \rho) \subseteq U$;

there exist a map $h: U \rightarrow U$ and a set $S^{*} \subseteq \mathbf{B}(x, \rho)$ such that the following hold. 
(D) $\operatorname{Lip} h \leqslant 2$, $h$ is $\rho$-homotopic to the identity of $U$ in the Lipschitzian category and is a bi-Lipschitzian homeomorphism of $U$ onto itself;

(E) There is a neighborhood $V$ of $x$ such that $\operatorname{Clos} V \subseteq \mathbf{U}(x, \rho)$ and $h(y)=y$ if $y \notin V$ whereas $h(y) \in V$ if $y \in V$;

(F) Letting $S=\left(x+W_{1}\right) \cup\left(x+W_{2}\right)$ one has

(F1) $h(S \cap V) \subseteq S^{*} \cap V$;

(F2) $\mathscr{H}^{2}\left(S^{*} \cap \mathbf{B}(x, \rho) \backslash h(S \cap \mathbf{B}(x, \rho))\right)<\eta$;

(F3) $S \cap(\mathbf{B}(x, \rho) \backslash V) \subseteq S^{*} \cap(\mathbf{B}(x, \rho) \backslash V)$;

(F4) $\operatorname{dist}_{\mathscr{H}}\left(S^{*} \cap \mathbf{B}(x, \rho), S \cap \mathbf{B}(x, \rho)\right)<2 \rho$;

(F5) $\mathscr{H}^{2}\left(S^{*} \cap V\right) \leqslant 2 \mathscr{H}^{2}(S \cap V)$;

(F6) $S^{*}$ is $\mathscr{H}^{2}$ essential;

(F7) There exists an open $\hat{V}$ with the following properties: Clos $V \subseteq \hat{V} \subseteq$ Clos $\hat{V} \subseteq$ $\mathbf{U}(x, \rho), S^{*} \cap \mathbf{B}(x, \rho) \backslash \hat{V}=S \cap \mathbf{B}(x, \rho) \backslash \hat{V}$ and $S^{*}$ is $(\overline{\mathbf{H}}, 1)$ locally connected at each point of $S^{*} \cap \operatorname{Clos} \hat{V}$;

(F8) $S^{*} \cap \mathbf{U}(x+T, \eta \rho) \cap \mathbf{U}(x, \rho)$ is 2-dimensional polyhedral;

(F9) If $y \in S^{*} \cap \mathbf{U}(x+T, \eta \rho) \cap$ Clos $\hat{V}$ the following hold: card $\mathscr{W}_{S^{*}}(y) \leqslant 2$ and for every $W \in \mathscr{W}_{S^{*}}(y)$ the following cases arise:

(a) $W=W_{1}$;

(b) $W_{1} \cap W_{2} \subseteq W$ (whence $\operatorname{dim} W \cap T=1$ ) and

$$
\operatorname{dist}\left(W \cap T, W_{2} \cap T\right)>\eta c\left(W_{1}, W_{2}, T\right) ;
$$

(c) $W=W_{2}$ and then $y \neq x$ and $y \in x+W_{2}$.

Proof. - We may assume that $x=0$. We let $u_{1}, u_{2}, u_{3}$ be a normed basis of $\mathbb{R}^{3}$ satisfying the requirement that $W_{1} \cap T=\operatorname{span}\left\{u_{1}\right\}, W_{2} \cap T=\operatorname{span}\left\{u_{2}\right\}$ and $W_{1} \cap W_{2}=\operatorname{span}\left\{u_{3}\right\}$. On letting $\mu=\max \left\{\left|\left\langle u_{1}, u_{2}\right\rangle\right|,\left|\left\langle u_{2}, u_{3}\right\rangle\right|,\left|\left\langle u_{1}, u_{3}\right\rangle\right|\right\}<1$ we see that

$$
\left|\sum_{i=1}^{3} t_{i} u_{i}\right|^{2}=\sum_{i, j=1}^{3} t_{i} t_{j}\left\langle u_{i}, u_{j}\right\rangle \geqslant(1-\mu) \sum_{i=1}^{3} t_{i}^{2},
$$

whenever $t_{1}, t_{2}, t_{3} \in \mathbb{R}$.

For $0<\delta<1$ we define an even function $\chi_{\delta, \rho}: \mathbb{R} \rightarrow \mathbb{R}$ by

$$
\chi_{\delta, \rho}(t)= \begin{cases}1 & \text { if } 0 \leqslant t \leqslant \delta \rho / 3 \\ \frac{3 t}{(1-\delta) \rho}-\frac{1}{1-\delta} & \text { if } \delta \rho / 3 \leqslant t \leqslant \rho / 3 \\ 0 & \text { if } \rho / 3 \leqslant t\end{cases}
$$

and we notice that $\left\|\chi_{\delta, \rho}\right\|_{\infty}=1$ and $\operatorname{Lip} \chi_{\delta, \rho}=\frac{3}{(1-\delta) \rho}$. Next we abbreviate

$$
\alpha=\frac{\eta \sqrt{1-\mu}}{9}
$$

and we define an odd function $p_{\alpha, \rho}: \mathbb{R} \rightarrow \mathbb{R}$ by

$$
p_{\alpha, \rho}(t)= \begin{cases}\alpha t & \text { if } 0 \leqslant t \leqslant \rho / 6 \\ -\alpha t+\frac{\alpha \rho}{3} & \text { if } \rho / 6 \leqslant t \leqslant \rho / 3 \\ 0 & \text { if } \rho / 3 \leqslant t\end{cases}
$$

$4^{\mathrm{e}}$ SÉRIE - TOME $42-2009-\mathrm{N}^{\mathrm{o}} 1$ 
and we notice that $\left\|p_{\alpha, \rho}\right\|_{\infty}=\frac{\alpha \rho}{6}$ and $\operatorname{Lip} p_{\alpha, \rho}=\alpha$. For convenience we set

$$
\varphi\left(t_{1}, t_{2}, t_{3}\right)=p_{\alpha, \rho}\left(t_{2}\right) \chi_{1 / 2, \rho}\left(t_{1}\right) \chi_{3 \eta, \rho}\left(t_{3}\right),
$$

$\left(t_{1}, t_{2}, t_{3}\right) \in \mathbb{R}^{3}$. Using the estimates stated above for $p_{\alpha, \rho}$ and $\chi_{\delta, \rho}$ we observe that $\operatorname{Lip} \varphi \leqslant 3 \alpha$. We are now ready to define $h: \mathbb{R}^{3} \rightarrow \mathbb{R}^{3}$ :

$$
h\left(\sum_{i=1}^{3} t_{i} u_{i}\right)=\left(t_{1}+\varphi\left(t_{1}, t_{2}, t_{3}\right)\right) u_{1}+t_{2} u_{2}+t_{3} u_{3} .
$$

We observe that

$$
\begin{aligned}
\left|h\left(\sum_{i=1}^{3} t_{i} u_{i}\right)-h\left(\sum_{i=1}^{3} t_{i}^{\prime} u_{i}\right)\right| & =\left|\sum_{i=1}^{3} t_{i} u_{i}-\sum_{i=1}^{3} t_{i}^{\prime} u_{i}-u_{1}\left(\varphi\left(t_{1}, t_{2}, t_{3}\right)-\varphi\left(t_{1}^{\prime}, t_{2}^{\prime}, t_{3}^{\prime}\right)\right)\right| \\
& \leqslant\left|\sum_{i=1}^{3} t_{i} u_{i}-\sum_{i=1}^{3} t_{i}^{\prime} u_{i}\right|+(\operatorname{Lip} \varphi) \sqrt{\sum_{i=1}^{3} t_{i}^{2}} \\
& \leqslant\left(1+\frac{3 \alpha}{\sqrt{1-\mu}}\right)\left|\sum_{i=1}^{3} t_{i} u_{i}-\sum_{i=1}^{3} t_{i}^{\prime} u_{i}\right| .
\end{aligned}
$$

Therefore

$$
\operatorname{Lip} h \leqslant 1+\frac{3 \alpha}{\sqrt{1-\mu}} \leqslant 2
$$

Furthermore we notice that

$$
\left|D_{1} \varphi\left(t_{1}, t_{2}, t_{3}\right)\right| \leqslant\left\|p_{\alpha, \rho}\right\|_{\infty}\left(\operatorname{Lip} \chi_{1 / 2, \rho}\right)\left\|\chi_{3 \eta, \rho}\right\|_{\infty}=\alpha .
$$

Thus for each fixed $t_{2}, t_{3} \in \mathbb{R}$ the Lipschitzian function

$$
\mathbb{R} \rightarrow \mathbb{R}: t_{1} \mapsto t_{1}+\varphi\left(t_{1}, t_{2}, t_{3}\right)
$$

has derivative (whenever it exists) bounded below by $1-\alpha$. A simple argument then shows that $h$ is bi-Lipschitzian. Moreover the open set

$$
V=\mathbb{R}^{3} \cap\left\{: \sum_{i=1}^{3} t_{i} u_{i}:\left|t_{i}\right|<\rho / 3, i=1, \ldots, 3\right\}
$$

is contained in $\mathbf{U}(0, \rho)$ and $h(y)=y$ whenever $y \notin V$. Trivially $\left|t_{1}+\varphi\left(t_{1}, t_{2}, t_{3}\right)\right|<\rho / 3$ when $\left|t_{1}\right|<\rho / 3$ so that $h(y) \in V$ when $y \in V$. Since $\mathbf{B}(0, \rho) \subseteq U$ this in turn implies that $h$ is a bi-Lipschitzian homeomorphism of $U$ onto itself. Now the map

$$
H:[0,1] \times U \rightarrow U:\left(\tau, \sum_{i=1}^{3} t_{i} u_{i}\right) \mapsto\left(t_{1}+\tau \varphi\left(t_{1}, t_{2}, t_{3}\right)\right) u_{1}+t_{2} u_{2}+t_{3} u_{3}
$$

witnesses the fact that $h$ is $\rho$-homotopic to the identity of $U$ in the Lipschitzian category (because $\|\varphi\|_{\infty} \leqslant \alpha \rho / 6 \leqslant \rho$ ). Thus the proof of conclusions (D) and (E) is complete.

We now turn to proving conclusion (F). We start by observing that $h\left(W_{1} \cap \operatorname{Clos} V\right)=$ $W_{1} \cap \operatorname{Clos} V$. P If $y \in \operatorname{Clos} V$ then $h(y) \in \operatorname{Clos} V$ as we saw above. Since $\sum_{i=1}^{3} t_{i} u_{i} \in W_{1}$ if and only if $t_{2}=0$ it is obvious that $y \in W_{1}$ implies $h(y) \in W_{1}$. Therefore 
$h\left(W_{1} \cap \operatorname{Clos} V\right) \subseteq W_{1} \cap \operatorname{Clos} V$. Since also $h \uparrow W_{1} \cap$ Bdry $V=\operatorname{id}_{W_{1} \cap \operatorname{Bdry} V}$ the conclusion follows from the fact that $h\left(W_{1} \cap\right.$ Clos $\left.V\right)$ is simply connected. Q Next we define

$$
\begin{aligned}
& u^{+}=\frac{u_{2}+\alpha u_{1}}{\left|u_{2}+\alpha u_{1}\right|} \\
& u^{-}=\frac{u_{2}-\alpha u_{1}}{\left|u_{2}-\alpha u_{1}\right|}
\end{aligned}
$$

and $W^{+}=\operatorname{span}\left\{u^{+}, u_{3}\right\}, W^{-}=\operatorname{span}\left\{u^{-}, u_{3}\right\}$. Notice that $W_{1} \cap W_{2} \subseteq W^{+}$and $W_{1} \cap W_{2} \subseteq$ $W^{-}$. Also, $W^{+} \cap T=\operatorname{span}\left\{u^{+}\right\}$and $W^{-} \cap T=\operatorname{span}\left\{u^{-}\right\}$. Therefore

$$
\begin{aligned}
\operatorname{dist}\left(W^{+} \cap T, W_{2} \cap T\right) & =\sqrt{1-\left\langle u^{+}, u_{2}\right\rangle^{2}}=\sqrt{1-\frac{\left(1+\alpha\left\langle u_{1}, u_{2}\right\rangle\right)^{2}}{1+2 \alpha\left\langle u_{1}, u_{2}\right\rangle+\alpha^{2}}} \\
& \geqslant \alpha \frac{\sqrt{1-\left\langle u_{1}, u_{2}\right\rangle^{2}}}{2}=\eta \frac{\sqrt{(1-\mu)\left(1-\left\langle u_{1}, u_{2}\right\rangle^{2}\right)}}{18} .
\end{aligned}
$$

This lower bound also holds with $W^{+}$replaced by $W^{-}$.

We notice that $h\left(W_{2}\right) \cap \mathbf{U}(T, \eta \rho)=h\left(W_{2} \cap \mathbf{U}(T, \eta \rho)\right)$. $\mathbb{P}$ Check that $\sum_{i=1}^{3} t_{i} u_{i} \in$ $\mathbf{U}(T, \eta \rho)$ if and only if $\left|t_{3}\right|<\eta \rho$, so that $h(y) \in \mathbf{U}(T, \eta \rho)$ if and only if $y \in \mathbf{U}(T, \eta \rho)$ for every $y \in \mathbb{R}^{3}$. Therefore $h\left(W_{2}\right) \cap \mathbf{U}(T, \eta \rho)=h\left(W_{2} \cap \mathbf{U}(T, \eta \rho)\right)$. Q We define $E=h\left(W_{2} \cap\right.$ $\mathbf{U}(T, \eta \rho))$. Next we observe that $h \nmid W_{2} \cap \mathbf{U}(T, \eta \rho)$ is piecewise affine because if $\sum_{i=1}^{3} t_{i} u_{i} \in$ $W_{2} \cap \mathbf{U}(T, \eta \rho)$ then

$$
h\left(\sum_{i=1}^{3} t_{i} u_{i}\right)=\left(t_{1}+p_{\alpha, \rho}\left(t_{1}\right)\right) u_{1}+t_{2} u_{2}+t_{3} u_{3} .
$$

Thus

$$
\begin{aligned}
E=\mathbf{U}(T, \eta \rho) \cap\left\{\left(W_{2} \cap \operatorname{Clos} V\right)\right. & \cup\left(W^{+} \cap\left\{\sum_{i=1}^{3} t_{i} u_{i}:\left|t_{2}\right| \leqslant \rho / 6\right\}\right) \\
& \cup\left(\left(a+W^{-}\right) \cap\left\{\sum_{i=1}^{3} t_{i} u_{i}: \rho / 6 \leqslant t_{2} \leqslant \rho / 3\right\}\right) \\
& \left.\cup\left(\left(b+W^{-}\right) \cap\left\{\sum_{i=1}^{3} t_{i} u_{i}:-\rho / 3 \leqslant t \leqslant-\rho / 6\right\}\right)\right\}
\end{aligned}
$$

for some appropriate $a, b \in \mathbb{R}^{3} \backslash\{0\}$. Note this set is not 2-dimensional polyhedral (because its intersection with $W^{+}$e.g. is not relatively open).

Therefore we call $E^{*} \subseteq \mathbf{U}(T, \eta \rho)$ some slight enlargement of $E$ verifying the following properties:

(1) $E \subseteq E^{*}$;

(2) $\mathscr{H}^{2}\left(E^{*}\right)<\eta+\mathscr{H}^{2}(E)$;

(3) $E \backslash \mathbf{U}(V, \eta \rho)=S \cap \mathbf{U}(T, \eta \rho) \backslash \mathbf{U}(V, \eta \rho)$;

(4) $E^{*}$ is $\mathscr{H}^{2}$ essential;

(5) $E^{*}$ is 2-dimensional polyhedral and card $\mathscr{W}_{E^{*}}(y) \leqslant 2$ for every $y \in \mathscr{W}_{E^{*}}(y)$;

(6) For every $y \in E^{*} \cap \mathbf{U}(V, \eta \rho), \mathscr{W}_{E^{*}}(y) \subseteq\left\{W^{+}, W^{-}\right\}$and $\mathscr{W}_{E^{*}}(y)=\left\{W^{+}\right\}$if $y \in$ $E \cap W_{1}$. 
( $E^{*}$ is obtained from $E$ by addition of narrow affine slabs near the four line segments constituting the 1-dimensional skeleton of $E$ ). We now put

$$
S^{*}=\left(W_{1} \cap \mathbf{B}(0, \rho)\right) \cup\left(E^{*} \cap \mathbf{B}(0, \rho)\right) \cup\left(h\left(W_{2} \cap \mathbf{B}(0, \rho)\right) \backslash \mathbf{U}(T, \eta \rho)\right) .
$$

Conclusion (F1) follows from the invariance of $W_{1}$ under $h$, and (1) above. Conclusion (F2) follows from (2) above. Conclusion (F3) follows from the definition of $S^{*}$, (1) above and the fact that $h$ coincides with the identity outside of $V$. Conclusion (F4) is most trivial. With regard to conclusion (F5) we observe that

$$
\mathscr{H}^{2}\left(h\left(W_{2}\right) \cap V\right)=\mathscr{H}^{2}\left(h\left(W_{2} \cap V\right)\right) \leqslant(\operatorname{Lip} h)^{2} \mathscr{H}^{2}\left(W_{2} \cap V\right)
$$

and that $(\operatorname{Lip} h)^{2} \leqslant 2$ in view of (51). Conclusion (F6) follows from (4) above. For conclusion (F7) to hold true we let $\hat{V}=\mathbf{U}(V, \eta \rho)$ and we refer to (3) above and to the fact that $h=\mathrm{id}_{\mathbb{R}^{3}}$ outside of $V$ for showing that $S^{*} \cap \mathbf{B}(0, \rho) \backslash \hat{V}=S \cap \mathbf{B}(0, \rho) \backslash \hat{V}$. Let $y \in S^{*} \cap$ Clos $\hat{V}$. If $\operatorname{dist}(y, T) \neq \eta \rho$ then $S^{*}$ coincides with $h(S)$ in a neighborhood of $y$ and is therefore $(\overline{\mathbf{H}}, 1)$ locally connected at $y$. If $\operatorname{dist}(y, T)=\eta \rho$ then the $(\overline{\mathbf{H}}, 1)$ local connectedness of $S^{*}$ at $y$ follows also on noticing that a neighborhood of $y$ in $S^{*}$ is contractible in the Lipschitzian category (this requires some straightforward checking that we leave to the reader). Finally, conclusions (F8) and (F9) are immediate consequences of (5) and (6) above together with inequality (52).

14.2. REMARK. - It is virtually obvious that the above proof also proves the (easier) statement when the set $S$ (in (F)) is replaced by $S=x+W_{2}$ (one then simply omits the piece $W_{1} \cap \mathbf{B}(0, \rho)$ in the definition of $\left.S^{*}\right)$. Under those circumstances the case (a) in (F9) does not arise.

\subsection{TheOrem. - Assume that}

(A) $B \subseteq \mathbb{R}^{3}$ is a 1-dimensional compact submanifold of class $C^{2}$ and $R>0, \pi$ and $W$ are associated with $B$ as in Section 12;

(B) $0<r<R / 4$ and $M_{r}=$ Bdry $V_{r}$ is as in Section 12;

(C) $P \subseteq \mathbb{R}^{3}$ is a 2-dimensional nondegenerate polyhedral set and is $\mathscr{H}^{2}$ essential;

(D) $U \subseteq \mathbb{R}^{3}$ is an open set containing Clos $P$;

(E) $\varepsilon>0$.

Then there exist a set $Q \subseteq U$ and a Lipschitzian map $h: U \rightarrow U$ with the following properties.

(F) $\operatorname{Lip} h \leqslant 2$, $h$ is a bi-Lipschitzian homeomorphism of a neighborhood of Clos $P$ onto its image, it is $\varepsilon$-homotopic to the identity of $U$ in the Lipschitzian category;

(G) $h(P) \subseteq Q$ and $\mathscr{H}^{2}(Q \backslash h(P))<\varepsilon$;

(H) $\operatorname{dist}_{\mathscr{H}}(P, Q)<\varepsilon$;

(I) There exist $a \in \mathbb{R}^{3}$ and $A \in \mathbf{O}(3)$ with $|a|<\varepsilon$, $\left\|A-\operatorname{id}_{\mathbb{R}^{3}}\right\|<\varepsilon$ and $\mathscr{H}^{2}(h(P) \triangle(a+$ $A(P)))<\varepsilon$

(J) $\left|\mathscr{H}^{2}(P)-\mathscr{H}^{2}(Q)\right|<\varepsilon$;

(K) $Q$ is $\mathscr{H}^{2}$ essential;

(L) $Q$ is $(\overline{\mathbf{H}}, 1)$ locally connected;

(M) There exists $\delta>0$ such that $Q \cap \mathbf{U}\left(M_{r}, \delta\right)$ is 2-dimensional polyhedral and for every $x \in M_{r} \cap Q$ the following hold: 
(M1) $\operatorname{card} \mathscr{W}_{Q}(x) \leqslant 2$; ${ }^{(17)}$

(M2) For every $W \in \mathscr{W}_{Q}(x)$ one has $\operatorname{dim} W \cap T_{x} M_{r}=1$;

(M3) For every $W \in \mathscr{W}_{Q}(x)$ one has $\operatorname{dim} W \cap T_{x} M_{r} \cap W(\pi(x))=0$;

(M4) If $\operatorname{card} \mathscr{W}_{Q}(x)=2$ and $\left\{W_{1}, W_{2}\right\}=\mathscr{W}_{Q}(x)$ then

$$
\operatorname{dim} W_{1} \cap W_{2} \cap T_{x} M_{r}=0 .
$$

Proof. - Let $\delta=\operatorname{dist}(P, \operatorname{Bdry} U)$. We first choose $A \in \mathscr{A} \cap \mathbf{S O}(3)$ such that $\left\|A-\operatorname{id}_{\mathbb{R}^{3}}\right\|<\min \{\varepsilon / 4, \delta / 4\}$ where $\mathscr{A}$ is associated with $B$ and $P$ in Lemma 13.4. For $x \in U$ we define $h_{A}(x)=\mathbf{A}(\operatorname{dist}(x, \operatorname{Bdry} U))(x)$ where $\mathbf{A}:(0, \infty) \rightarrow \mathbf{O}(3)$ is Lipschitzian and such that $\left\|\mathbf{A}(t)-\operatorname{id}_{\mathbb{R}^{3}}\right\|<t$ for every $t>0$ and $\mathbf{A}(t)=A$ whenever $t \geqslant \delta / 2$. One checks that $h_{A}(P)=A(P), h_{A}: U \rightarrow U$ is a bi-Lipschitzian homeomorphism of a neighborhood of $P$ onto its image and is $\varepsilon / 4$-homotopic to the identity of $U$ in the Lipschitzian category. Notice that $\operatorname{dist}(A(P), \operatorname{Bdry} U)>3 \delta / 4$. We choose $a \in D \cap \mathbf{U}(0, \min \{\varepsilon / 4, \delta / 4\})$ where $D$ is associated with $M_{r}$ and $A(P)$ in Theorem 13.3. For $x \in U$ we define $h_{a}(x)=x+a \chi(\operatorname{dist}(x, \operatorname{Bdry} U))$ where $\chi$ : $(0, \infty) \rightarrow(0, \infty)$ is a $C^{\infty}$ function such that $\chi(t)=1$ if $t \geqslant \delta / 4, \chi$ is strictly increasing, Lipschitzian and $\chi(t) \rightarrow 0$ as $t \rightarrow 0$. One easily checks that $h_{a}(A(P))=$ $a+A(P), h_{a}: U \rightarrow U$ is a bi-Lipschitzian homeomorphism of a neighborhood of $A(P)$ onto its image, and is $\varepsilon / 4$-homotopic to the identity of $U$ in the Lipschitzian category. We notice that $h_{a} \circ h_{A}: U \rightarrow U$ is a bi-Lipschitzian homeomorphism of a neighborhood of $P$ onto its image, and is $\varepsilon / 2$-homotopic to the identity of $U$ according to Remark 10.7 .

For a set $E \subseteq \mathbb{R}^{3}$ which is 2-dimensional polyhedral in a neighborhood of $M_{r}$ we let

$$
G(E)=M_{r} \cap E \cap\left\{x: \operatorname{card} \mathscr{W}_{E}(x) \leqslant 2 \text { and } \operatorname{dim} W \cap T_{x} M_{r} \cap W(\pi(x))=0\right.
$$

$$
\text { for every } \left.W \in \mathscr{W}_{E}(x)\right\}
$$

and

$$
\begin{aligned}
& B(E)=M_{r} \cap E \cap\left\{x: \operatorname{card} \mathscr{W}_{E}(x) \leqslant 2 \text { and } \operatorname{dim} W \cap T_{x} M_{r} \cap W(\pi(x))=1\right. \\
&\text { for some } \left.W \in \mathscr{W}_{E}(x)\right\} .
\end{aligned}
$$

Readily $G(E) \cap B(E)=\varnothing$ and $M_{r} \cap E \cap\left\{x: \operatorname{card} \mathscr{W}_{E}(x) \leqslant 2\right\}=G(E) \cup B(E)$. We further consider two disjoint parts of $B(E)$ :

$$
\begin{aligned}
& B_{1}(E)=B(E) \cap\left\{x: \operatorname{card} \mathscr{W}_{E}(x)=1\right\}, \\
& B_{2}(E)=B(E) \cap\left\{x: \operatorname{card} \mathscr{W}_{E}(x)=2\right\} .
\end{aligned}
$$

Notice that $B(a+A(P))=B_{1}(a+A(P)) \cup B_{2}(a+A(P))$ according to Theorem 13.3(1). We claim that

$$
\begin{aligned}
& B_{2}(a+A(P))=M_{r} \cap(a+A(P)) \cap\left\{x: \operatorname{card} \mathscr{W}_{a+A(P)}(x)=2\right. \text { and } \\
& \quad \text { there is a numbering }\left\{W_{1}(x), W_{2}(x)\right\} \text { of } \mathscr{W}_{a+A(P)}(x) \text { such that } \\
& \left.\quad \operatorname{dim} W_{1}(x) \cap T_{x} M_{r} \cap W(\pi(x))=0 \text { and } \operatorname{dim} W_{2}(x) \cap T_{x} M_{r} \cap W(\pi(x))=1\right\} .
\end{aligned}
$$

(From now on $W_{1}(x)$ and $W_{2}(x)$ will be given the meaning from the above definition whenever $\left.x \in B_{2}(a+A(P))\right)$. $\mathbb{P}$ Let $x \in M_{r} \cap(a+A(P))$ be so that card $\mathscr{W}_{a+A(P)}(x)=2$ and

${ }^{(17)}$ Here and subsequently we abuse notation by writing $\mathscr{W}_{Q}(x)$ instead of $\mathscr{W}_{Q \cap U\left(M_{r}, \delta\right)}(x)$.

$4^{\mathrm{e}}$ SÉRIE - TOME $42-2009-\mathrm{N}^{\mathrm{o}} 1$ 
write $\mathscr{W}_{a+A(P)}(x)=\left\{W_{1}, W_{2}\right\}$. We notice that $\operatorname{dim} W_{j} \cap T_{x} M_{r} \cap W(\pi(x)) \leqslant \operatorname{dim} T_{x} M_{r} \cap$ $W(\pi(x))=1, j=1,2$. Assume if possible that $\operatorname{dim} W_{j} \cap T_{x} M_{r} \cap W(\pi(x))=1$ for $j=1,2$. Since $\operatorname{dim} W_{1} \cap W_{2}=1$ we would have that $W_{1} \cap W_{2} \subseteq T_{x} M_{r}$ in contradiction with Theorem 13.3(3). @ Notice that if $B(a+A(P))=\varnothing$ then the proof of the theorem is complete (letting $h=h_{a} \circ h_{A}$ and $Q=h(P)$ ). We henceforth assume that this set is not empty and we will define a map $h_{b}$ to be a small perturbation of the identity and that "pleats" $a+A(P)$ near each point of $B(a+A(P))$, in such a way that $B\left(h_{b}(a+A(P))\right)=\varnothing$.

We intend to show that $B(a+A(P))$ is finite. For technical convenience we consider a 2-dimensional polyhedral set $\hat{P}$ in $\mathbb{R}^{3}$ with the following properties: Clos $P \subseteq \hat{P}$ and $\operatorname{card} \mathscr{W}_{a+A(\hat{P})}(x) \leqslant 2$ for every $x \in M_{r} \cap(a+A(\hat{P}))$, and conclusions (2) and (3) of Theorem 13.3 hold with $a+A(P)$ replaced by $a+A(\hat{P})$ (that this be possible follows from the fact that conclusion (1) of Theorem 13.3 holds for $a+A(P)$, and the continuity of the maps $M_{r} \rightarrow \mathbf{G}(3,2): y \mapsto T_{y} M_{r}$ and $\left.y \mapsto W(\pi(x))\right)$. Since readily $(a+A(\operatorname{Clos} P)) \cap B(a+A(\hat{P})) \supseteq B(a+A(P))$ it is sufficient to show that the former is finite.

Note that the set $B(a+A(\hat{P}))$ is discrete (and, as a consequence, so is $(a+A(\operatorname{Clos} P)) \cap$ $B(a+A(\hat{P})))$. $\mathbb{P}$ Let $x \in B(a+A(\hat{P}))$ and select $\rho>0$ so that

$$
\begin{aligned}
& (a+A(\hat{P})) \cap \mathbf{U}(x, \rho) \\
& \qquad= \begin{cases}(x+W) \cap \mathbf{u}(x, \rho) & \text { if } \mathscr{W}_{a+A(\hat{P})}(x)=\{W\} \\
\left(x+W_{1}(x)\right) \cup\left(x+W_{2}(x)\right) \cap \mathbf{U}(x, \rho) & \text { if } \operatorname{card}_{a+A(\hat{P})}(x)=2 .\end{cases}
\end{aligned}
$$

Assume if possible that there are $x_{j} \in B(a+A(\hat{P})) \cap \mathbf{U}(x, \rho), j=1,2, \ldots$, distinct from $x$ and such that $x_{j} \rightarrow x$ as $j \rightarrow \infty$. We need to consider several cases separately. First assume that $x_{j} \in B_{2}(a+A(P))$ for infinitely many $j$ (and hence for all $j$ without loss of generality). Then necessarily $x \in B_{2}(a+A(\hat{P}))$. In that case we observe that $x_{j}-x \in W_{1}(x) \cap W_{2}(x)$. In other words $x_{j}=x+t_{j} e$ (with $\left.t_{j} \neq 0\right), j=1,2, \ldots$, where $e$ is such that $\operatorname{span}\{e\}=$ $W_{1}(x) \cap W_{2}(x)$. On the other hand $d\left(x_{j}\right)=r=d(x)$ since $x_{j} \in B(a+A(\hat{P})) \subseteq M_{r}$ and from Taylor's formula

$$
d\left(x+t_{j} e\right)=d(x)+t_{j}\langle e, \nabla d(x)\rangle+o\left(t_{j}\right),
$$

letting $j \rightarrow \infty$, we infer that $\langle e, \nabla d(x)\rangle=0$. This means that $W_{1}(x) \cap W_{2}(x) \subseteq T_{x} M_{r}$. Now we choose $e^{\prime} \in W_{2}(x) \backslash\{0\}$ so that $e^{\prime} \in T_{x} M_{r} \cap W(\pi(x))$ and we notice that $e^{\prime} \notin W_{1}(x)$ (the choice is possible precisely because $x \in B(a+A(\hat{P}))$ ). We conclude that $e$ and $e^{\prime}$ are linearly independent, and in turn that $W_{2}(x) \subseteq T_{x} M_{r}$, in contradiction with Theorem 13.3(2). Therefore we conclude that $x_{j} \in B_{1}(a+A(\hat{P}))$ for infinitely many $j$. In that case we choose $W_{0} \in \mathscr{W}_{a+A(\hat{P})}(x)$ such that $x_{j} \in x+W_{0}$ for infinitely many $j$ (and hence for all $j$ without loss of generality). This is trivially possible in case card $\mathscr{W}_{a+A(\hat{P})}(x)=1$ and relies on the pigeonhole principle in case card $\mathscr{W}_{a+A(\hat{P})}(x)=2$. Since $\operatorname{dim} W_{0} \cap T_{x_{j}} M_{r} \cap W\left(\pi\left(x_{j}\right)\right)=1$ for every $j$, the continuity of the map $\mathbf{U}(B, R) \rightarrow \mathbf{G}(3,1): y \mapsto T_{y} M_{r} \cap W(\pi(y))$ would imply that $\operatorname{dim} W_{0} \cap T_{x} M_{r} \cap W(\pi(x))=1$. Since $W_{0} \neq W(\pi(x))$ according to Lemma 13.4, this would contradict Lemma 13.6. @

Next we note that the set $(a+A(\operatorname{Clos} P)) \cap B(a+A(\hat{P}))$ is closed. $\mathbb{P}$ Let $x_{j} \in$ $(a+A(\operatorname{Clos} P)) \cap B(a+A(\hat{P})), j=1,2, \ldots$, and $x \in M_{r}$ be such that $x_{j} \rightarrow x$ as $j \rightarrow \infty$. It 
is clear that $x \in a+A(\operatorname{Clos} P)$. Notice that $\mathscr{W}_{a+A(\hat{P})}\left(x_{j}\right) \subseteq \mathscr{W}_{a+A(\hat{P})}(x)$ if $j$ is large enough (a general fact about polyhedral sets). Arguing as before we choose $W_{0} \in \mathscr{W}_{a+A(\hat{P})}\left(x_{j}\right)$ independent of $j$ such that $\operatorname{dim} W_{0} \cap T_{x_{j}} M_{r} \cap W\left(\pi\left(x_{j}\right)\right)=1$ for every $j$. The continuity of the map $y \mapsto T_{y} M_{r} \cap W(\pi(y))$ thus ensures that $\operatorname{dim} W_{0} \cap T_{x} M_{r} \cap W(\pi(x))=1$, i.e. $x \in B(a+A(\hat{P}))$. Q

Now since $(a+A(\operatorname{Clos} P)) \cap B(a+A(\hat{P}))$ is a discrete closed subset of $M_{r}$ we infer that it is finite. This implies indeed that $B(a+A(P))$ is finite as well. Put $B(a+A(P))=\left\{x_{i}\right.$ : $\left.i=1, \ldots, x_{\kappa}\right\}$. In case $x_{i} \in B_{2}(a+A(P)), i=1, \ldots, \kappa$, we give $W_{1}\left(x_{i}\right)$ and $W_{2}\left(x_{i}\right)$ the same meaning as in the definition of $B_{2}(a+A(P))$, otherwise we adopt the convention that $\left\{W_{2}\left(x_{i}\right)\right\}=\mathscr{W}_{a+A(P)}(x)$ and $W_{1}\left(x_{i}\right)$ is any fixed plane such that $\operatorname{dim} W_{1}\left(x_{i}\right) \cap T_{x_{i}} M_{r}=1$, $\operatorname{dim} W_{1}\left(x_{i}\right) \cap T_{x_{i}} M_{r} \cap W\left(\pi\left(x_{i}\right)\right)=0$ and $\operatorname{dim} W_{1}\left(x_{i}\right) \cap W_{2}\left(X_{i}\right) \cap T_{x_{i}} M_{r}=0$.

We choose

$$
0<\rho_{0}<\min \left\{\frac{\varepsilon}{8}, \sqrt{\frac{\varepsilon}{12 \kappa \pi}}\right\}
$$

small enough for $\rho_{0}<\left|x_{i}-x_{j}\right| / 4$ whenever $i, j=1, \ldots, \kappa$ with $i \neq j$, and $\mathbf{B}\left(x_{i}, \rho_{0}\right) \subseteq U$, $i=1, \ldots, \kappa$. We let

$$
0<\eta<\min \left\{\frac{\varepsilon}{1+\mathscr{H}^{2}(P)}, \frac{\varepsilon}{2 \kappa}\right\} .
$$

For each $i=1, \ldots, \kappa$ we also define

$$
\beta_{i}=\left|P_{\left(T_{x_{i}} M r\right)^{\perp}}\left(u_{i}\right)\right|
$$

where $u_{i} \in W_{1}\left(x_{i}\right) \cap W_{2}\left(x_{i}\right),\left|u_{i}\right|=1$, (notice $\beta_{i}>0$ according to Theorem 13.3(3) in case $x_{i} \in B_{2}(a+A(P))$ and to the choice of $W_{1}\left(x_{i}\right)$ otherwise) and we let $\alpha_{i}>0$ be small enough for

$$
5 \alpha_{i}+\frac{\alpha_{i}}{\beta_{i}}<c_{i} \eta
$$

where $c_{i}=c\left(W_{1}\left(x_{i}\right), W_{2}\left(x_{i}\right), T_{x_{i}} M_{r}\right)$ as in Lemma 14.1. Next for each $i=1, \ldots, \kappa$ we select $0<\rho_{i}<\rho_{0}$ sufficiently small for

$$
\begin{gathered}
(a+A(P)) \cap \mathbf{B}\left(x_{i}, \rho_{i}\right)=\left(x_{i}+W_{1}\left(x_{i}\right)\right) \cup\left(x_{i}+W_{2}\left(x_{i}\right)\right) \cap \mathbf{B}\left(x_{i}, \rho_{i}\right), \\
M_{r} \cap \mathbf{B}\left(x_{i}, 2 \rho_{i}\right) \subseteq \mathbf{B}\left(x_{i}+T_{x_{i}} M_{r}, \eta \rho_{i} / 2\right)
\end{gathered}
$$

and, for every $x \in \mathbf{B}\left(x_{i}, \rho_{i}\right)$,

$$
\begin{gathered}
\operatorname{dim} W_{1}\left(x_{i}\right) \cap T_{x} M_{r}=1 \\
\operatorname{dim} W_{2}\left(x_{i}\right) \cap T_{x} M_{r}=1 \\
\operatorname{dim} W_{1}\left(x_{i}\right) \cap T_{x} M_{r} \cap W(\pi(x))=0 \\
\operatorname{dim} W_{1}\left(x_{i}\right) \cap W_{2}\left(x_{i}\right) \cap T_{x} M_{r}=0
\end{gathered}
$$

(remember that $\operatorname{dim} W_{1}\left(x_{i}\right) \cap W_{2}\left(x_{i}\right) \cap T_{x_{i}} M_{r}=0$ according to Theorem 13.3(3)) and

$$
\begin{gathered}
\operatorname{dist}\left(T_{x} M_{r}, T_{x_{i}} M_{r}\right)<\alpha_{i} \\
\operatorname{dist}\left(W(\pi(x)), W\left(\pi\left(x_{i}\right)\right)\right)<\alpha_{i} .
\end{gathered}
$$

We let $h_{x_{i}}, S_{i}^{*}$ and $V_{i}$ be associated with $W_{1}\left(x_{i}\right), W_{2}\left(x_{i}\right)$ and $T_{x_{i}} M_{r}$ in Lemma 14.1 in case $x_{i} \in B_{2}(a+A(P))$, and $S_{i}^{*}$ modified according to Remark 14.2 in case $x_{i} \in B_{1}(a+$ $A(P))$. We also put $h_{b}=h_{x_{\kappa}} \circ \cdots \circ h_{x_{1}}$ and $h=h_{b} \circ h_{a} \circ h_{A}$. That conclusion (F) of our 
theorem is satisfied should now be obvious. For further reference we notice that in fact $h$ is $3 \varepsilon / 4$-homotopic to the identity of $U$.

We define $Q$ as follows.

$$
Q=\left((a+A(P)) \backslash\left(\cup_{i=1}^{\kappa} \mathbf{B}\left(x_{i}, \rho_{i}\right)\right)\right) \cup\left(\cup_{i=1}^{\kappa} S_{i}^{*}\right) .
$$

Noticing that $\left(h_{a} \circ h_{A}\right)(P)=a+A(P)$ we infer from Lemma 14.1(E) that

$$
h(P)=h_{b}(a+A(P)) \subseteq\left[(a+A(P)) \backslash \cup_{i=1}^{\kappa} V_{i}\right] \cup\left[\cup_{i=1}^{\kappa} h_{x_{i}}\left((a+A(P)) \cap V_{i}\right)\right] .
$$

The inclusion $h(P) \subseteq Q$ now becomes a consequence of Lemma 14.1(F1,F3). Next we infer from the relation $h\left(P \cap \mathbf{B}\left(x_{i}, \rho_{i}\right)\right)=h(P) \cap \mathbf{B}\left(x_{i}, \rho_{i}\right), i=1, \ldots, \kappa,(53)$ and Lemma 14.1(F2) that ${ }^{(18)}$

$$
\begin{aligned}
\mathscr{H}^{2}(Q \backslash h(P)) & =\mathscr{H}^{2}\left(Q \backslash h(P) \cap\left(\cup_{i=1}^{\kappa} \mathbf{B}\left(x_{i}, \rho_{i}\right)\right)\right) \\
& =\sum_{i=1}^{\kappa} \mathscr{H}^{2}\left(Q \cap \mathbf{B}\left(x_{i}, \rho_{i}\right) \backslash h\left(P \cap \mathbf{B}\left(x_{i}, \rho_{i}\right)\right)\right) \\
& \leqslant \sum_{i=1}^{\kappa} \mathscr{H}^{2}\left(S_{i}^{*} \cap \mathbf{B}\left(x_{i}, \rho_{i}\right) \backslash h_{x_{i}}\left(S_{i} \cap \mathbf{B}\left(x_{i}, \rho_{i}\right)\right)\right)<\frac{\varepsilon}{2} .
\end{aligned}
$$

This proves conclusion $(\mathrm{G})$.

In order to prove conclusion $(\mathrm{H})$ we notice that, referring to Lemma 14.1(F4) and Remark 10.5:

$$
\begin{aligned}
\operatorname{dist}_{\mathscr{H}}(P, Q) \leqslant \operatorname{dist}_{\mathscr{H}}(P, h(P))+\operatorname{dist}_{\mathscr{H}}(h(P), Q) & \\
& <3 \varepsilon / 4+\max \left\{2 \rho_{i}: i=1, \ldots, \kappa\right\}<\varepsilon .
\end{aligned}
$$

With regard to conclusion (I) we observe that

$$
\mathscr{H}^{2}(h(P) \triangle(a+A(P)))=\mathscr{H}^{2}\left(h_{b}(a+A(P)) \triangle(a+A(P))\right)
$$

which equals, according to the definition of $h_{b}$ and Lemma 14.1(E),

$$
=\sum_{i=1}^{\kappa} \mathscr{H}^{2}\left(V_{i} \cap\left(h_{x_{i}}(a+A(P)) \triangle(a+A(P))\right)\right)
$$

which, according to (53), is equal to

$$
\begin{aligned}
& =\sum_{i=1}^{\kappa} \mathscr{H}^{2}\left(h_{x_{i}}\left(S_{i} \cap V_{i}\right) \triangle\left(S_{i} \cap V_{i}\right)\right) \\
& \leqslant \sum_{i=1}^{\kappa} \mathscr{H}^{2}\left(h_{x_{i}}\left(S_{i} \cap V_{i}\right)\right)+\mathscr{H}^{2}\left(\left(S_{i} \cap V_{i}\right)\right)
\end{aligned}
$$

(18) Here $S_{i}$ is associated with $x_{i}, W_{1}\left(x_{i}\right)$ and $W_{2}\left(x_{i}\right)$ as in Lemma 14.1(F). 
which, according to Lemma 14.1(F1) and (F5), is bounded by

$$
\begin{aligned}
& \leqslant 3 \sum_{i=1}^{\kappa} \mathscr{H}^{2}\left(S_{i} \cap V_{i}\right) \\
& \leqslant 3 \sum_{i=1}^{\kappa} \mathscr{H}^{2}\left(S_{i} \cap \mathbf{B}\left(x_{i}, \rho_{i}\right)\right) \\
& =3 \sum_{i=1}^{\kappa} 2 \pi \rho_{i}^{2}<\varepsilon / 2
\end{aligned}
$$

thanks to our choice of $\rho_{0}$. Conclusion (J) is an immediate consequence of (61) and (62).

Since $P$ is $\mathscr{H}^{2}$ essential so is $a+A(P)$. The reader is invited to check this implies that $(a+A(P)) \backslash \cup_{i=1}^{\kappa} \mathbf{B}\left(x_{i}, \rho_{i}\right)$ is $\mathscr{H}^{2}$ essential as well. Since the $S_{i}^{*}, i=1, \ldots, \kappa$, are also $\mathscr{H}^{2}$ essential (Lemma 14.1(F6)), conclusion (K) follows from the definition of $Q$.

In order to prove conclusion (L) we let $\hat{V}_{i}, i=1, \ldots, \kappa$, be as in Lemma 14.1(F7). Let $x \in Q$. We infer from (53) and Lemma 14.1(F7) that if $x \notin \cup_{i=1}^{\kappa}$ Clos $\hat{V}_{i}$ then $Q$ and $a+A(P)$ coincide in a neighborhood of $x$, and if $x \in \operatorname{Clos} V_{i}$ for some $i=1, \ldots, \kappa$ then $Q$ and $S_{i}^{*}$ coincide in a neighborhood of $x$. In both cases it follows that $Q$ is $(\overline{\mathbf{H}}, 1)$ locally connected at $x$.

We now turn to proving conclusion (M). We put $\delta=\min \left\{\eta \rho_{i} / 2: i=1, \ldots, \kappa\right\}$. Assume that $y \in \mathbf{U}\left(x_{i}, \rho_{i}\right) \cap \mathbf{U}\left(M_{r}, \delta\right)$ for some $i=1, \ldots, \kappa$. Choose $x \in M_{r}$ such that $|y-x|<\delta$ and notice that $\left|x-x_{i}\right| \leqslant|x-y|+\left|y-x_{i}\right| \leqslant \delta+\rho_{i} \leqslant 2 \rho_{i}$. Therefore it follows from (54) that $\operatorname{dist}\left(x, x_{i}+T_{x_{i}} M_{r}\right)<\eta \rho_{i} / 2$, and in turn that $\operatorname{dist}\left(y, T_{x_{i}} M_{r}\right)<\eta \rho_{i}$. From this observation and the definition of $Q$ we infer that

$$
Q \cap \mathbf{U}\left(M_{r}, \delta\right) \cap \mathbf{U}\left(x_{i}, \rho_{i}\right) \subseteq S_{i}^{*} \cap \mathbf{U}\left(x_{i}+T_{x_{i}} M_{r}, \eta \rho_{i}\right) \cap \mathbf{U}\left(x_{i}, \rho_{i}\right)
$$

But then, referring to Lemma 14.1(F7), we obtain ${ }^{(19)}$

$$
\begin{aligned}
Q \cap \mathbf{U}\left(M_{r}, \delta\right)=((a+A(P)) \backslash & \left.\cup_{i=1}^{\kappa} \operatorname{Clos} \hat{V}_{i}\right) \\
& \cup\left(\cup_{i=1}^{\kappa} S_{i}^{*} \cap \mathbf{U}\left(x_{i}+T_{x_{i}} M_{r}, \eta \rho_{i}\right) \cap \mathbf{U}\left(x_{i}, \rho_{i}\right)\right) .
\end{aligned}
$$

Finally $M_{r} \cap \mathbf{U}\left(M_{r}, \delta\right)$ is 2-dimensional polyhedral because every set in the finite union above is 2-dimensional polyhedral (recall Lemma 14.1(F8)).

Now let $x \in M_{r} \cap Q$. Referring to Theorem 13.3(1), Lemma 14.1(F9), (63) and the corresponding footnote, we conclude that $\mathscr{W}_{Q}(x) \leqslant 2$. If $x \notin \cup_{i=1}^{\kappa} \operatorname{Clos} \hat{V}_{i}$ then $a+A(P)$ and $Q$ coincide in a neighborhood of $x$ as follows from conclusions (E) and (F7) of Lemma 14.1, so that conclusions (M2) and (M4) of the present theorem follow from Theorem 13.3, respectively conclusions (2) and (3), whereas our present conclusion (M3) follows from the fact that $x \notin B(a+A(P))$ whence also $x \notin B(Q)$.

Finally assume that $x \in \operatorname{Clos} \hat{V}_{i}$ for some $i=1, \ldots, \kappa$. Then every $W \in \mathscr{W}_{Q}(x)$ satisfies one of the conditions (a), (b) and (c) listed in Lemma 14.1(F9). In order to conclude that the present conclusion (M2) holds true we refer to (55) in case $W$ satisfies (a), we refer to (58) in case $W$ satisfies (b), and we refer to (56) in case $W$ verifies (c). In order to show that our

(19) Even though this is the finite union of sets which are not pairwise disjoint, any two of them with nonempty intersection coincide on their intersection.

$4^{\mathrm{e}}$ SÉRIE - TOME $42-2009-\mathrm{N}^{\mathrm{o}} 1$ 
conclusion (M4) holds we proceed in the following way. Denote by $W_{1}^{\prime}$ and $W_{2}^{\prime}$ the elements of $\mathscr{W}_{Q}(x)$. If these verify conditions (a) and (c) of Lemma 14.1(F9) then we refer to (58) to conclude that $\operatorname{dim} W_{1}^{\prime} \cap W_{2}^{\prime} \cap T_{x} M_{r}=0$. We next assume that $W_{1}^{\prime}, W_{2}^{\prime}$ verify either conditions (a) and (b) or conditions (b) and (c). Notice that in both cases necessarily $W_{1}\left(x_{i}\right) \cap W_{2}\left(x_{i}\right) \subseteq$ $W_{1}^{\prime} \cap W_{2}^{\prime}$. Assume if possible that $\operatorname{dim} W_{1}^{\prime} \cap W_{2}^{\prime} \cap T_{x} M_{r}=1$ (recall (M2) to infer that this dimension is either 0 or 1$)$, then $W_{1}^{\prime} \cap W_{2}^{\prime} \subseteq T_{x} M_{r}$ and in turn $W_{1}\left(x_{i}\right) \cap W_{2}\left(x_{i}\right) \subseteq T_{x} M_{r}$ in contradiction with (58).

It remains to show that our conclusion (M3) holds at $x$. Let $W \in \mathscr{W}_{Q}(x)$. If $W$ verifies condition (a) of Lemma 14.1(F9) then we refer to (57). If $W$ verifies condition (c) then $x \in$ $x_{i}+W_{2}\left(x_{i}\right)$, whence $x \in a+A(P)$ and $W \in \mathscr{W}_{a+A(P)}(x)$ in view of (53). Since also $x \neq x_{i}$ we see that $x \notin B(a+A(P))$ and therefore $\operatorname{dim} W \cap T_{x} M_{r} \cap W(\pi(x))=0$. The last case to be covered is when $W$ verifies condition (b) of Lemma 14.1(F9). Remember we need to establish that $\operatorname{dim} W \cap T_{x} M_{r} \cap W(\pi(x))=0$. If not then necessarily $\operatorname{dim} W \cap T_{x_{i}} M_{r}=1$ (in view of (M2)) and $\operatorname{dist}\left(W, T_{x_{i}} M_{r}\right) \geqslant \beta_{i}$, by the definition of $\beta_{i}$, because $W_{1}\left(x_{i}\right) \cap W_{2}\left(x_{i}\right) \subseteq$ $W$ (according to Lemma 14.1(F9)(b)). We will use this together with the estimate stated in Lemma 14.1(F9)(b) to infer that

$$
\operatorname{dim} W \cap T_{x} M_{r} \cap W(\pi(x))=0 .
$$

Indeed,

$$
\begin{aligned}
\operatorname{dist}\left(W \cap T_{x_{i}} M_{r}, W_{2}\left(x_{i}\right) \cap T_{x_{i}} M_{r}\right) \leqslant & \operatorname{dist}\left(W \cap T_{x_{i}} M_{r}, W \cap T_{x} M_{r}\right) \\
& +\operatorname{dist}\left(W \cap T_{x} M_{r}, W_{2}\left(x_{i}\right) \cap T_{x_{i}} M_{r}\right) .
\end{aligned}
$$

Notice that $W_{2}\left(x_{i}\right) \cap T_{x_{i}} M_{r}=W\left(\pi\left(x_{i}\right)\right) \cap T_{x_{i}} M_{r}$ because $\operatorname{dim} W_{2}\left(x_{i}\right) \cap W\left(\pi\left(x_{i}\right)\right) \cap$ $T_{x_{i}} M_{r}=1$ (since $x_{i} \in B(a+A(P))$ ), $W_{2}\left(x_{i}\right) \neq T_{x_{i}} M_{r}$ (according to Theorem 13.3(3)) and $W\left(\pi\left(x_{i}\right)\right) \neq T_{x_{i}} M_{r}{ }^{(20)}$. Assume if possible that $\operatorname{dim} W \cap T_{x} M_{r} \cap W(\pi(x))=1$. Then $W \cap T_{x} M_{r}=W(\pi(x)) \cap T_{x} M_{r}$. Inequality (64) together with Lemma 8.5 and (59), (60) would yield

$$
\begin{aligned}
\operatorname{dist}\left(W \cap T_{x_{i}} M_{r}, W_{2}\left(x_{i}\right) \cap T_{x_{i}} M_{r}\right) \leqslant & \operatorname{dist}\left(W \cap T_{x_{i}} M_{r}, W \cap T_{x} M_{r}\right) \\
& +\operatorname{dist}\left(W(\pi(x)) \cap T_{x} M_{r}, W\left(\pi\left(x_{i}\right)\right) \cap T_{x_{i}} M_{r}\right) \\
\leqslant & \operatorname{dist}\left(T_{x_{i}} M_{r}, T_{x} M_{r}\right)\left(1+\frac{1}{\operatorname{dist}\left(W, T_{x_{i}} M_{r}\right)}\right) \\
& +2 \operatorname{dist}\left(W(\pi(x)), W\left(\pi\left(x_{i}\right)\right)\right) \\
& +2 \operatorname{dist}\left(T_{x} M_{r}, T_{x_{i}} M_{r}\right) \\
\leqslant & \alpha_{i}\left(1+\frac{1}{\beta_{i}}\right)+4 \alpha_{i} \leqslant c_{i} \eta
\end{aligned}
$$

contradicting Lemma 14.1(F9)(b). This completes the proof of the theorem.

(20) Observe that $T_{y} M_{r} \neq W(\pi(y))$ for every $y \in \mathbf{B}(B, R)$, in fact $\operatorname{dist}\left(T_{y} M_{r}, W(\pi(y))\right)=1$ because $\nabla d(y) \in$ $\left(T_{y} M_{r}\right)^{\perp} \cap W(\pi(y))$. 


\section{Local connectedness with respect to integral currents homology}

\subsection{Theorem. - Assume that}

(A) $\kappa=1,2, \ldots$ is an integer, $\iota_{k} \in\{1,2\}, k=1, \ldots, \kappa, Y_{k, i} \subseteq \mathbb{R}^{3}, k=1, \ldots, \kappa, i=$ $1, \ldots, \iota_{k}$, are Borel measurable;

(B) For every $k=1, \ldots, \kappa$ such that $\iota_{k}=2$ one has

$$
Y_{k, 1} \cap \operatorname{Clos} Y_{k, 2}=Y_{k, 1} \cap Y_{k, 2} ;
$$

(C) There exists $Z \subseteq \mathbb{R}^{n}$ such that $Z \subseteq Y_{k, i}$ for every $k$, $i$, and

$$
Z=Y_{k_{1}, i_{1}} \cap Y_{k_{2}, i_{2}}=\operatorname{Clos} Y_{k_{1}, i_{1}} \cap \operatorname{Clos} Y_{k_{2}, i_{2}}
$$

whenever $k_{1}, k_{2}=1, \ldots, \kappa, i_{1}=1, \ldots, \iota_{k_{1}}, i_{2}=1, \ldots, \iota_{k_{2}}$, and $k_{1} \neq k_{2}$;

(D) $y_{0} \in Z$ and for every $k=1, \ldots, \kappa$ and $i=1, \ldots, \iota_{k}$ there exists a Lipschitzian

$$
H_{k, i}:[0,1] \times Y_{k, i} \rightarrow Y_{k, i}
$$

such that $H_{k, i}(0, y)=y$ and $H_{k, i}(1, y)=y_{0}$ for every $y \in Y_{k, i}$;

(E) For every $k_{1}, k_{2}=1, \ldots, \kappa$ and $i_{1}=1, \ldots, \iota_{k_{1}}, i_{2}=1, \ldots, \iota_{k_{2}}$

$$
H_{k_{1}, i_{1}} \uparrow\left([0,1] \times Y_{k_{1}, i_{1}} \cap Y_{k_{2}, i_{2}}\right)=H_{k_{2}, i_{2}} \uparrow\left([0,1] \times Y_{k_{1}, i_{1}} \cap Y_{k_{2}, i_{2}}\right) .
$$

Then

$$
\overline{\mathbf{H}}_{q}(Y)=\{0\} \text { (reduced integral currents homology) }
$$

for $q=0,1$, where $Y=\cup_{k=1}^{\kappa} \cup_{i=1}^{\iota_{k}} Y_{k, i}$.

15.2. Remark. - It follows from hypothesis (E) that a map

$$
H:[0,1] \times Y \rightarrow Y
$$

is well-defined by $H \uparrow\left([0,1] \times Y_{k, i}\right)=H_{k, i}$. Obviously $H(0, y)=y$ and $H(1, y)=y_{0}$ for every $y \in Y$, according to (D). Moreover one readily checks that $H$ is continuous, therefore $Y$ is (continuously) contractible to a point. As a consequence $H_{q}(Y ; \mathbb{Z})=\{0\}$ (reduced singular homology) for every $q=0,1,2, \ldots$ The point here is that the continuous homotopy $H$ does not need to be Lischitzian - the Lispchitz condition is not preserved on gluing together the pieces $H_{k, i}$. The homological triviality of $Y$ in the Lipschitzian category will be obtained in a somewhat more pedestrian way than in the singular case. Note also that a "Mayer-Vietoris" argument does not apply since the pieces $Y_{k, i}$ are not assumed to be relatively open in $Y$.

Proof. - We start with the case $q=0$. Let $0 \neq T \in \mathbf{I}_{0}(Y)$ be such that $\langle T, 1\rangle=0$. We need to show that $T=\partial S$ for some $S \in \mathbf{I}_{1}(Y)$. There exist a positive integer $m$ and $a_{1}, \ldots, a_{m}, b_{1}, \ldots, b_{m} \in Y$ such that

$$
T=\sum_{j=1}^{m}\left(\boldsymbol{\delta}_{b_{j}}-\boldsymbol{\delta}_{a_{j}}\right)
$$

Fix $j=1, \ldots, m$ and choose $k_{j}, k_{j}^{\prime}$ and $i_{j}, i_{j}^{\prime}$ such that $a_{j} \in Y_{k_{j}, i_{j}}$ and $b_{j} \in Y_{k_{j}^{\prime}, i_{j}^{\prime}}$. We define

$$
f_{j}:[0,1] \rightarrow \mathbb{R}^{n}: t \mapsto \begin{cases}H_{k_{j}, i_{j}}\left(2 t, a_{j}\right) & \text { if } 0 \leqslant t \leqslant 1 / 2 \\ H_{k_{j}^{\prime}, i_{j}^{\prime}}\left(2-2 t, b_{j}\right) & \text { if } 1 / 2 \leqslant t \leqslant 1 .\end{cases}
$$

$4^{\text {e }}$ SÉRIE - TOME $42-2009-\mathrm{N}^{\mathrm{o}} 1$ 
We readily check that $\operatorname{Lip} f_{j}<\infty, \operatorname{im} f_{j} \subseteq Y, f_{j}(0)=a_{j}$ and $f_{j}(1)=b_{j}$. Letting $S_{j}=$ $f_{j \#} \llbracket 0,1 \rrbracket$ we obtain $S_{j} \in \mathbf{I}_{1}(Y)$ and $\partial S_{j}=\boldsymbol{\delta}_{b_{j}}-\boldsymbol{\delta}_{a_{j}}$. Setting $S=\sum_{j=1}^{m} S_{j}$ completes the proof in case $q=0$.

Next we consider the case $q=1$. Let $0 \neq T \in \mathbf{I}_{1}(Y)$ be such that $\partial T=0$. We need to show that $T=\partial S$ for some $S \in \mathbf{I}_{2}(Y)$. According to [11, 4.2.25] there exist $m \in \mathbb{Z}^{+} \cup\{\infty\}$ and Lipschitzian functions $f_{j}:[0,1] \rightarrow Y, j=1,2, \ldots, m$, such that $f_{j}\llcorner[0,1)$ is injective, $f_{j}(0)=f_{j}(1)$, and

$$
\begin{gathered}
\sum_{j=1}^{m} \mathbf{M}\left(f_{j \#} \llbracket 0,1 \rrbracket\right)=\mathbf{M}(T)<\infty \\
T=\sum_{j=1}^{m} f_{j \#} \llbracket 0,1 \rrbracket \text { (weakly). }
\end{gathered}
$$

Therefore the claim will be proved if we show that for each $j=1,2, \ldots, m$ there exists

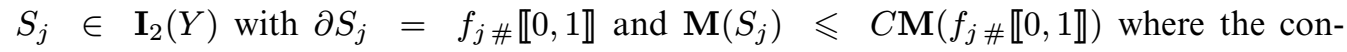
stant $C$ does not depend on $j$. We fix $j=1, \ldots, m$ and from now on we drop the index $j$ in order to keep the notation short. We start by defining a partition of $[0,1]$, say $\left\{A_{k, i}: k=1, \ldots, \kappa\right.$ and $\left.i=1, \ldots, \iota_{k}\right\}$, such that each $A_{k, i}$ is Borel measurable and $f\left(\operatorname{Clos} A_{k, i}\right) \subseteq Y_{k, i}$. $\mathbb{P}$ Letting $A_{k, i}^{\prime}=f^{-1}\left(Y_{k, i}\right)$ we notice that we can define a Borel measurable partition of $[0,1]$ whose members are subsets of $A_{k, i}^{\prime}$. Thus it suffices to establish that $f\left(\operatorname{Clos} A_{k, i}^{\prime}\right) \subseteq Y_{k, i}$. Let $s \in[0,1]$ and $s_{j} \in A_{k, i}^{\prime}, j=1,2, \ldots$, such that $s_{j} \rightarrow s$ as $j \rightarrow \infty$. Since $f(s) \in Y$ there are $k^{\prime}$ and $i^{\prime}$ such that $f(s) \in Y_{k^{\prime}, i^{\prime}}$. Notice also that $f(s) \in \operatorname{Clos} Y_{k, i}$ since $f$ is continuous. If $k=k^{\prime}$ then $f(s) \in Y_{k, i}$ according to hypothesis (B). If $k \neq k^{\prime}$ then $f(s) \in Z \subseteq Y_{k, i}$ according to hypothesis (C). Q Referring to [9, 3.1.1 Theorem 1], we define Lipschitzian maps

$$
G_{k, i}: \mathbb{R}^{2} \rightarrow \mathbb{R}^{3}
$$

such that $G_{k, i}(t, s)=H_{k, i}(t, f(s))$ whenever $(t, s) \in[0,1] \times \operatorname{Clos} A_{k, i}$. Letting $R_{k, i}=$ $\mathbf{E}^{1}\left\llcorner A_{k, i}{ }^{(21)}\right.$ we also define

$$
S_{k, i}=G_{k, i \#}\left(\llbracket 0,1 \rrbracket \times R_{k, i}\right)
$$

and

$$
S=\sum_{k=1}^{\kappa} \sum_{i=1}^{\iota_{k}} S_{k, i}
$$

Clearly $S \in \mathscr{R}_{2}\left(\mathbb{R}^{3}\right)$ and spt $S \subseteq Y$. We notice that

$$
\partial S_{k, i}=G_{k, i \#}\left(\boldsymbol{\delta}_{1} \times R_{k, i}-\boldsymbol{\delta}_{0} \times R_{k, i}-\llbracket 0,1 \rrbracket \times \partial R_{k, i}\right) .
$$

Observe that

$$
\mathscr{H}^{1}\left(\operatorname{spt} G_{k, i \#}\left(\boldsymbol{\delta}_{0} \times R_{k, i}\right)\right) \leqslant \mathscr{H}^{1}\left(G_{k, i}\left(\{0\} \times \operatorname{Clos} A_{k, i}\right)\right) \leqslant \mathscr{H}^{1}\left(\left\{x_{0}\right\}\right)=0 .
$$

Since $G_{k, i \#}\left(\boldsymbol{\delta}_{0} \times R_{k, i}\right) \in \mathscr{R}_{1}\left(\mathbb{R}^{3}\right)$ we infer that

$$
G_{k, i \#}\left(\boldsymbol{\delta}_{0} \times R_{k, i}\right)=0 .
$$

$\overline{(21) \text { Recall that } \mathbf{E}^{1}}=\mathscr{L}^{1} \wedge \mathbf{e}_{1}$ is the 1-dimensional current in $\mathbb{R}$ corresponding to Lebesgue integration. 
We also observe that if $(t, s) \in \operatorname{spt} \boldsymbol{\delta}_{1} \times R_{k, i}=\{1\} \times \operatorname{Clos} A_{k, i}$ then $G_{k, i}(t, s)=$ $H_{k, i}(1, f(s))=f(s)$. Letting $F(t, s)=f(s),(t, s) \in[0,1] \times[0,1]$, we infer from [11, 4.1.15] that

$$
G_{k, i \#}\left(\boldsymbol{\delta}_{1} \times R_{k, i}\right)=F_{\#}\left(\boldsymbol{\delta}_{1} \times R_{k, i}\right)=f_{\#} R_{k, i} .
$$

Plugging (66) and (67) into (65) yields

$$
\begin{aligned}
\partial S & =\sum_{k=1}^{\kappa} \sum_{i=1}^{\iota_{k}}\left(f_{\#} R_{k, i}-G_{k, i \#}\left(\llbracket 0,1 \rrbracket \times \partial R_{k, i}\right)\right) \\
& =f_{\#} \llbracket 0,1 \rrbracket-\sum_{k=1}^{\kappa} \sum_{i=1}^{\iota_{k}} G_{k, i \#}\left(\llbracket 0,1 \rrbracket \times \partial R_{k, i}\right) .
\end{aligned}
$$

We now intend to show that

$$
\sum_{k=1}^{\kappa} \sum_{i=1}^{\iota_{k}} G_{k, i \#}\left(\llbracket 0,1 \rrbracket \times \partial R_{k, i}\right)=0
$$

so that $T=\partial S$ (and consequently $S \in \mathbf{I}_{2}(Y)$ ). Consider $k=1, \ldots, \kappa$ such that $\iota_{k}=2$ (if any exists). We observe that if $s \in[0,1]$ and $f(s) \in Y_{k, 2} \backslash Y_{k, 1}$ then $s \in \operatorname{Int} A_{k, 2}{ }^{(22)}$. $\mathbb{P}$ For if not the pigeonhole principle would ensure the existence of $\left(k^{\prime}, i^{\prime}\right) \neq(k, 2)$ and $s_{j} \in A_{k^{\prime}, i^{\prime}}$, $j=1,2, \ldots$, such that $s_{j} \rightarrow s$ as $j \rightarrow \infty$. Note that $f(s) \in f\left(\operatorname{Clos} A_{k^{\prime}, i^{\prime}}\right) \subseteq Y_{k^{\prime}, i^{\prime}}$. If $k=k^{\prime}$ then necessarily $i^{\prime}=1$, in contradiction with $f(s) \notin Y_{k, 1}$. Thus $k \neq k^{\prime}$ and referring to hypothesis (C) we infer that $f(s) \in Y_{k^{\prime}, i^{\prime}} \cap Y_{k, 2}=Z \subseteq Y_{k, 1}$, a contradiction. Q Therefore if $s \in \operatorname{spt} \partial R_{k, 2} \subseteq$ Bdry $A_{k, 2}$ then $f(s) \in Y_{k, 1} \cap Y_{k, 2}$. It follows from hypothesis (E) that $G_{k, 1}(t, s)=G_{k, 2}(t, s)$ whenever $(t, s) \in \operatorname{spt}\left(\llbracket 0,1 \rrbracket \times \partial R_{k, 2}\right)$. Since $\llbracket 0,1 \rrbracket \times \partial R_{k, 2} \in \mathbf{F}_{1}\left(\mathbb{R}^{3}\right)$ we infer from [11, 4.1.15] that

$$
\sum_{i=1}^{2} G_{k, i \#}\left(\llbracket 0,1 \rrbracket \times \partial R_{k, i}\right)=G_{k, 1 \#}\left(\llbracket 0,1 \rrbracket \times \partial\left(\sum_{i=1}^{2} R_{k, i}\right)\right) .
$$

An argument similar to that given above, based on hypothesis (C), shows that if $s \in[0,1]$ and $f(s) \in\left(\cup_{i=1}^{\iota_{k}} Y_{k, i}\right) \backslash Z$ then $s \in \operatorname{Int} \cup_{i=1}^{\iota_{k}} A_{k, i}$. Consequently if $s \in \operatorname{spt}\left(\partial \sum_{i=1}^{\iota_{k}} R_{k, i}\right) \subseteq$ Bdry $\cup_{i=1}^{\iota_{k}} A_{k, i}$ then $f(s) \in Z$. Therefore $G_{1,1}(t, s)=G_{k, 1}(t, s)$ whenever $k=1, \ldots, \kappa$ and

$$
(t, s) \in \operatorname{spt}\left(\llbracket 0,1 \rrbracket \times \partial\left(\sum_{i=1}^{\iota_{k}} R_{k, i}\right)\right),
$$

according to hypotheses (C) and (E). It follows from (70) that

$$
\begin{aligned}
\sum_{k=1}^{\kappa} \sum_{i=1}^{\iota_{k}} G_{k, i \#}\left(\llbracket 0,1 \rrbracket \times \partial R_{k, i}\right) & =\sum_{k=1}^{\kappa} G_{k, 1 \#}\left(\llbracket 0,1 \rrbracket \times \partial\left(\sum_{i=1}^{\iota_{k}} R_{k, i}\right)\right) \\
& =\sum_{k=1}^{\kappa} G_{1,1 \#}\left(\llbracket 0,1 \rrbracket \times \partial\left(\sum_{i=1}^{\iota_{k}} R_{k, i}\right)\right) \\
& =G_{1,1 \#}\left(\llbracket 0,1 \rrbracket \times \boldsymbol{\delta}_{1}\right)-G_{1,1 \#}\left(\llbracket 0,1 \rrbracket \times \boldsymbol{\delta}_{0}\right)=0
\end{aligned}
$$

because $f(1)=f(0)$. This completes the proof of (74).

(22) Interior relative to the quotient topology of $[0,1]$ where 0 and 1 have been identified.

$4^{\mathrm{e}}$ SÉRIE - TOME $42-2009-\mathrm{N}^{\mathrm{o}} 1$ 
To finish the proof of the case $q=1$ we need to establish that

$$
\mathbf{M}(S) \leqslant C \mathbf{M}(T) .
$$

Fix $k=1, \ldots, \kappa, i=1, \ldots, \iota_{k}$ and $1 \leqslant \lambda(1)<\lambda(2) \leqslant 3$. An elementary computation shows that

$$
\left|d\left\langle G_{k, i}(t, s), e_{\lambda(1)}\right\rangle \wedge d\left\langle G_{k, i}(t, s), e_{\lambda(2)}\right\rangle\right| \leqslant 2\left(\operatorname{Lip} H_{k, i}\right)^{2}\left|f^{\prime}(s)\right|
$$

whenever $G_{k, i}$ is differentiable at $(t, s)$. Given $\omega_{\lambda} \in \mathscr{D}^{0}\left(\mathbb{R}^{3}\right)$ we obtain

$$
\begin{aligned}
\left|\left\langle S_{k, i}, \omega_{\lambda} d x_{\lambda(1)} \wedge d x_{\lambda(2)}\right\rangle\right| & =\left|\left\langle\llbracket 0,1 \rrbracket \times R_{k, i},\left(\omega_{\lambda} \circ G_{k, i}\right) G_{k, i}^{\#} d x_{\lambda(1)} \wedge d x_{\lambda(2)}\right\rangle\right| \\
& \leqslant \int_{[0,1] \times A_{k, i}} 2 \omega_{\lambda}\left(G_{k, i}(t, s)\right)\left(\operatorname{Lip} H_{k, i}\right)^{2}\left|f^{\prime}(s)\right| d \mathscr{L}^{1}(s) \\
& \leqslant 2\left|\omega_{\lambda}\right|_{\infty}\left(\operatorname{Lip} H_{k, i}\right)^{2} \int_{A_{k, i}}\left|f^{\prime}(s)\right| d \mathscr{L}^{1}(s) .
\end{aligned}
$$

It follows that

$$
\mathbf{M}\left(S_{k, i}\right) \leqslant C_{0}\left(\operatorname{Lip} H_{k, i}\right)^{2} \int_{A_{k, i}}\left|f^{\prime}(s)\right| d \mathscr{L}^{1}(s)
$$

and summing over $k$ and $i$,

$$
\mathbf{M}(S) \leqslant C \int_{0}^{1}\left|f^{\prime}(s)\right| d \mathscr{L}^{1}(s)=C \mathbf{M}\left(f_{\#} \llbracket 0,1 \rrbracket\right)
$$

where

$$
C=C_{0} \max \left\{\left(\operatorname{Lip}, H_{k, i}\right)^{2}: k=1, \ldots, \kappa \text { and } i=1, \ldots, \iota_{k}\right\}
$$

\section{End of the proof}

We are now ready to prove Theorem 6.1 .

Proof. - We let $R>0$ and $\Lambda \geqslant 1$ be associated with $B$ respectively in Theorem 12.1 and Theorem 12.3. We choose $r_{0}>0$ sufficiently small for

$$
\mathscr{H}^{2}\left(X \cap \mathbf{U}_{\infty}\left(B, r_{0}\right)\right)<\frac{\varepsilon}{6 \Lambda^{2}} .
$$

P Since $\mathscr{H}^{2}(X)<\infty$ we notice that

$$
\lim _{j \rightarrow \infty} \mathscr{H}^{2}\left(X \cap \mathbf{U}_{\infty}\left(B, j^{-1}\right)\right)=\mathscr{H}^{2}\left(X \cap\left(\cap_{j=1}^{\infty} \mathbf{U}_{\infty}\left(B, j^{-1}\right)\right)\right)=\mathscr{H}^{2}(B)=0 \text {. Q }
$$

Next we define

$$
\begin{aligned}
r & =\min \left\{\frac{R}{4}, \frac{\varepsilon}{3}, \frac{2 r_{0}}{21}\right\} \\
\varepsilon_{11.4} & =\min \left\{\frac{\varepsilon}{6 \Lambda^{2}}, \frac{r}{2 \sqrt{3}}\right\} \\
\varepsilon_{14.3} & =\min \left\{\frac{\varepsilon}{6 \Lambda^{2}}, \frac{r}{2}\right\} \\
\eta_{11.4} & =2 r+3 \varepsilon_{14.3} .
\end{aligned}
$$

Observe that

$$
\varepsilon_{11.4} \leqslant r \leqslant \eta_{11.4} \leqslant \frac{r_{0}}{3}
$$


Apply Theorem 11.4 to $X, \varepsilon_{11.4}$ and $\eta_{11.4}$ and denote by $U, f_{0}$ and $P$ the open set, Lipschitzian map and polyhedral set provided in the conclusion of that theorem. Next apply Theorem 14.3 to $B, r, P, U$ and $\varepsilon_{14.3}$ as above and denote by $Q$ and $h$ the set and Lipschitzian map appearing in the conclusion of that theorem.

We let $f$ denote an arbitrary extension of $g_{r} \circ h \circ f_{0}{ }^{(23)}$ to $\mathbb{R}^{3}\left(g_{r}\right.$ is as in Theorem 12.3) and $Y=g_{r}(Q)$. Notice that

$$
\begin{aligned}
f(X) & =g_{r}\left(h\left(f_{0}(X)\right)\right) \\
& \subseteq g_{r}(h(P)) \\
& \subseteq g_{r}(Q) \\
& =Y .
\end{aligned}
$$

Thus conclusion (D) is satisfied.

We next notice that

$\operatorname{dist}_{\mathscr{H}}(X, Y) \leqslant \operatorname{dist}_{\mathscr{H}}(X, P)+\operatorname{dist}_{\mathscr{H}}(P, Q)+\operatorname{dist}_{\mathscr{H}}\left(Q, g_{r}(Q)\right)<\varepsilon_{11.4}+\varepsilon_{14.3}+2 r \leqslant \varepsilon$ according respectively to Theorems 11.4(F), 14.3(H) and 12.3(A). Therefore conclusion (E) holds true.

We now turn to proving the measure estimate in conclusion $(\mathrm{F})$. We start by noticing that $g_{r}\left(Q \cap V_{2 r}\right)$ and $g_{r}\left(Q \backslash V_{2 r}\right)$ are disjoint (according to Theorem 12.3(A,C)) and Borel measurable. Furthermore $g_{r}\left(Q \backslash V_{2 r}\right)=Q \backslash V_{2 r}$ (Theorem 12.3(A)). Therefore

$$
\mathscr{H}^{2}(Y)=\mathscr{H}^{2}\left(g_{r}\left(Q \cap V_{2 r}\right)\right)+\mathscr{H}^{2}\left(Q \backslash V_{2 r}\right) .
$$

We now estimate the measure of $Q \cap V_{2 r}$ :

$$
\begin{array}{rlrl}
\mathscr{H}^{2}( & \left.\cap V_{2 r}\right) & \\
\leqslant & \mathscr{H}^{2}\left(h(P) \cap V_{2 r}\right)+\mathscr{H}^{2}(Q \backslash h(P)) & \\
\leqslant & \mathscr{H}^{2}\left(h(P) \cap V_{2 r}\right)+\varepsilon_{14.3} & & (\text { Theorem } 14.3(\mathrm{G})) \\
\leqslant & \mathscr{H}^{2}\left(h\left(P \cap V_{2 r+\varepsilon_{14.3}}\right)\right)+\varepsilon_{14.3} & (*) & \\
\leqslant & \mathscr{H}^{2}\left((a+P) \cap V_{2 r+2 \varepsilon_{14.3}}\right) & & (* *) \\
& \quad+\mathscr{H}^{2}(h(P) \backslash(a+P))+\varepsilon_{14.3} & & \left(|a|<\varepsilon_{14.3} \text { and Theorem } 14.3(1)\right) \\
\leqslant & \mathscr{H}^{2}\left(P \cap V_{2 r+3 \varepsilon_{14.3}}\right)+2 \varepsilon_{14.3} & & \left(\text { By definition of } \eta_{14.3}\right) \\
\leqslant & \mathscr{H}^{2}\left(P \cap \mathbf{U}_{\infty}\left(B, \eta_{14.3}\right)\right)+2 \varepsilon_{14.3} & & \left(\text { By }(72) \text { and the choice of } r_{0}\right) \\
\leqslant & \left.\varepsilon_{11.4}+\mathscr{H}^{2}\left(X \cap \mathbf{U}_{\infty}\left(B, 3 \eta_{14.3}\right)\right)+2 \varepsilon_{14.3}\right) \\
\leqslant & \varepsilon_{11.4}+\frac{\varepsilon}{6 \Lambda^{2}}+2 \varepsilon_{14.3} & & \\
\leqslant & \frac{2 \varepsilon}{3 \Lambda^{2}}, & &
\end{array}
$$

where (*) follows from the inclusion $h(P) \cap V_{2 r} \subseteq h\left(P \cap V_{2 r+\varepsilon_{14.3}}\right)$ P If $y=h(x), x \in P$, then $|y-x|<\varepsilon_{14.3}$ according to Theorem 14.3(F), therefore $\operatorname{dist}(x, B) \leqslant \operatorname{dist}(y, B)+\varepsilon_{14.3}$

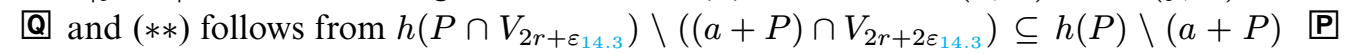
Assume $y=h(x), x \in P \cap V_{2 r+\varepsilon_{14.3}}$ and $y \notin(a+P) \cap V_{2 r+2 \varepsilon_{14.3}}$. Since $|y-x|<\varepsilon_{14.3}$ we

(23) Which is defined on $U$.

$4^{\mathrm{e}}$ SÉRIE - TOME $42-2009-\mathrm{N}^{\mathrm{o}} 1$ 
infer that $\operatorname{dist}(y, B) \leqslant|y-x|+\operatorname{dist}(x, B)<2 r+2 \varepsilon_{14.3}$. Therefore $y \notin a+P$. $Q$ We also observe that

$$
\begin{array}{rlrl}
\mathscr{H}^{2}\left(Q \backslash V_{2 r}\right) & \leqslant \mathscr{H}^{2}(Q) & \\
& \leqslant \varepsilon_{14.3}+\mathscr{H}^{2}(P) & & \text { (Theorem 14.3(J)) } \\
& \leqslant \varepsilon_{14.3}+\varepsilon_{11.4}+\mathscr{H}^{2}(X) & & \text { (Theorem 11.4(H)) } \\
& \leqslant \frac{\varepsilon}{3 \Lambda^{2}} . & &
\end{array}
$$

Plugging the last two inequalities in (73) and referring to Theorem 12.3(B) yields

$$
\begin{aligned}
\mathscr{H}^{2}(Y) & =\mathscr{H}^{2}\left(g_{r}\left(Q \cap V_{2 r}\right)\right)+\mathscr{H}^{2}\left(Q \backslash V_{2 r}\right) \\
& <\Lambda^{2} \mathscr{H}^{2}\left(Q \cap V_{2 r}\right)+\mathscr{H}^{2}\left(Q \backslash V_{2 r}\right) \leqslant \varepsilon .
\end{aligned}
$$

With regard to the reverse inequality we note that

and also,

$$
\begin{aligned}
\mathscr{H}^{2}(Y) & =\mathscr{H}^{2}\left(g_{r}\left(Q \cap V_{2 r}\right)\right)+\mathscr{H}^{2}\left(Q \backslash V_{2 r}\right) \\
& \geqslant \mathscr{H}^{2}\left(Q \backslash V_{2 r}\right) \\
& =\mathscr{H}^{2}(Q)-\mathscr{H}^{2}\left(Q \cap V_{2 r}\right) \\
& >\mathscr{H}^{2}(Q)-\frac{2 \varepsilon}{3 \Lambda^{2}}
\end{aligned}
$$

$$
\begin{array}{rlrl}
\mathscr{H}^{2}(Q) & \geqslant \mathscr{H}^{2}(h(P)) & & \text { (Theorem 14.3(G)) } \\
& \geqslant \mathscr{H}^{2}(a+P)-\varepsilon_{14.3} & & \text { (Theorem 14.3(I)) } \\
& =\mathscr{H}^{2}(P)-\varepsilon_{14.3} & & \\
& >\mathscr{H}^{2}(X)-\varepsilon_{14.3}-\varepsilon_{11.4} & & \text { (Theorem 11.4(H)) } \\
& \geqslant \mathscr{H}^{2}(X)-\frac{\varepsilon}{3 \Lambda^{2}} . &
\end{array}
$$

Now clearly

$$
\mathscr{H}^{2}(Y)>\mathscr{H}^{2}(X)-\varepsilon
$$

and the proof of conclusion (F) is complete.

In order to establish that $Y$ is $\mathscr{H}^{2}$ essential (conclusion $(\mathrm{G})$ ) we simply notice that $g_{r}$ is a $C^{1}$ diffeomorphism of $\mathbb{R}^{3} \backslash V_{r}$ onto $\mathbb{R}^{3} \backslash B$ (Theorem 12.3(C)) - so that $g_{r}\left(Q \backslash V_{r}\right)$ is itself $\mathscr{H}^{2}$ essential because so is $Q$ according to Theorem $14.3(\mathrm{~K})-$, and that $\mathscr{H}^{2}\left(g_{r}\left(V_{r}\right)\right)=$ $\mathscr{H}^{2}(B)=0$.

We now show that conclusion (I) holds true. Since $f_{0}$ is $\varepsilon_{11.4} \sqrt{3}$-homotopic to id $\operatorname{in}_{U}$ in the Lipschitzian category (Theorem $11.4(\mathrm{~J})$ ), and $h$ is $\varepsilon_{14.3}$-homotopic to $\operatorname{id}_{U}$ in the Lipschitzian category (Theorem 14.3(F)) we infer that $h \circ f_{0}$ is $\varepsilon_{14.3}+\varepsilon_{11.4} \sqrt{3}$-homotopic to id in $_{U}$ the Lipschitzian category (Remark 10.7). In other words there exists a Lipschitzian map $H$ : $[0,1] \times U \rightarrow U$ such that $H(0, x)=x, H(1, x)=h\left(f_{0}(x)\right)$ and $|x-H(t, x)|<\varepsilon_{14.3}+$ $\varepsilon_{11.4} \sqrt{3} \leqslant r$ whenever $x \in U$ and $0 \leqslant t \leqslant 1$. Since $H(t, x) \in V_{r}$ we have $g_{r}(H(t, x))=$ $\pi(H(x, t)) \in B$ for every $x \in B$ and $0 \leqslant t \leqslant 1$ according to Theorem 12.3(A). In particular $f(B) \subseteq B$. We define $H^{\prime}:[0,1] \times B \rightarrow B$ by $H^{\prime}(t, x)=g_{r}(H(t, x))$ and we notice $H^{\prime}$ witnesses the fact that the restriction $f: B \rightarrow B$ and $\operatorname{id}_{B}$ are homotopic in the Lipschitzian category. 
It remains to show that $Y$ is $(\overline{\mathbf{H}}, 1)$ locally connected. Let $y_{0} \in Y=g_{r}(Q)$. If $y_{0} \notin B$ then $g_{r}^{-1}\left\{y_{0}\right\}=\left\{x_{0}\right\}$ is a singleton, $x_{0} \notin$ Clos $V_{r}$, and $g_{r}$ is a $C^{1}$ diffeomorphism near $x_{0}$ according to Theorem 12.3(A,C). Since $Q$ is $(\overline{\mathbf{H}}, 1)$ locally connected (Theorem 14.3(H”)) we conclude that $Y$ is $(\overline{\mathbf{H}}, 1)$ locally connected at $y_{0}$ (Lemma 8.4).

Assume now that $y_{0} \in B$ and let $U$ be an open set containing $y_{0}$. We seek an open set $U^{\prime} \subseteq U$ containing $y_{0}$ such that the homomorphisms induced by inclusion in homology, $\overline{\mathbf{H}}_{q}\left(Q \cap U^{\prime}\right) \rightarrow \overline{\mathbf{H}}_{q}(Q \cap U)$ are trivial for $q=0,1,2$. The cases $q=0,1$ on the one hand, and the case $q=2$ on the other hand, will follow from separate applications of Theorem 15.1 (after we define properly a family of sets $Y_{k, i}$ ). We now turn to defining the data that puts us in a position of applying Theorem 15.1.

We define $Q_{y_{0}}=Q \cap g_{r}^{-1}\left\{y_{0}\right\} \cap M_{r}\left(=Q \cap\left(y_{0}+W\left(y_{0}\right)\right) \cap M_{r} \cap \mathbf{B}(B, R)\right.$ with the notations of Theorem 12.3). Observe that $Q_{y_{0}}$ is finite. $\mathbb{P}$ Since $Q \cap \mathbf{U}\left(M_{r}, \delta\right)$ is 2dimensional polyhedral according to Theorem 14.3(M) there is a finite set $I$ and $x_{i} \in \mathbb{R}^{3}$, $W_{i} \in \mathbf{G}(3,2), i \in I$, such that $Q \cap \mathbf{U}\left(M_{r}, \delta\right) \subseteq \cup_{i \in I}\left(x_{i}+W_{i}\right) \cap \mathbf{U}\left(M_{r}, \delta\right)$. If $x \in Q_{y_{0}}$ then $x$ belongs to the intersection of the circle $M_{r} \cap\left(y_{0}+W\left(y_{0}\right)\right) \cap M_{r} \cap \mathbf{U}\left(y_{0}, R\right)$ and an affine line $\left(x_{i}+W_{i}\right) \cap\left(y_{0}+W\left(y_{0}\right)\right)$ for some $i \in I$ (notice that indeed $W_{i} \neq W\left(y_{0}\right)$ because $W\left(y_{0}\right)=W(\pi(x)) \notin \mathscr{W}_{Q}(x)$ as follows from Theorem 14.3(M3)). There are finitely many such points. Q We choose $r_{0}>0$ such that the balls $\mathbf{U}\left(x, r_{0}\right), x \in Q_{y_{0}}$, are pairwise disjoint and contained in $Q \cap g_{r}^{-1}(U)$. Next we put $\kappa=$ card $Q_{y_{0}}$, and we write $Q_{y_{0}}=\left\{x_{1}, \ldots, x_{\kappa}\right\}$. For each $k=1, \ldots, \kappa$ we let $\iota_{k}=\operatorname{card} \mathscr{W}_{Q}\left(x_{k}\right) \in\{1,2\}$ (Theorem 14.3(M1)). If $\iota_{k}=2$ we define $\bar{W}_{k}=\left(W_{1}\left(x_{k}\right) \cap W_{2}\left(x_{k}\right)\right)^{\perp}$ (where $\left\{W_{1}\left(x_{k}\right), W_{2}\left(x_{k}\right)\right\}=\mathscr{W}_{Q}\left(x_{k}\right)$ ), otherwise we define $\bar{W}_{k}=T_{x_{k}} M_{r}$. Notice that $\operatorname{dist}\left(\bar{W}_{k}, T_{x_{k}} M_{r}\right)<1$ (in the former case one refers to Theorem 14.3(M4)). Referring to Lemma 8.6 we see that for each $k=1, \ldots, \kappa$ there exists a "cylindrical" open set $O_{k} \subseteq \mathbf{U}\left(x_{k}, r_{0}\right)$ containing $x_{k}$, of the form $O_{k}=\mathbb{R}^{3} \cap\left\{\xi:\left|P_{\bar{W}_{k}}\left(\xi-x_{k}\right)\right|<r_{k}\right.$ and $\left.\left|P_{\bar{W}_{k}^{\perp}}\left(\xi-x_{k}\right)\right|<\delta_{k}\right\}$, and a Lipschitzian function $v_{k}:\left(x_{k}+\bar{W}_{k}\right) \cap O_{k} \rightarrow \bar{W}_{k}^{\perp}$ such that graph $v_{k}=M_{r} \cap O_{k}$. In view of Theorem 14.3(M2,M3) and Theorem 12.3(D) there is no restriction to assume that $O_{k}$ is sufficiently small for $g_{r} \uparrow\left(\left(x_{k}+W_{i}\left(x_{k}\right)\right) \cap O_{k} \backslash V_{r}\right)$ to be bi-Lipschitzian, $i=1, \ldots, \iota_{k}$. For $i=1, \ldots, \iota_{k}$ we also let $\Gamma_{k, i}^{0}=\left(x_{k}+W_{i}\left(x_{k}\right)\right) \cap M_{r} \cap O_{k}$.

We are now ready to define $Z$. Note that $g_{r}\left(\Gamma_{k, i}^{0}\right)$ are (for all the admissible indexes) neighborhoods of $y_{0}$ in $B$. Therefore their intersection contains an open connected neighborhood $Z$ of $y_{0}$ in $B$.

Next we turn to defining the sets $Y_{k, i}$ and the homotopies $H_{k, i}$. First we put $\Gamma_{k, i}=\left(g_{r} \uparrow\right.$ $\left.M_{r} \cap\left(x_{k}+W_{i}\left(x_{k}\right)\right) \cap O_{k}\right)^{-1}(Z)$ and $X_{k, i}=\left(x_{k}+W_{i}\left(x_{k}\right)\right) \cap O_{k} \cap P_{W_{k}}^{-1}\left(\Gamma_{k, i}\right) \backslash V_{r}, k=1, \ldots, \kappa$ and $i=1, \ldots, \iota_{k}$. Finally we put $Y_{k, i}=g_{r}\left(X_{k, i}\right)$. We leave to the reader the straightforward verification of hypotheses (B) and (C) of Theorem 15.1. Furthermore we define

$$
\bar{H}_{k}:[0,1] \times O_{k} \rightarrow O_{k}:(t, x) \mapsto\left(x+t\left(P_{x_{k}+\bar{W}_{k}}(x)+v_{k}\left(P_{x_{k}+\bar{W}_{k}}(x)\right)-x\right)\right)
$$

and we notice that $\bar{H}_{k}(t, x) \in X_{k, i}$ whenever $x \in X_{k, i}$. We also let

$$
H_{k, i}^{0}:[0,1] \times Y_{k, i} \rightarrow Y_{k, i}:(t, y) \mapsto g_{r}\left(\bar{H}_{k}\left(t,\left(g_{r}\left\lceil X_{k, i}\right)^{-1}(y)\right)\right) .\right.
$$

Observe that $\operatorname{Lip} H_{k, i}^{0}<\infty$ and $H_{k, i}^{0}(t, z)=z$ whenever $z \in Z$, for every $t \in[0,1]$ (this is because of the way $v_{k}$ is defined; indeed $x \in M_{r}$ implies $\bar{H}_{k}(t, x)=x$ for every $t \in[0,1]$, 
and if $z \in Z$ one has $\left(g_{r}\left\lceil X_{k, i}\right)^{-1}(z) \in \Gamma_{k, i} \subseteq M_{r}\right)$. We also consider a Lipschitzian map $H:[0,1] \times Z \rightarrow Z$ such that $H(0, z)=z$ and $H(1, z)=y_{0}$ for every $z \in Z$. We then define

$$
H_{k, i}:[0,1] \times Y_{k, i} \rightarrow Y_{k, i}:(t, y) \mapsto \begin{cases}H_{k, i}^{0}(2 t, y) & \text { if } 0 \leqslant t \leqslant 1 / 2 \\ H\left(2 t-1, H_{k, i}^{0}(1, y)\right) & \text { if } 1 / 2 \leqslant t \leqslant 1 .\end{cases}
$$

It is now an easy matter to check that hypotheses (D) and (E) of Theorem 15.1 are verified. On noticing that indeed $Y$ is a neighborhood of $y_{0}$ in $Q$ we infer the existence of an open set $U^{\prime} \subseteq \mathbb{R}^{3}$ such that $U^{\prime} \cap Q=Y$, thus

$$
\overline{\mathbf{H}}_{q}\left(Q \cap U^{\prime}\right) \rightarrow \overline{\mathbf{H}}_{q}(Q \cap U)
$$

are trivial for $q=0,1$.

Finally suppose that $T \in \mathbf{I}_{2}\left(Q \cap U^{\prime}\right)$ is so that $\partial T=0$. We aim to show that in fact $T=0$, so that $\overline{\mathbf{H}}_{2}\left(Q \cap U^{\prime}\right)=\{0\}$. Assume if possible that $T \neq 0$. Define

$$
Z^{*}=Z \cup\left(\cup_{\substack{k=1, \ldots, \kappa \\ \iota}=2} Y_{k, 1} \cap Y_{k, 2}\right) .
$$

Notice that $\mathscr{H}^{2}\left(Z^{*}\right)=0$, therefore $\operatorname{spt} T \nsubseteq Z^{*}$ (for otherwise $T=0$ ). Pick $y \in Y \cap(\operatorname{spt} T) \backslash$ $Z^{*}$. Choose $0<\eta<\operatorname{dist}(y, B)$ and define $T^{\prime}=T\left\llcorner\mathbb{R}^{3} \backslash \mathbf{B}(B, \eta)\right.$ and, in turn, $S^{\prime}=g_{r \#}^{-1} T^{\prime}$ (notice that $g_{r}^{-1}$ is a well-defined and bi-Lipschitzian homeomorphism on $\operatorname{spt} T^{\prime}$ ). Letting $\{x\}=g_{r}^{-1}\{y\}$ we infer that $x \in \operatorname{spt} S^{\prime}$. Now it follows from the constancy Theorem [11, 4.1.31(2)] that there exist $c \in \mathbb{R} \backslash\{0\}$ and $\xi \in \wedge_{2} \mathbb{R}^{3}$ orienting the plane that supports $X_{k, i}$ such that for every $x^{\prime} \in g_{r}^{-1}\left(Y_{k, i} \backslash\left(Z^{*} \cup \mathbf{B}(B, \eta)\right)\right)$ in the same component as $x$ there exists $\rho_{x^{\prime}}>0$ with $S^{\prime}\left\llcorner\mathbf{U}\left(x^{\prime}, \rho_{x^{\prime}}\right)=c \mathscr{H}^{2}\left\llcorner X_{k, i} \cap \mathbf{U}\left(x^{\prime}, \rho_{x^{\prime}}\right) \wedge \xi\right.\right.$. Choosing $x^{*} \in\left(x_{k}+W_{i}\left(x_{k}\right)\right) \cap$ Bdry $O_{k}$ in the same component as $x$ in $g_{r}^{-1}\left(Y_{k, i} \backslash\left(Z^{*} \cup \mathbf{B}(B, \eta)\right)\right)$ and observing that in a neighborhood of $x^{*}$ the support of $S^{\prime}$ is contained in $O_{k}$, we readily obtain $x^{*} \in \operatorname{spt} \partial S^{\prime}$, a contradiction.

\section{PART IV \\ MAIN RESULT}

\section{Toolkit}

We let $\check{H}(\cdot ; \mathbb{Z})$ denote the Čech homology functor with coefficients in $\mathbb{Z}$ defined on the category of compact pairs and their continuous maps (see [8, Chap. IX] and [5, 2.2]). The following is (essentially) taken from [23].

17.1. Proposition. - Assume that:

1. $B \subseteq \mathbb{R}^{3}$ is compact;

2. $S_{j} \subseteq \mathbb{R}^{3}, j=1,2, \ldots$, are compact and $B \subseteq S_{j}$ for every $j$;

3. $L \subseteq \check{H}_{1}(B ; \mathbb{Z})$ is a subgroup and $L \subseteq$ ker $\check{H}_{1}\left(i_{B, S_{j}}\right)$ for every $j=1,2, \ldots$ where $i_{B, S_{j}}$ denotes the inclusion map;

4. $S_{1}, S_{2}, \ldots$ converge in Hausdorff distance to some compact set $S \subseteq \mathbb{R}^{3}$.

Then $L \subseteq \operatorname{ker} \check{H}_{1}\left(i_{B, S}\right)$. 
Proof. - We define a decreasing sequence of compact sets $S_{j}^{\prime}=S \cup\left(\cup_{k=j}^{\infty} S_{k}\right), j=$ $1,2, \ldots$, and we notice that its inverse limit $S_{\infty}$ (see [8, Chap. VIII, Definition 3.1]) is homeomorphic to $S$. In fact letting $B_{\infty}$ denote the inverse limit of the inverse system " $B$ " (indexed by $j=1,2, \ldots$ and having each term constant equal to $B$ ) there are canonical homeomorphisms $f_{B}$ and $f_{S}$ such that the following diagram commutes (horizontal arrows denote inclusion)

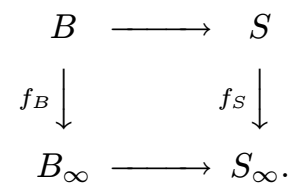

Observe that $L$ is contained in the algebraic boundary of $S_{j}^{\prime}$ because $S_{j} \subseteq S_{j}^{\prime}, j=1,2, \ldots$. We now consider the following commutative diagram.

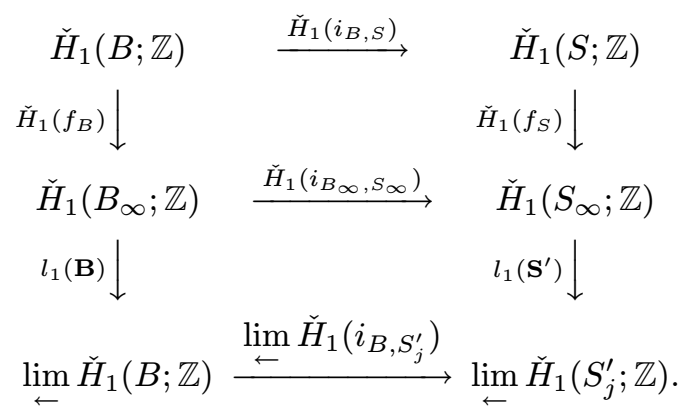

The vertical arrows in the first row are isomorphisms because $f_{B}$ and $f_{S}$ are homeomorphisms, whereas the vertical arrows of the second row are isomorphisms according to the continuity property of Čech homology with coefficients in $\mathbb{Z}$, [8, Chap. X, Theorem 3.1]. It follows from the definition of $\lim \check{H}_{1}\left(i_{B, S_{j}^{\prime}}\right), l_{1}(\mathbf{B})$ and $\check{H}_{1}\left(f_{B}\right)$ that the composition of these three homomorphisms maps any element of $L$ to 0 . The conclusion immediately follows.

Next we introduce the integral currents homology groups and a sufficient criterion for their coincidence with the Čech homology groups with coefficients in $\mathbb{Z}$. Given a set $X \subseteq \mathbb{R}^{3}$ we let $\mathbf{I}_{q}(X), q=0,1,2,3$, denote the group of integral currents $T \in \mathbf{I}_{q}\left(\mathbb{R}^{3}\right)$ such that spt $T \subseteq X$. The homology groups corresponding to the following chain complex are denoted by $\overline{\mathbf{H}}_{q}(X)$.

$$
\mathbf{I}_{3}(X) \stackrel{\partial}{\longrightarrow} \mathbf{I}_{2}(X) \stackrel{\partial}{\longrightarrow} \mathbf{I}_{1}(X) \stackrel{\partial}{\longrightarrow} \mathbf{I}_{0}(X) \stackrel{\alpha}{\longrightarrow} \mathbb{Z}
$$

(where $\alpha$ is an augmentation map defined by $\alpha(T)=\langle T, \mathbb{1}\rangle$ where $\mathbb{1}$ denotes any test function which equals 1 in a neighborhood of $\operatorname{spt} T$ ). A functor $\overline{\mathbf{H}}_{q}$ is then defined on the category of subsets of some Euclidean space and their locally Lipschitzian maps. It satisfies the axioms of Eilenberg-Steenrod. For details see [5] where the following result is proved as well.

17.2. Definition. - We say $X \subseteq \mathbb{R}^{3}$ is $(\overline{\mathbf{H}}, 1)$ locally connected if the following holds. For every $x \in X$ and every open set $U \subseteq \mathbb{R}^{3}$ containing $x$ there exists an open set $U^{\prime} \subseteq U$ containing $x$ such that the homomorphisms induced in homology by inclusion are trivial, $\overline{\mathbf{H}}_{q}\left(X \cap U^{\prime}\right) \rightarrow \overline{\mathbf{H}}_{q}(X \cap U), q=0,1,2$. 
17.3. Proposition. - Let $\mathscr{A}^{L C, \overline{\mathbf{H}}, 1}$ denote the category whose objects are the $(\overline{\mathbf{H}}, 1)$ locally connected subsets of some Euclidean space $\mathbb{R}^{n}$, together with their locally Lipschitzian maps, and let $\operatorname{Mod}_{\mathbb{Z}}$ denote the category of modules over $\mathbb{Z}$. The functors

$$
\overline{\mathbf{H}}_{1}: \mathscr{A}^{L C, \overline{\mathbf{H}}, 1} \longrightarrow \operatorname{Mod}_{\mathbb{Z}} \quad \text { (integral currents homology) }
$$

and

$$
\check{H}_{1}(\cdot ; \mathbb{Z}): \mathscr{A}^{L C, \overline{\mathbf{H}}, 1} \longrightarrow \operatorname{Mod}_{\mathbb{Z}} \quad \text { ( } \check{C} \text { ech homology with coefficients in } \mathbb{Z} \text { ) }
$$

are naturally equivalent.

In the remainder of this paper we will denote by $\nu: \overline{\mathbf{H}}_{1} \rightarrow \check{H}_{1}(\cdot ; \mathbb{Z})$ the natural equivalence whose existence is asserted above.

17.4. Remark. - With the vocabulary at hand it is now easy to relate $B_{0}$ and $L$ rigorously. Indeed we let $L_{0}$ be the subgroup of $\overline{\mathbf{H}}_{1}(B)$ generated by the class $\left[B_{0}\right]$, and $L=$ $\nu_{B}\left(L_{0}\right)$. This is well-defined since $B$ is $(\overline{\mathbf{H}}, 1)$ locally connected.

The following is nearly trivial and very useful. It justifies the introduction of the $(\overline{\mathbf{H}}, 1)$ locally connected spaces.

17.5. Proposition. - Assume that $S \subseteq \mathbb{R}^{3}$ contains $B$ and is $(\overline{\mathbf{H}}, 1)$ locally connected. The following conditions are equivalent.

(A) $L$ is contained in the algebraic boundary of $S$;

(B) There exists $T \in \mathscr{R}_{2}\left(\mathbb{R}^{3}\right)$ such that $\operatorname{spt} T \subseteq S$ and $\partial T=B_{0}$.

Proof. - Since both $B$ and $S$ are objects of the category $\mathscr{A}^{L C, \overline{\mathbf{H}}, 1}$, Proposition $17.3 \mathrm{im}$ plies the existence of the following commutative diagram whose vertical arrows are isomorphisms.

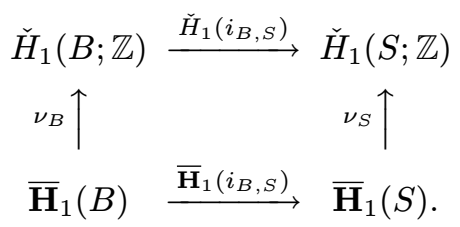

Assuming (A) holds we infer that $\overline{\mathbf{H}}_{1}\left(i_{B, S}\right)\left(\left[B_{0}\right]\right)=\left[i_{B, S \#} B_{0}\right]=\left[B_{0}\right]$ vanishes in $\overline{\mathbf{H}}_{1}(S)$. The definition of $\overline{\mathbf{H}}_{1}(S)$ then readily implies (B). That (B) implies (A) follows from the same observation.

In view of the previous result the approximation theorem 6.1 is useful for replacing any set with a set of nearly the same size and verifying the required boundary condition in both settings. When applying Theorem 6.1 the following will be handy.

17.6. Proposition. - Let $f: \mathbb{R}^{3} \rightarrow \mathbb{R}^{3}$ be a Lipschitzian map such that $f(B)=B$ and $f \uparrow B$ is homotopic to the identity of $B$ in the Lipschitzian category. The following hold.

(A) If the algebraic boundary of some set $S \supseteq B$ contains $L$, then so does the algebraic boundary of $f(S)$;

(B) If $T \in \mathscr{R}_{2}\left(\mathbb{R}^{3}\right)$ and $\partial T=B_{0}$ then $\partial f_{\#} T=B_{0}$. 
Proof. - Conclusion (A) follows from the fact that $\check{H}_{1}(f) \circ \check{H}_{1}\left(i_{B, S}\right)=\check{H}_{1}\left(f \circ i_{B, S}\right)=$ $\check{H}_{1}\left(i_{B, f(S)}\right)$, the last equality being a consequence of the Homotopy Axiom and the fact that $f \circ i_{B, S}$ and $i_{B, f(S)}$ are homotopic. In order to prove conclusion (B) we consider a Lipschitzian map $F:[0,1] \times B \rightarrow B$ such that $F(0, x)=x$ and $F(1, x)=f(x)$ for every $x \in B$, and we extend it to a Lipschitzian map $\hat{F}:[0,1] \times \mathbb{R}^{3} \rightarrow \mathbb{R}^{3}$. The homotopy formula for integral currents yields

$$
\partial f_{\#} T-\partial T=f_{\#} \partial T-\partial T=\partial \hat{F}_{\#}(\llbracket 0,1 \rrbracket \times \partial T)=0 .
$$

The latter is a consequence of the fact that $\hat{F}_{\#}(\llbracket 0,1 \rrbracket \times \partial T) \in \mathscr{R}_{2}\left(\mathbb{R}^{3}\right)$ together with the inequality

$$
\begin{aligned}
\mathscr{H}^{2}\left(\operatorname{spt} \hat{F}_{\#}(\llbracket 0,1 \rrbracket \times \partial T)\right) & \leqslant \mathscr{H}^{2}(\hat{F}([0,1] \times \operatorname{spt} \partial T)) \\
& \leqslant \mathscr{H}^{2}(F([0,1] \times B))=\mathscr{H}^{2}(B)=0 .
\end{aligned}
$$

For a rectifiable current $T \in \mathscr{R}_{2}\left(\mathbb{R}^{3}\right)$ we let $\|T\|$ denote the measure $|\theta| \mathscr{H}^{2}\llcorner M$ (see also $[11,4.1 .7])$. For a Radon measure $\phi$ in an open set $U \subseteq \mathbb{R}^{3}$ we define

$$
\Theta^{2}(\phi, x)=\lim _{r \downarrow 0} \frac{\phi(\mathbf{B}(x, r))}{\pi r^{2}}
$$

$x \in U$ (whenever the limit exists), and

$$
\operatorname{set}_{2}(\phi)=U \cap\left\{x: 0<\Theta^{2}(\phi, x)<\infty\right\} .
$$

Size can then be defined as $\mathbf{S}(T)=\mathscr{H}^{2}\left(\operatorname{set}_{2}(\|T\|)\right), T \in \mathscr{R}_{2}\left(\mathbb{R}^{3}\right)$. It is lower semicontinuous with respect to integral flat convergence (see for instance [7, Lemma 3.2.14]). Thus for every $\varepsilon>0$ the compactness Theorem 3.1 implies that the following variational problem admits a minimizer.

$$
\left(\mathscr{P}_{F F, \mathbf{S}, B_{0}}^{\varepsilon}\right)\left\{\begin{array}{l}
\text { minimize } \mathbf{S}(T)+\varepsilon \mathbf{M}(T) \\
\text { among } T \in \mathscr{R}_{2}\left(\mathbb{R}^{3}\right) \text { such that } \partial T=B_{0} .
\end{array}\right.
$$

Simple considerations in [7, Lemma 2.1.1] show that

$$
\lim _{\varepsilon \rightarrow 0} \inf \left(\mathscr{P}_{F F, \mathbf{S}, B_{0}}^{\varepsilon}\right)=\inf \left(\mathscr{P}_{F F, \mathbf{S}, B_{0}}\right) .
$$

The support of a minimizing current relative to the problem above enjoys some weak regularity property called monotonicity which we now describe.

17.7. Definition. - A Radon measure $\phi$ defined in an open set $U \subseteq \mathbb{R}^{3}$ is called 2-monotonic whenever the following holds. For every $x \in U$ the function

$$
(0, \operatorname{dist}(x, \operatorname{Bdry} U)) \rightarrow \mathbb{R}: r \mapsto \frac{\phi(\mathbf{B}(x, r))}{\pi r^{2}}
$$

is nondecreasing. If also $\Theta^{2}(\phi, x) \geqslant 1$ for every $x \in \operatorname{spt} \phi$ then we say that $\phi$ is 2 -concentrated.

The following can be proved for instance as in [6, Proposition 3.4.5].

17.8. Proposition. - If $T \in \mathscr{R}_{2}\left(\mathbb{R}^{3}\right)$ is a minimizing current relative to $\left(\mathscr{P}_{F F, \mathbf{S}, B_{0}}^{\varepsilon}\right)$ then the measure

$$
\phi=\left(1+\varepsilon \Theta^{2}(\|T\|, \cdot)\right) \mathscr{H}^{2}\left\llcorner\operatorname{set}_{2}(\|T\|)\right.
$$

is 2-monotonic and 2-concentrated in $U=\mathbb{R}^{3} \backslash B$.

$4^{\text {e }}$ SÉRIE - TOME $42-2009-\mathrm{N}^{\mathrm{o}} 1$ 
We will need two properties related to monotonicity. For a proof, see [4, Proposition 4.3 and Corollary 6.13].

17.9. Proposition. - Let $\phi_{1}, \phi_{2}, \ldots$ be a sequence of 2-concentrated 2-monotonic measures in an open set $U \subseteq \mathbb{R}^{3}$ and assume that $\sup _{j} \phi_{j}(U)<\infty$. There then exist a subsequence $\phi_{j(1)}, \phi_{j(2)}, \ldots$ and a Radon measure $\phi$ in $U$ such that

(A) $\phi_{j(k)} \rightarrow \phi$ weakly* as $k \rightarrow \infty$;

(B) $\phi$ is 2-concentrated and 2-monotonic;

(C) For every compact $C \subseteq U$,

$$
\begin{aligned}
& \lim _{k \rightarrow \infty} \inf \left\{\delta>0: \operatorname{spt}(\phi) \cap C \subseteq \mathbf{B}\left(\operatorname{spt}\left(\phi_{j(k)}\right), \delta\right)\right. \\
& \left.\quad \text { and } \operatorname{spt}\left(\phi_{j(k)}\right) \cap C \subseteq \mathbf{B}(\operatorname{spt}(\phi), \delta)\right\}=0 .
\end{aligned}
$$

17.10. Proposition. - Let $\phi$ be a 2-concentrated 2-monotonic measure in an open set $U \subseteq \mathbb{R}^{3}$ with $\phi(U)<\infty$. Then $\operatorname{spt} \phi=\operatorname{set}_{2}(\phi)$ is $\left(\mathscr{H}^{2}, 2\right)$ rectifiable.

\section{Existence theorem}

We are now in a position to give the proof of Theorem 5.1. Before starting we notice that the collection of competitors is nonempty (for both formulations of the problem) as follows from a cone construction.

First we show that

$$
\inf \left(\mathscr{P}_{F F, \mathbf{S}, B_{0}}\right) \leqslant \inf \left(\mathscr{P}_{R, \mathbb{Z}, L}\right) .
$$

Let $X$ be a competitor for $\left(\mathscr{P}_{R, \mathbb{Z}, L}\right)$ and $\varepsilon>0$. Let $Y$ be associated with $X$ and $\varepsilon$ in Theorem 6.1. It follows from Theorem 6.1(I) together with Proposition 17.6 that the algebraic boundary of $Y$ contains $L$, and in turn we infer from Theorem 6.1(H) and Proposition 17.5 that there exists $T \in \mathscr{R}_{2}\left(\mathbb{R}^{3}\right)$ with $\partial T=B_{0}$ and $\operatorname{spt} T \subseteq Y$. Since

$$
\mathbf{S}(T)=\mathscr{H}^{2}\left(\operatorname{set}_{2}(\|T\|)\right) \leqslant \mathscr{H}^{2}(\operatorname{spt} T) \leqslant \mathscr{H}^{2}(Y) \leqslant \varepsilon+\mathscr{H}^{2}(X),
$$

inequality (75) follows from the arbitrariness of $X$ and $\varepsilon$.

Next we choose $\varepsilon_{j} \downarrow 0$ as $j \rightarrow \infty$ and we let $T_{j}, j=1,2, \ldots$, denote a minimizer of $\left(\mathscr{P}_{F F, \mathbf{S}, B_{0}}^{\varepsilon_{j}}\right)$. We denote by $\phi_{j}, j=1,2, \ldots$, the 2-monotonic 2-concentrated measures associated with $T_{j}$ in Proposition 17.8. Passing to a subsequence (still denoted by $\phi_{j}$ ) if necessary we may assume that the conclusions of Proposition 17.9 are satisfied. Letting $X_{j}=$ $\operatorname{spt}\left(T_{j}\right)=\operatorname{spt}\left(\phi_{j}\right) \cup B$ we infer from Proposition 17.9(C) that $\operatorname{dist}_{\mathscr{H}}\left(X_{j}, \operatorname{spt}(\phi) \cup B\right) \rightarrow 0$ as $j \rightarrow \infty$ (Hausdorff distance). Next we let $Y_{j}$ and $f_{j}$ be associated with $X_{j}$ and $\varepsilon_{j}$ in Theorem 6.1. Since $f_{j} \uparrow B$ is homotopic to the identity of $B$ in the Lipschitzian category we infer from Proposition 17.6(B) that $\partial f_{j \#} T_{j}=B_{0}$, and since spt $f_{j \#} T_{j} \subseteq f_{j}\left(\operatorname{spt} T_{j}\right) \subseteq Y_{j}$ (according to Theorem 6.1(D)) we infer from Proposition 17.5 that the algebraic boundary of Clos $Y_{j}$ contains $L$. Theorem 6.1(E) says that $\operatorname{dist}_{\mathscr{H}}\left(X_{j}, \operatorname{Clos} Y_{j}\right)<\varepsilon_{j}$. Therefore $\operatorname{dist}_{\mathscr{H}}\left(\operatorname{Clos} Y_{j}, \operatorname{spt}(\phi) \cup B\right) \rightarrow 0$ as $j \rightarrow \infty$. Now Proposition 17.1 implies that the algebraic 
boundary of $S=\operatorname{spt}(\phi) \cup B$ contains $L$. Furthermore $S$ is $\left(\mathscr{H}^{2}, 2\right)$ rectifiable according to Proposition 17.10 so that it is an admissible competitor for $\left(\mathscr{P}_{R, \mathbb{Z}, L}\right)$. Finally,

$$
\begin{aligned}
\mathscr{H}^{2}(S) & =\mathscr{H}^{2}(\operatorname{spt}(\phi)) \quad \quad \text { (because } \phi \text { is 2-concentrated) } \\
& \leqslant \phi(U) \quad \\
& =\lim _{j} \phi_{j}(U) \quad \\
& =\lim _{j} \inf \left(\mathscr{P}_{F F, \mathbf{S}, B_{0}}^{\varepsilon_{j}}\right) \\
& =\inf \left(\mathscr{P}_{F F, \mathbf{S}, B_{0}}\right),
\end{aligned}
$$

and both conclusions are proved at once.

\section{REFERENCES}

[1] W. K. Allard, On the first variation of a varifold: boundary behavior, Ann. of Math. 101 (1975), 418-446.

[2] K. A. Brakke, The surface evolver, Experiment. Math. 1 (1992), 141-165.

[3] R. Courant, Dirichlet's Principle, Conformal Mapping, and Minimal Surfaces, Interscience Publishers, Inc., 1950.

[4] T. De Pauw, Nearly flat almost monotone measures are big pieces of Lipschitz graphs, J. Geom. Anal. 12 (2002), 29-61.

[5] T. De PAUw, Comparing homologies: Čech's theory, singular chains, integral flat chains and integral currents, Rev. Mat. Iberoam. 23 (2007), 143-189.

[6] T. De Pauw, Concentrated, nearly monotonic, epiperimetric measures in Euclidean space, J. Differential Geom. 77 (2007), 77-134.

[7] T. De Pauw, R. Hardt, Size minimization and approximating problems, Calc. Var. Partial Differential Equations 17 (2003), 405-442.

[8] S. Eilenberg, N. Steenrod, Foundations of algebraic topology, Princeton University Press, 1952.

[9] L. C. Evans, R. F. Gariepy, Measure theory and fine properties of functions, Studies in Advanced Mathematics, CRC Press, 1992.

[10] H. Federer, Curvature measures, Trans. Amer. Math. Soc. 93 (1959), 418-491.

[11] H. Federer, Geometric measure theory, Die Grund. Math. Wiss., Band 153, Springer, 1969.

[12] H. Federer, W. H. Fleming, Normal and integral currents, Ann. of Math. 72 (1960), 458-520.

[13] V. Feuvrier, Un résultat d'existence pour les ensembles minimaux par optimisation sur des grilles polyédrales, Thèse de doctorat, Université d'Orsay, 2008.

[14] W. H. Fleming, An example in the problem of least area, Proc. Amer. Math. Soc. 7 (1956), 1063-1074.

[15] W. H. Fleming, On the oriented Plateau problem, Rend. Circ. Mat. Palermo 11 (1962), 69-90.

$4^{\mathrm{e}}$ SÉRIE - TOME $42-2009-\mathrm{N}^{\mathrm{o}} 1$ 
[16] R. L. Foote, Regularity of the distance function, Proc. Amer. Math. Soc. 92 (1984), $153-155$.

[17] E. Lamarle, Sur la stabilité des systèmes liquides en lames minces, Mémoires de l'Académie Royale de Belgique 35 (1864).

[18] G. Lawlor, F. Morgan, Curvy slicing proves that triple junctions locally minimize area, J. Differential Geom. 44 (1996), 514-528.

[19] F. Morgan, Size-minimizing rectifiable currents, Invent. Math. 96 (1989), 333-348.

[20] F. Morgan, Geometric measure theory, a beginner's guide, third ed., Academic Press Inc., 2000.

[21] D. Pavlica, personal communication.

[22] J. Plateau, Statique expérimentale et théorique des liquides soumis aux seules forces moléculaires, Gauthier-Villars, 1873.

[23] E. R. Reifenberg, Solution of the Plateau Problem for $m$-dimensional surfaces of varying topological type, Acta Math. 104 (1960), 1-92.

[24] E. R. Reifenberg, An epiperimetric inequality related to the analyticity of minimal surfaces, Ann. of Math. 80 (1964), 1-14.

[25] G. DE R ham, Variétés différentiables. Formes, courants, formes harmoniques, Hermann, 1973.

[26] L. Simon, Lectures on geometric measure theory, Proceedings of the Centre for Mathematical Analysis, Australian National University, 1983.

[27] J. E. TAYLOR, The structure of singularities in soap-bubble-like and soap-film-like minimal surfaces, Ann. of Math. 103 (1976), 489-539.

[28] H. Whitney, Geometric integration theory, Princeton University Press, 1957.

(Manuscrit reçu le 23 novembre 2007; accepté, après révision, le 3 juillet 2008.)

Thierry De PAuw

Université catholique de Louvain

Département de Mathématiques

Chemin du cyclotron, 2

1348 Louvain-la-Neuve, Belgique

E-mail: thierry.depauw@uclouvain.be 